Portland State University

PDXScholar

Fall 12-4-2013

\title{
A Theory of Travel Decision-Making with Applications for Modeling Active Travel Demand
}

\author{
Patrick Allen Singleton \\ Portland State University
}

Follow this and additional works at: https://pdxscholar.library.pdx.edu/open_access_etds

Part of the Transportation Commons

Let us know how access to this document benefits you.

\section{Recommended Citation}

Singleton, Patrick Allen, "A Theory of Travel Decision-Making with Applications for Modeling Active Travel Demand" (2013). Dissertations and Theses. Paper 1494.

https://doi.org/10.15760/etd.1493

This Thesis is brought to you for free and open access. It has been accepted for inclusion in Dissertations and Theses by an authorized administrator of PDXScholar. Please contact us if we can make this document more accessible: pdxscholar@pdx.edu. 
A Theory of Travel Decision-Making with

Applications for Modeling Active Travel Demand

by

Patrick Allen Singleton

A thesis submitted in partial fulfillment of the requirements of the degree of

Master of Science

in

Civil and Environmental Engineering

Thesis Committee:

Kelly J. Clifton, Chair

Christopher M. Monsere

Liming Wang

Portland State University

2013 
C 2013 Patrick Allen Singleton 


\section{ABSTRACT}

The continuing evolution of urban travel patterns and changing policy goals and priorities requires that transportation researchers and practitioners improve their abilities to plan and forecast the demand for travel. Walking and bicycling - the primary forms of active travel - are generating increasing interest for their potential to reduce automobile use, save governmental and consumer costs, and improve personal and social health outcomes. Yet, current transportation planning tools, namely regional travel demand forecasting models, poorly represent these active travel modes, if at all.

More broadly, travel models do an incomplete job of representing the decisionmaking processes involved in travel choices, especially those factors influencing walking and bicycling. In addition to limitations of data and statistical analysis methods, the research upon which modeling tools are based has yet to settle on a comprehensive theory of travel behavior that accounts for complex relationships around a variety of personal, social, and environmental factors. While modeling tools have explained travel primarily through economic theories, contributions from the geography and psychology fields prove promising. A few scholars have attempted to link these travel behavior explanations together, some with a focus on walking and bicycling, but these theories have yet to make a significant impact on travel modeling practice.

This thesis presents a unifying interdisciplinary framework for a theory of travel decision-making with applications for travel demand modeling and forecasting and a focus on walking and bicycling. The framework offers a guide for future research examining the complex relationships of activities, built environment factors, 
demographic and socioeconomic characteristics, attitudes and perceptions, and habit and exploration on individual short-term travel decisions (with considerations of the influence of medium- and long-term travel-related decisions). A key component of the theory is a hierarchy of travel needs hypothesized to be considered by travelers in the course of their decision-making processes. Although developed to account for the factors that particularly influence decisions surrounding walking and bicycling, the framework is postulated to apply to all travel modes and decisions, including frequency, destination, mode, time-of-day, and route.

The first section of the thesis reviews theories from the fields of economics, geography, psychology, and travel behavior that have a large influence on the development of the theory of travel decision-making. In the next and largest chapter, the components and relationships in this theory, including the hierarchy of travel needs, are defined and presented with supporting empirical evidence from travel behavior research.

This thesis's final section views the theory of travel decision-making through the lens of applicability to travel demand modeling and forecasting. The state of current travel forecasting tools, travel behavior research, data, and analysis methods with respect to each aspect of the theory is reviewed. Research and data needs are identified. In closing, some opportunities for operationalizing the theory in travel demand models and using these transportation planning tools for analyzing walking, cycling, and other policies are hypothesized and discussed. This thesis, and the theory and applications discussed within, contribute to the academic study of travel behavior, the practical modeling of travel demand, and walking and bicycling research and planning. 


\section{DEDICATION}

To future generations of active travelers - be they walking, cycling, skating, skipping, scooting, rolling, or otherwise moving under human power - in the hopes that one day the transportation planning and engineering professions will give you the same dignity and attention as is given to other road users. 


\section{ACKNOWLEDGEMENTS}

I share this accomplishment with many people and organizations deserving of thanks. The U.S. Department of Transportation provided generous financial support in the form of graduate fellowships through the Dwight David Eisenhower Transportation Fellowship Program. Portland State University (PSU), the Oregon Transportation Research and Education Consortium (OTREC), and Dick Walker of Metro offered additional financial and professional support and facilitated a research project that started me down this path.

Other graduate students at PSU, especially the PhT group (PhD Students in Transportation), helped me solve some of the challenges involved with developing the theory and provided a strong sense of camaraderie the last two years. Members of my committee, Dr. Chris Monsere and Dr. Liming Wang, deserve great credit for providing constructive criticism and suggestions as well as excellent learning environments in their classes. I am obliged to Dr. Robert Schneider, honorary committee member, for several times offering his critical eye and valuable discussions of travel behavior theory. Many thanks are also due to my advisor, Dr. Kelly Clifton, who - more than anyone else guided and motivated my interest in travel behavior and encouraged an engineering student to write a theoretical thesis.

Finally, I deeply thank my friends, family, and partner Jen for their love, support, interest, and tolerance of many nights and weekends spent reading and typing. I could not have done this without all of you. 


\section{TABLE OF CONTENTS}

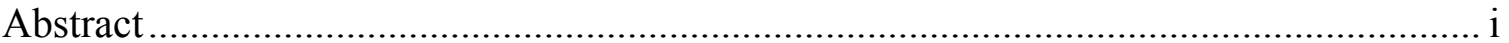

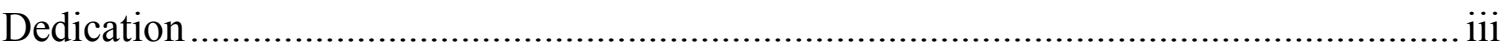

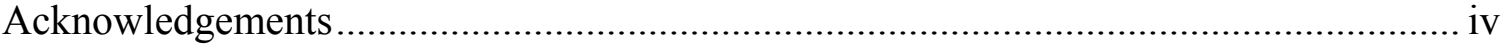

List of Tables .................................................................................................... vii

List of Figures ............................................................................................. viii

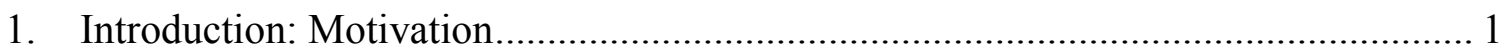

2. Background: Theories for Travel Behavior............................................................ 4

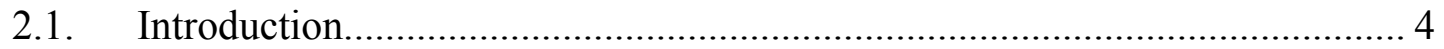

2.2. Economic Theories ............................................................................... 6

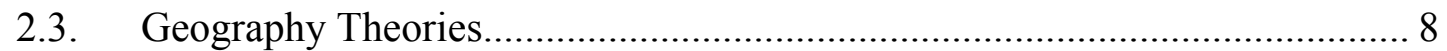

2.4. Psychological Behavior Theories .......................................................... 12

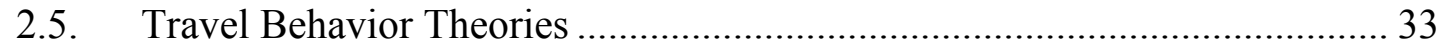

2.6. Conclusion .................................................................................. 41

3. Theory of Travel Decision-Making: A Unifying Framework ............................... 43

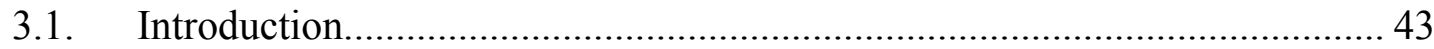

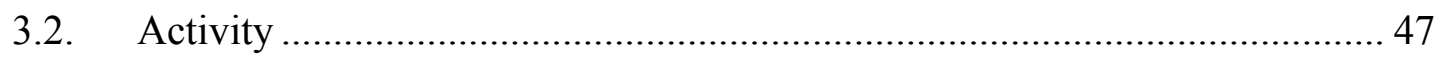

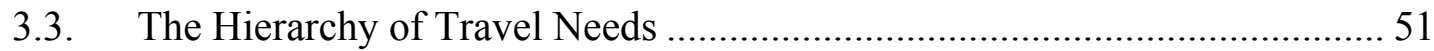

3.4. Moderation and Indirect Effects: Demographics and Socioeconomics....... 105

3.5. Mediation: The Perception Lens.......................................................... 112

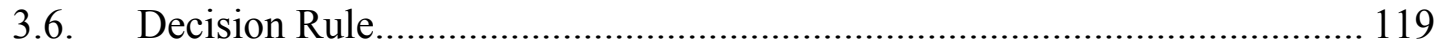

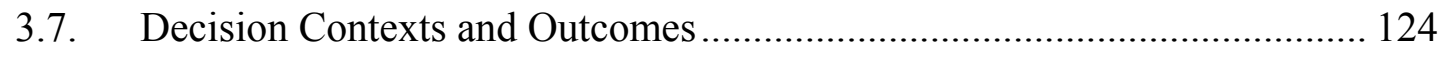

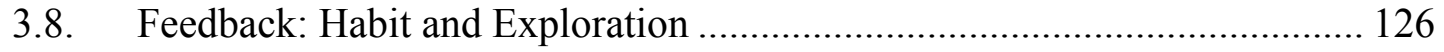




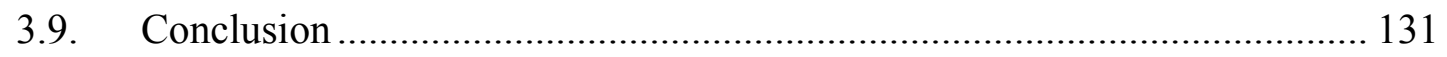

4. Applying the Theory to Travel Demand Forecasting Models ............................... 135

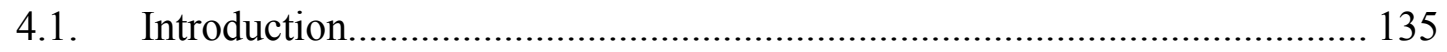

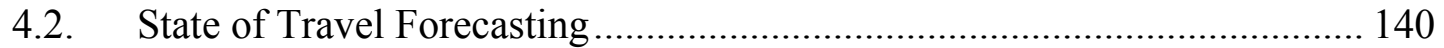

4.3. State of Travel Behavior Research ...................................................... 158

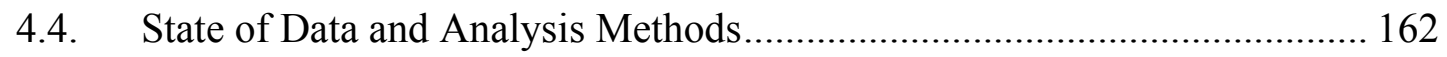

4.5. Opportunities for Operationalization and Use in Travel Forecasting.......... 177

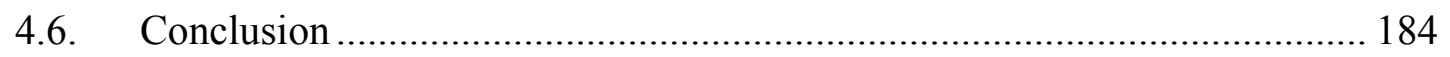

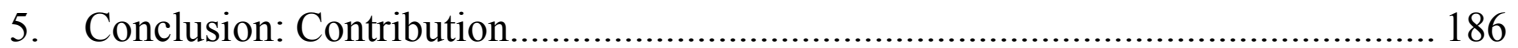

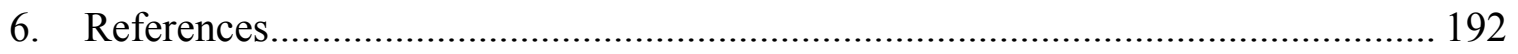




\section{LIST OF TABLES}

Table 4-1: State of the Theory of Travel Decision-Making with Respect to Travel Forecasting and Modeling, Travel Behavior Research, and Data and Analysis Methods 


\section{LIST OF FIGURES}

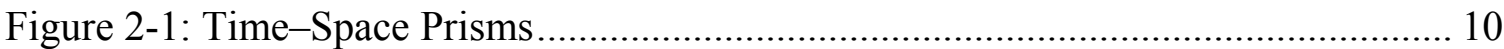

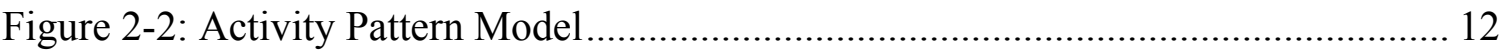

Figure 2-3: Hierarchy of Needs for Human Motivation ................................................. 16

Figure 2-4: Theory of Reasoned Action and Theory of Planned Behavior ...................... 19

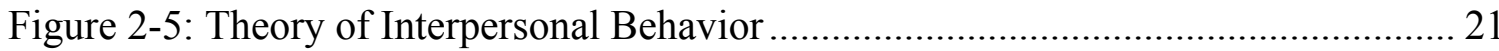

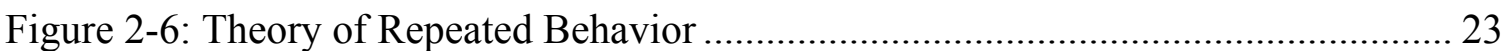

Figure 2-7: Reciprocal Determinism in Social Cognitive Theory ……............................. 25

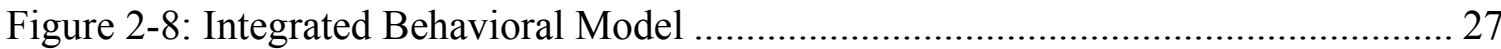

Figure 2-9: Normative Decision-Making Model …………............................................ 32

Figure 2-10: Comprehensive Action Determination Model .............................................. 33

Figure 2-11: Social-Ecological Framework for Walking …………………………........ 35

Figure 2-12: Theory of Routine Mode Choice Decisions................................................ 39

Figure 3-1: Conceptual Framework of the Theory of Travel Decision-Making ............... 44

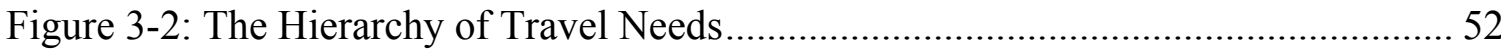

Figure 4-1: Active Travel Modeling Frameworks ...................................................... 144

Figure 4-2: Trip-Based Models in the Theory of Travel Decision-Making ................... 147

Figure 4-3: Activity-Based Models in the Theory of Travel Decision-Making ............. 147 


\section{INTRODUCTION: MOTIVATION}

All truly great thoughts are conceived by walking. (attributed to Friedrich Nietzsche)

The continuing evolution of urban travel patterns and changing transportation policy goals and priorities require that transportation researchers and practitioners improve their abilities to plan and forecast the demand for travel. In an era of constrained resources and competing interests, greater requirements are being placed upon the travel demand forecasting models used for metropolitan area planning. These tools are increasingly being asked to address policy concerns beyond the strict estimation of traffic flows, including aspects of multimodal performance measures, tolling, land use development, air quality, transportation emissions, climate change, energy and environmental sustainability, equity, public heath, and non-motorized modes.

Walking and bicycling - the primary forms of active travel - are generating increasing interest for their potential to reduce automobile use, save governmental and consumer costs, and improve personal and social health outcomes. In response to recent initiatives in the US and elsewhere (e.g., Safe Routes to School, Complete Streets, Active Living, Green Lane Project), there is a desire to better plan and model the increasing demand for walking and cycling. Better representation of these modes in travel demand models improves the tools' sensitivities to predicting the mode shift effects of economic changes and interventions, addressing many current policy concerns. Legislation, from the federal Clean Air Act Amendments of 1990 and the Intermodal Surface 
Transportation Efficiency Act of 1991 to recent state laws regarding climate change (California Senate Bill 375 and Oregon Senate Bill 1059), has mandated changes to transportation planning processes to be more inclusive of walking and bicycling influences and impacts. Yet, current transportation planning tools, namely regional travel demand forecasting models, poorly represent these active travel modes, if at all.

More broadly, these travel models do a poor job of representing the decisionmaking processes involved in travel choices, especially of those factors influencing walking and bicycling. In addition to limitations of data and statistical analysis methods, a reason for this state may be that the travel behavior research upon which modeling tools are based has yet to settle on a comprehensive theory of travel behavior that accounts for complex relationships around a variety of personal, social, and environmental factors. Environmental factors in particular are thought to affect walking and bicycling in much different ways than other travel modes. While modeling tools have explained travel primarily through microeconomic theories of utility maximization, theoretical contributions from the transport geography and social/environmental psychology fields prove promising. A few scholars have attempted to link these travel behavior explanations together, some with a focus on walking and bicycling, including the theory of routine mode choice decisions (Schneider, 2013) and the hierarchy of walking needs (Alfonzo, 2005). Although these theories are helpful for structuring future research on active travel behavior, they have yet to make a significant impact on travel modeling practice. A conceptual framework including walking and bicycling behavioral relationships from multiple disciplines yet structured with travel forecasting applications in mind could make contributions to both research and practice. 
This thesis presents such a unifying interdisciplinary framework of travel behavior. Specifically, it develops a theory of travel decision-making with several major contributions. First, the theory informs academic research and future theories of travel behavior. By integrating travel behavior knowledge with research from the fields of economics, geography, and psychology, the thesis develops a conceptual framework of travel behavior that accounts for the complex relationships among personal, social, and environmental factors. Second, the theory is directly applicable to the practice of travel demand forecasting. The relationships are structured with modeling applications in mind, suggesting means of operationalizing components into working travel models and yielding more behaviorally-realistic travel forecasting tools. Finally, both contributions combine to yield advances in walking and bicycling research and planning. The theory is explicitly designed to represent factors of importance to potential active travelers, and it suggests one way in which travel demand models can account for these influences.

In summary, this thesis develops and discusses a unifying theory of travel decision-making with applications for travel modeling and forecasting and a focus on walking and bicycling. Chapter 2 provides a theoretical background by introducing a number of theories to explain (active) travel behavior from the fields of economics, geography, and psychology. Chapter 3 defines and describes each component and relationship within the conceptual framework alongside supporting theoretical and empirical evidence. Chapter 4 discusses applications of the theory to travel demand modeling, including the state of travel forecasting, travel behavior research, data, and analysis methods with respect to each aspect of the theory. The thesis closes with an analysis of future opportunities and a summarizing chapter. 


\section{BACKGROUND: THEORIES FOR TRAVEL BEHAVIOR}

Theory provides the basis for conceptual models, consisting of the behavior of interest and the factors that explain that behavior, the ways in which these variables are defined, and the assumed relationships between them, that researchers use as an essential guide to their efforts. (Handy, 2005, p. 1)

\subsection{Introduction}

A theory is a valuable tool in the scientific study of phenomena, especially when related to human behavior - which includes travel behavior. Although specific scientific fields may have different customs and conventions on how theories are used in research development and data analysis, theory plays an important role in both theory-driven fields and empirically-focused work. Theories often describe a conceptual model of behavior, which prescribes the behavior of interest, conceptual factors that may influence or explain the behavior, and hypothetical relationships between them (Handy, 2005). These assumed factors and relationships are often translated into operational variables and mathematical relationships which make up a scientific hypothesis that can be tested. Even in purely empirical study, some sort of theoretical background is employed to determine what kinds of data are to be collected. Hypothesis testing is an important part of behavioral analysis because it can be used to support an existing theory, challenge an existing theory, or create a new theory of behavior. 
Travel behavior research, to the extent that it is theoretically-grounded, utilizes a number of theories from various scientific fields. Theories from the fields of economics, geography, and psychology are particularly useful in providing explanations for many travel and transportation-related choices and observations. Historically, the development of travel demand forecasting models used in transportation planning practice has relied less on theories than on basic mathematical relationships designed to best fit aggregate data summarizing the behavior of groups of people. Although the recent focus on explaining travel demand using models of individual behavior has facilitated a more rigorous inclusion of theories from these three main fields, consideration of the factors and relationships relevant to walking and bicycling has been less theoretically methodical. A primary goal of this research is to change this trend and introduce empirically-testable and theoretically-based hypotheses into the practice of forecasting active travel.

The sections of this chapter describe some of the theories from the fields of economics, geography, and psychology that have influenced the study of travel behavior and the forecasting of travel demand. The key contributions from each theory, their relevance to the development of the theory of travel decision-making presented in this thesis, and their possible applications to the forecasting of walking and bicycling are described and noted. In addition, two key theories arising out of travel behavior literature that focus on explaining active travel are presented: Alfonzo's hierarchy of walking needs (2005) and Schneider's theory of routine mode choice decisions (2013). These two theories, more than any others, provide the basis for the theory of travel decision-making 
- described in a later section - and its application to the modeling of active travel behavior and demand.

\subsection{Economic Theories}

The field of economics has contributed heavily to the development of theories, analysis methods, and tools to examine travel behavior, particularly in the realm of specifying, estimating, and applying travel demand forecasting models. The most common econometric theory used in the transportation realm is the theory of random utility maximization; Daniel McFadden shared the 2000 Nobel Prize in Economics for his role adapting and popularizing this theory to the analysis of discrete choices such as travel mode choice.

\subsubsection{Random Utility Maximization (RUM) Theory}

Random utility maximization (RUM) assumes that, when making a choice from a set of discrete alternatives, the decision-maker always selects the best alternative for himor her-self, the one with the highest utility (a scalar measure of value). If every attribute considered by the decision-maker were known to the analyst for every alternative, a RUM-based discrete choice model could be developed to predict with certainty every choice. However, the utilities of alternatives are not known to the analyst with certainty, so a portion of each utility is assumed to be a random variable. RUM theory provides a mathematical means to account for these random components of utility when predicting discrete choices. Note that RUM assumes a rational decision-maker and a reasoned decision-making process. This means that the decision-maker will always choose the best 
alternative given the information available to them at the time, and that given the same exact conditions the decision-maker will always make the same choice.

RUM's roots are actually in a mathematical branch of psychology and date back nearly 100 years. They merge with economic utility theories dating back even earlier (Dupuit, 1952; McFadden, 2007). The law of comparative judgment (Thurstone, 1927) introduced what would be called RUM through the use of imperfect discrimination between stimuli, called the discriminable dispersion model. One might recognize similarities with RUM in that true values along an artificial psychological scale are perceived with random and normally-distributed error. Using a formula for the probability that one alternative is better than another, Thurstone derived what is now called the binomial probit model (McFadden, 2001).

The term "random utility maximization" was coined by Marschak (McFadden, 2001) when Thurstone's theory was brought into an economic utility framework and generalized for more than two alternatives (Block \& Marschak, 1960; Marschak, 1960). Around the same time, Luce postulated a choice axiom of individual behavior that suggests the ratio of choice probabilities remains constant no matter the other choices, terming this "independence from irrelevant alternatives" (IIA) (Luce, 1959, 1977). IIA is a well-known property of simple discrete choice models such as multinomial logit. Others later proved that choice probabilities consistent with RUM would need to assume error terms are independently and identically distributed Type I extreme value in order to satisfy the IIA axiom (Luce \& Suppes, 1965). McFadden then derived the conditional or multinomial logit model from Luce's axiom (McFadden, 1973). 
With RUM theory developed into an estimate-able model form, applications exploded, with much work being focused on developing disaggregate techniques to analyze individual travel behavior. Some of the first applications of RUM to travel demand modeling and planning were in the Pittsburgh (Domencich \& McFadden, 1975; McFadden, 1973) and San Francisco (McFadden, 1974) regions. Among the many travel decisions analyzed with so-called discrete choice models were trip frequency, destination choice, time-of-day choice, and mode choice. Many generalizations of basic discrete choice models that fall within random utility maximization theory have been proposed (Manski, 1977) and used to analyze travel behavior and to model travel demand.

\subsection{Geography Theories}

In the 1970s, perspectives from the field of geography also strongly influenced conceptualizations of travel behavior and especially methods of analyzing and forecasting the demand for travel (Van Acker, van Wee, \& Witlox, 2010). In particular, a shift of focus from measuring and predicting travel patterns to looking at activity patterns that generate the demand for travel proved transformative. The contributions of Hägerstrand's time-space prism to describe temporal and spatial constraints on individual behavior (Hägerstrand, 1970) and Chapin's framework to explain individual activity patterns (Chapin, 1974) were foundational to the development of the new generation of activitybased travel demand forecasting models. 


\subsubsection{Time-Space Prism}

Hägerstrand contributed to travel and activity behavior theory through his seminal introduction of the time-space prism (Hägerstrand, 1970). In addition to suggesting that the regional science and geography fields should explain aggregate patterns through studying individual behavior, Hägerstrand proposed that individuals' behavior could be described in both time and space, bounded by three kinds of constraints: capability, coupling, and authority constraints. A primary capability constraint is the time-space prism, the temporal-spatial area within which an individual can travel to conduct daily activities (see Figure 2-1). This prism is bounded by the necessity of rest/sleep and the technological capabilities of transportation modes; an individual cannot appear beyond the boundary. For example, for two people with otherwise equal temporal-spatial constraints, the person riding a bicycle has a larger time-space prism than the person walking. Coupling constraints define when, where, and how individuals must conduct activities jointly in time and space. The selection of activities or "bundles" like work, where multiple individuals' time-space paths converge, necessarily limits the remaining available time-space prism. For example, if a person decides to walk two miles to and from work instead of driving, they theoretically reduce their opportunities for doing other activities. Telecommunications allow individuals to conduct some activities without spending time to traverse space. Authority constraints include laws, organizations, locations, or other domains in which activity bundles are organized and/or access is limited. For example, a person driving a motor vehicle along a freeway is limited to staying on the roadway and traveling in the legal direction. 
The work of Hägerstrand (1970) has been described a major contribution to the development of activity-based travel demand models (Ortúzar \& Willumsen, 2011), particularly for his focus on individual behavior and for introducing the concepts of constraints using the time-space prism. Activity-based models have a much stronger focus on feasibility, where primary choices (primary tour purpose and mode) constrain the available alternatives for secondary choices (number and purpose of stops, trip mode choice). For the purposes of the theory described in this thesis, Hägerstrand's most relevant contributions are: 1) the emphasis on explaining aggregate behavior through understanding individual behavior; and 2) the definition of feasible travel and activity alternatives based on constraints: intra-household interactions, other scheduled activities, and the capability constraints of time and space.

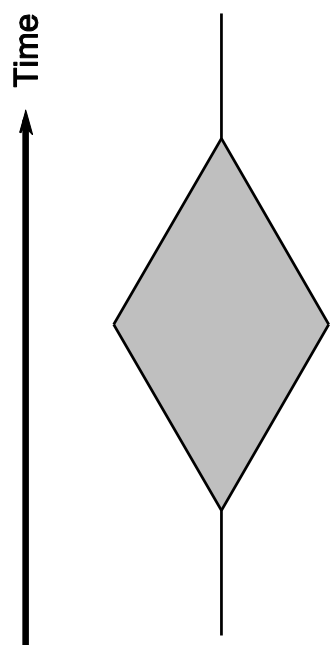

Walking

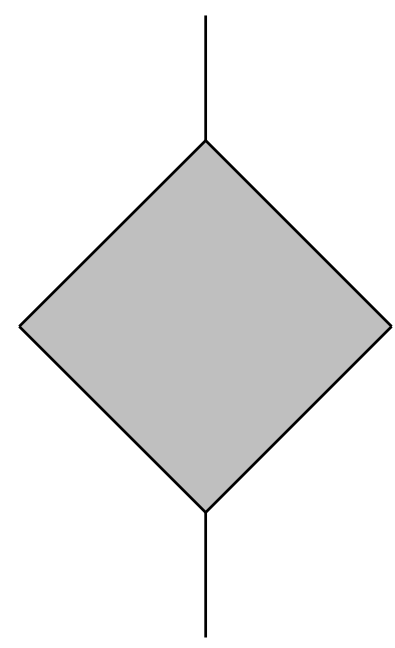

Bicycling

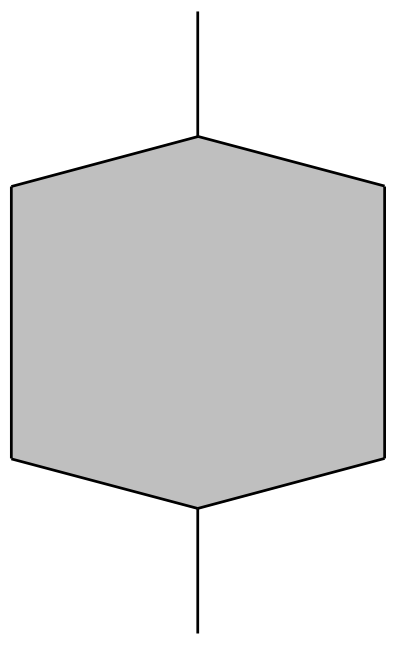

Driving + Activity

Distance

Figure 2-1: Time-Space Prisms

(Hägerstrand, 1970) 


\subsubsection{Activity Pattern Model}

Chapin's work contributed to travel behavior through his focus on and analysis of activities and activity patterns (Chapin, 1974). To explain individual behavior, Chapin suggested three components: motivation (fulfilling a want or need), choice (selecting between perceived and feasible alternatives), and outcome (action). Chapin outlined a general model for explaining aggregate activity patterns that captures individual behavior (see Figure 2-2). In the model, activity patterns are determined by two factors: propensity and opportunity to engage in an activity. Propensity is affected not only by constraining factors (like Hägerstrand), including roles and personal characteristics, but also by energizing or facilitating factors, including motivation and ways of thinking. For example, a person who is motivated to walk for exercise but constrained by her work schedule may have a lower propensity to walk. Chapin's model suggests that socioeconomic characteristics, in the form of individual constraints on propensity, influence activity patterns and thus travel. Opportunity is affected by both the availability and the quality of facilities and services. For example, a person may have a propensity to commute by bicycle, but without safe bicycle facilities along his path he has a low opportunity to bicycle. In this way, Chapin (1974) suggested that activity patterns (and underlying individual behaviors) are the result of both demand- (propensity) and supply(opportunity) side effects.

The activity pattern focus of Chapin's work has also had a seminal contribution to activity-based travel modeling, including his focus on both individual and aggregate levels. Chapin's analyses of activity patterns in the Washington, DC, area (Chapin, 1974) foreshadowed a key component of activity-based models: the choice of a daily activity 
pattern. For the purposes of the theory described in this thesis, Chapin's most relevant contributions are: 1) his findings that personal characteristics like demographic and socioeconomic factors influence the feasibility of activities and travel through constraints; and 2) that environmental factors about the availability and quality of transportation facilities and services also influence activities and travel.

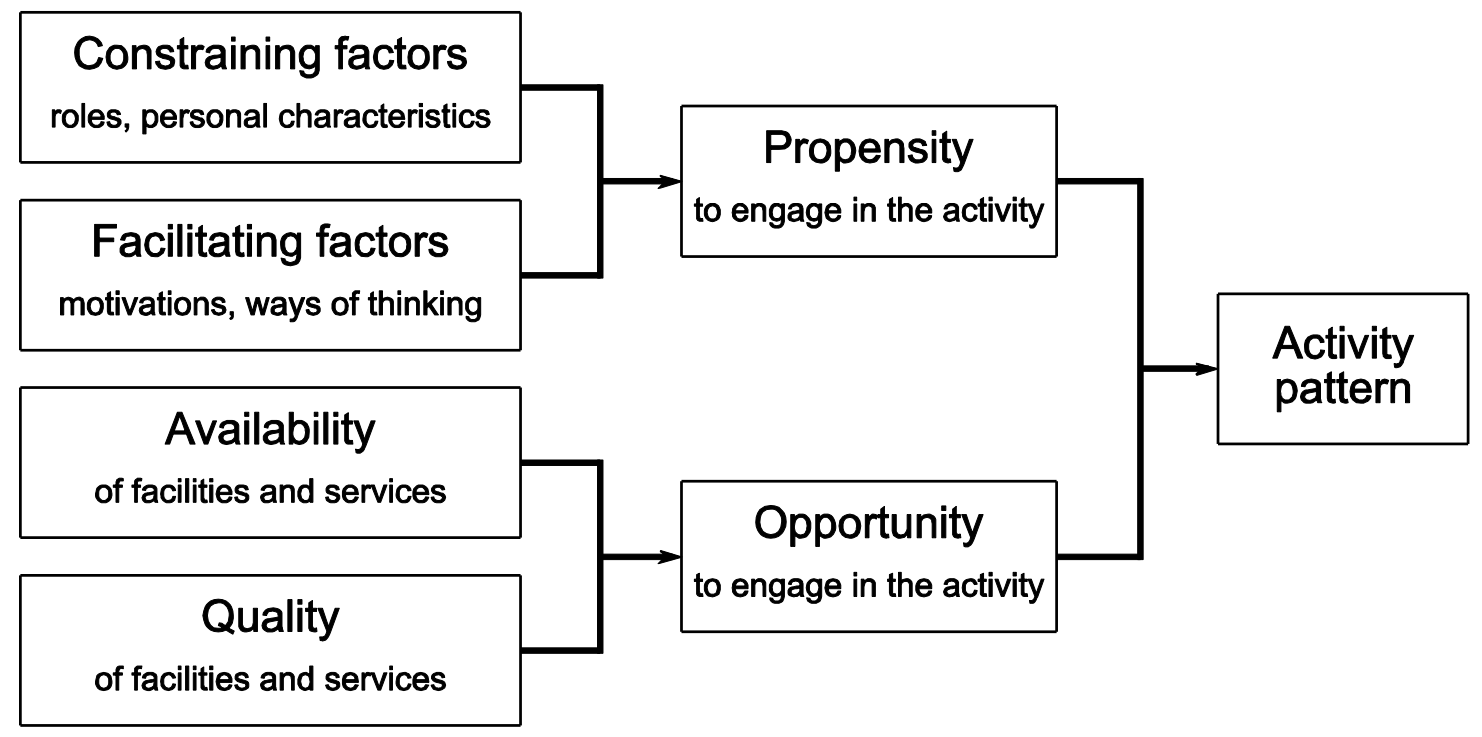

Figure 2-2: Activity Pattern Model

(Chapin, 1974)

\subsection{Psychological Behavior Theories}

Recently, there has been an increasing recognition that, in addition to standard economic factors like time and cost, non-objective individual factors like perceptions, attitudes, beliefs, and preferences may play key roles in travel decision-making processes. These new paths in travel behavior research have mirrored and followed developments and generated increasing interest in shifting from aggregate population-level to disaggregate household and individual levels of analysis for travel demand. Whether 
motivated by the desire to explain otherwise random taste variation within a RUM framework or a desire to affect healthy behavioral change through encouraging increased levels of walking and bicycling travel behavior, researchers have turned to psychological theories to influence or guide their work, particularly in the fields of cognitive, environmental, and social psychology.

The following sections describe some of the basic psychological theories that may be applicable for the study of travel behavior, particularly as related to factors and processes influencing walking and bicycling. The theory of human motivation notes how human behavior is motivated by certain basic needs. Frameworks like the theory of reasoned action, the theory of planned behavior, the theory of interpersonal behavior, the theory of repeated behavior, and the integrated behavioral model describe the many factors that influence behavioral intention. Social cognitive theory postulates relationships among behavior and many other factors. Finally, theories like the transtheoretical model, the ipsative theory of behavior, the normative decision-making model, and the comprehensive action determination model lay out staged processes of decision-making by which many of these factors lead to behavioral choices or behavioral change.

\subsubsection{Theory of Human Motivation (THM) or Hierarchy of Needs}

The theory of human motivation (THM) (Maslow, 1943; Maslow, 1954) introduced the pyramid or hierarchy of needs, one of the most influential concepts in psychology (Kenrick, Griskevicius, Neuberg, \& Schaller, 2010). THM presents several hierarchical needs that motivate humans to act towards fulfillment of a need. Maslow was 
careful to note that motivation is only one major factor, along with biological, cultural, and situational influences, in determining behavior. In THM, five basic needs are arranged into a hierarchy of prepotency (see Figure 2-3) such that lower ones usually must be fully satisfied prior to higher level ones dominating motivation. The lowest, most basic, human needs are physiological: homeostatic needs to maintain the body, including food. Next, higher-order needs emerge: safety, love, esteem, and self-actualization. The safety needs include preventing illness and avoiding dangerous and life-threatening situations. The love needs include affection and belongingness to other humans. The need for esteem reflects desires for self-confidence and appreciation. Finally, the need for selfactualization can be described as happiness or meetings one's potential. Maslow also noted that gratified needs are no longer motivational.

More recently, some scholars have reconsidered and expanded Maslow's hierarchy of needs from a functional or evolutionary perspective, linking goals or needs to outcomes. They suggest that basic human motivations include physiological, selfprotection and safety, affiliation and belongingness, status and esteem, and mate and parental needs, but not self-actualization (Kenrick et al., 2010). In the transportation realm, Maslow's hierarchy of needs has found minor applications in analyzing the motivations of tourist's travel behavior and destination marketing (Pizam \& Mansfield, 1999) and in at least one study on telecommuting (Salomon \& Salomon, 1984). One author has even adapted this theory to explain walking behavior through a hierarchy of walking needs (Alfonzo, 2005); this work is discussed in a later section.

For the purposes of the theory of travel decision-making, the primary takeaways from the theory of human motivation (Maslow, 1943, 1954) are surrounding the 
hierarchy of needs. Specifically, THM suggests that (travel) behavior is motivated by (travel and other) needs. Furthermore, the hierarchy of prepotency proposes that different considerations motivate behavioral decisions, and that they may act sequentially. The hierarchy of travel needs, part of the theory described in this thesis, will diverge from Maslow's THM in that activities (and a desire to engage in them) and not needs motivate travel behavior. Of course, Maslow's basic human needs may indeed motivate people's demand for activities and thus for travel. The hierarchy of travel needs will also consider that travel needs may instead act collectively, albeit with greater consideration weight placed on some than on others. 


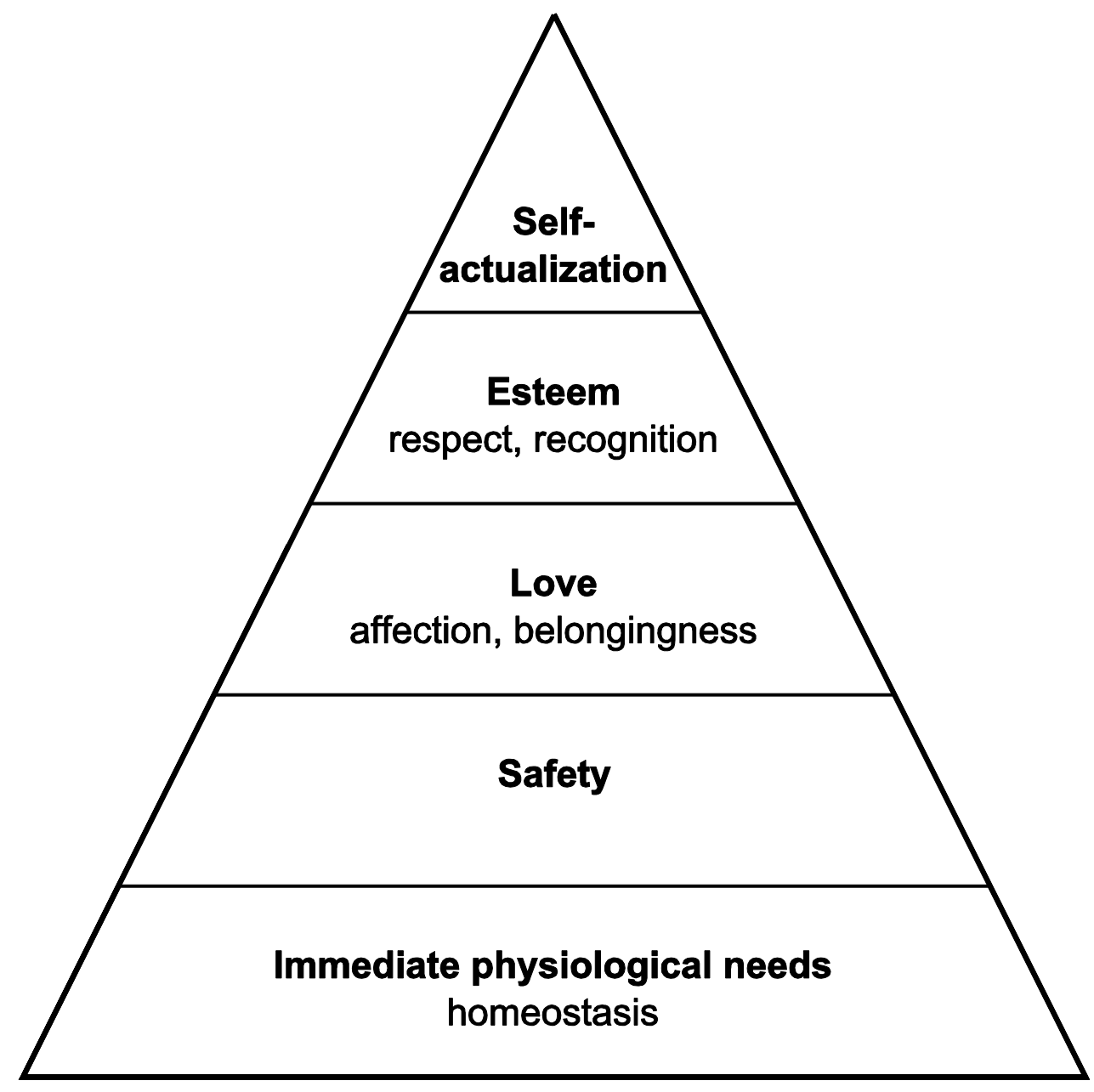

Figure 2-3: Hierarchy of Needs for Human Motivation

(Kenrick et al., 2010; Maslow, 1943, 1954)

\subsubsection{Theory of Reasoned Action (TRA)}

The theory of reasoned action (TRA) (Fishbein, 1980; Fishbein \& Ajzen, 1975; Montaño \& Kasprzyk, 2008) was developed to explain how attitudes could relate to behavior (see Figure 2-4). Like THM, TRA postulates that a motivation or an intention to perform a behavior is the biggest driver of actual behavior. For example, a person's 
observed behavior of walking to the store is determined by his intention to do so. This behavioral intention is directly influenced by attitudes towards the behavior and subjective norms (or social pressure) regarding the behavior. For example, a person's intention to commute by bicycle is shaped by her attitude towards bicycling and subjective norms about bicycling. In turn, attitudes are affected by the strength of normative beliefs about behavioral outcomes and the positivity of evaluations about behavior outcomes. For example, a person who strongly believes that walking will improve his health has a positive attitude toward walking. Subjective norms are affected by the strength of beliefs about whether others approve of the behavior and the strength of motivations to meet others' expectations of performing the behavior. For example, a person who believes her friends think she should commute by bicycle and is motivated to comply with their expectations has a positive subjective norm about bicycling. Demographic, socioeconomic, environmental, and other factors are hypothesized to operate only indirectly though their influence on other model constructs. For example, people with lower incomes may have stronger beliefs that others disapprove of bicycling than do people with higher incomes.

TRA has been applied in many different settings, including in travel behavior analysis. Its most valuable contribution is suggesting that the relationship of attitudes and social norms on travel behavior is mediated by intention. This suggests a role for attitudes, beliefs, and perceptions in the modeling of travel behavior choices. A growing body of work has investigated the role of attitudes on travel behavior (e.g., Gärling, Gillholm, \& Gärling, 1998). It is also important to note that, as with RUM theory, TRA assumes a rational actor or decision-maker and a reasoned decision-making process with 
volitional control. This is a significant assumption on the part of extant travel demand models and a limitation of TRA that motivated the development of alternative psychological theories of behavior.

\subsubsection{Theory of Planned Behavior (TPB)}

The theory of planned behavior (TPB) (Ajzen, 1991) was developed as an extension of TRA to account for behaviors with limited volitional control (see Figure 2-4). TPB postulates that factors outside of the control of the individual also affect intentions and thus behaviors; behavioral intention is directly influenced by behavioral attitudes, subjective norms regarding the behavior, and (now) perceived behavioral control, how much one thinks one can control the intended behavioral action. Perceived control is, in turn, affected by the strength of beliefs about the existence of outside factors that facilitate or inhibit the behavior and the perceived impact or power of those factors. For example, a person who believes stormy weather strongly and frequently inhibits his ability to commute by bicycle has a negative perceived control over bicycling. TPB suggests that perceived control, in addition to influencing behavioral intention, also moderates the relationship between intention and behavior; although little empirical evidence has supported this hypothesis (Montaño \& Kasprzyk, 2008).

TPB performs better than TRA in situations where volitional control is limited, such as travel behavior. In the theory of travel decision-making explained in Chapter 3, travel choices are influenced by many factors outside the control of the individual, including intra- and inter-household interactions, the built and natural environments, capability constraints (the time-space prism), and (while traveling) the decisions of other 
travelers. These factors influence the perception of behavioral control which in turn affects intention and travel choices. A series of studies successfully applied the theory of planned behavior directly to travel mode choice analyses and interventions (Bamberg, Ajzen, \& Schmidt, 2003; Bamberg \& Schmidt, 1998, 2003). The authors found significant influences of attitudes, subjective norms, and perceived behavioral control on intentions, and of intentions on behavior, supporting the TPB hypothesis that intention mediates these relationships with behavior.

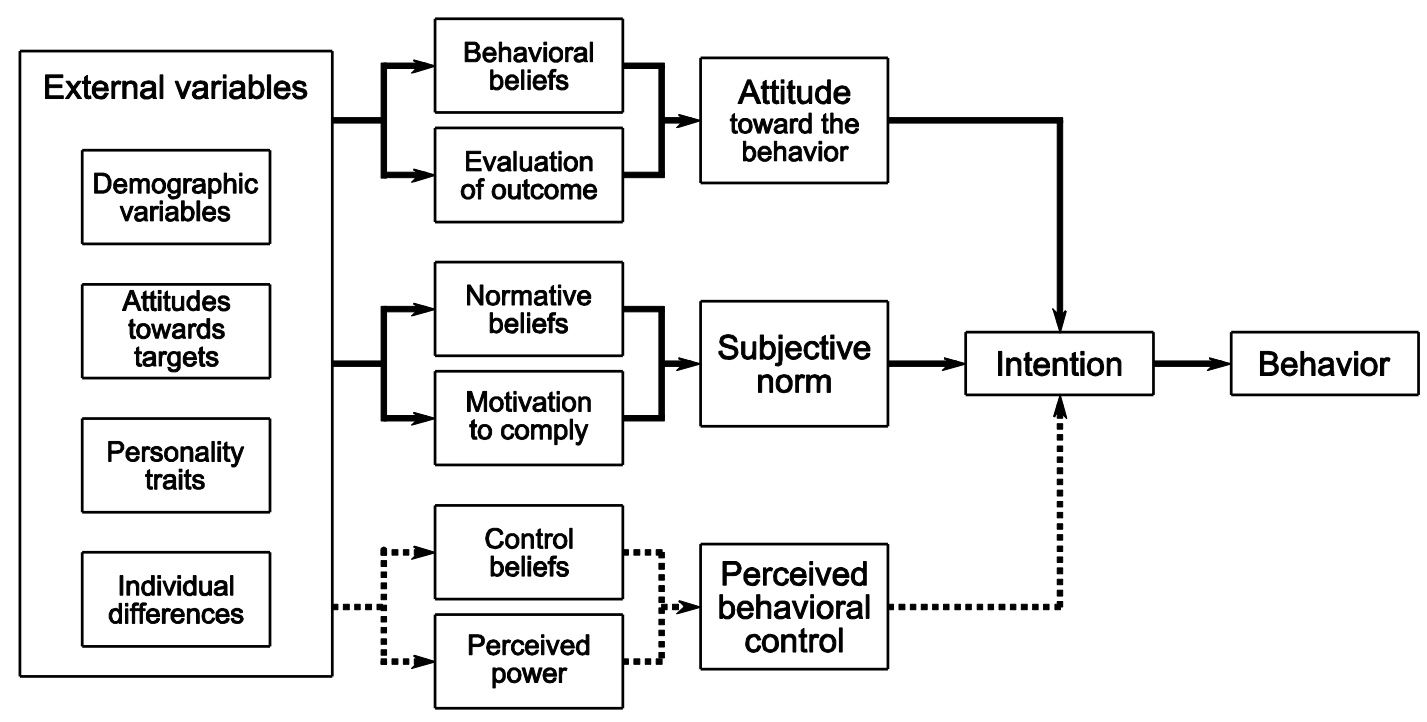

Figure 2-4: Theory of Reasoned Action and Theory of Planned Behavior

(Ajzen, 1991; Fishbein, 1980; Montaño \& Kasprzyk, 2008)

(additions for TPB shown in dashed lines)

\subsubsection{Theory of Interpersonal Behavior (TIB)}

The theory of interpersonal behavior (TIB) (Triandis, 1977, 1980) is very similar to TRA in that intention is the immediate antecedent of behavior (see Figure 2-5). In turn, intention is influenced by attitudes, social factors, and affective factors. Attitudes are 
defined in much the same as in TRA and TPB. Social factors include subjective norms of TRA and TPB, social roles (other's expectations about social position instead of behavior), and self-concept or beliefs about oneself. Affective factors are emotional responses to the behavior. In addition to these determinants of intention, TIB suggests that habits mediate the relationship of intentions on behavior. For example, a person may consciously intend to walk to the store but end up driving instead out of habit. Finally, context or facilitating conditions moderates the influence of intentions and habit. For example, a person may intend to walk to the store but end up driving instead because they must transport bulky goods.

Only a handful of transportation studies have interpreted and applied Triandis' theory to travel behavior research, but two have found empirical support for TIB. One study looked at mode choice using a multinomial logit model and found significant effects of attitudes, habits, and affective (emotional) appraisal on car and public transit use (Domarchi, Tudela, \& Gonzáles, 2008). A related study, using structural equations modeling and discrete choice modeling with latent variables, found the inclusion of attitudes significantly improved the model's explanation of mode choice (Galdames, Tudela, \& Carrasco, 2011).

One valuable contribution of Triandis (1980) is his formulation of an equation to predict the probability of an act:

$$
\begin{aligned}
\operatorname{Prob}(\text { Act })= & \left(\text { weight }_{\text {Habit }} \times \text { Habit }+ \text { weight }_{\text {Intention }} \times \text { Intention }\right) \\
& \times(\text { Physiological Arousal }) \times(\text { Facilitating Conditions })
\end{aligned}
$$

As applied to travel decisions, this suggests that the probability of choosing a travel mode alternative is a function of habit and intention, moderated by context (situational and 
environmental conditions). Habit plays an important role in the theory of travel decisionmaking, explained in the following chapter. Feasibility and environmental factors also figure significantly into the theory.

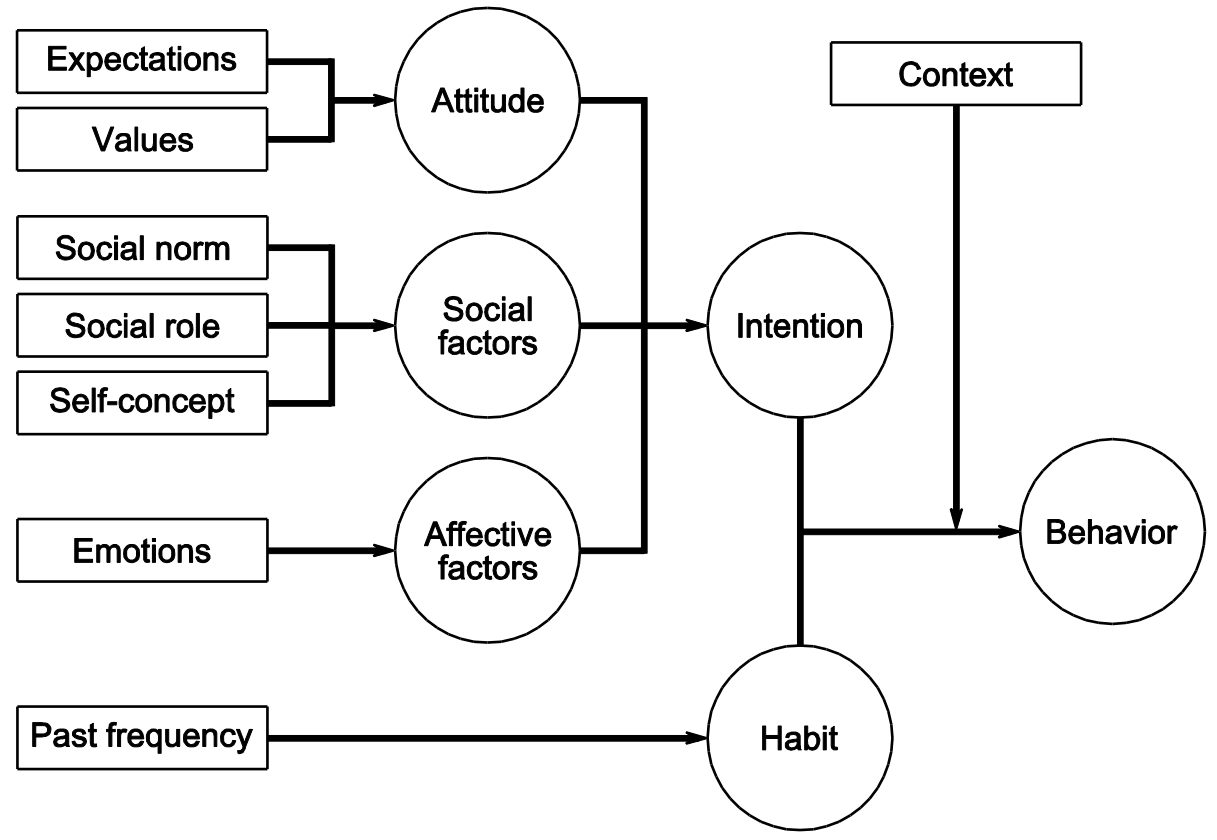

Figure 2-5: Theory of Interpersonal Behavior

(Domarchi et al., 2008; Galdames et al., 2011; Triandis, 1977, 1980)

\subsubsection{Theory of Repeated Behavior (TRB)}

The theory of repeated behavior (TRB) (Ronis, Yates, \& Kirscht, 1989) explains the roles of attitudes and habits on behavior that is frequently and extensively repeated (see Figure 2-6), and was developed to focus on health-related repeated behaviors. TRB proposes that behavior can be determined by either reasoned influences/decisions, as with behavioral intentions in TRA and TPB, or unreasoned influences, the primary of which is habit, as in TIB. While it is assumed that the original behavior was the result of conscious 
decision-making, and hence beliefs and attitudes, the theory suggests that habit is the greatest determinant of repeated behavior; while intention may drive the initiation of a behavior, its persistence is more affected by habit. For example, a person may drive to the local convenience store even though she could just as quickly walk or bike there simply because she has always driven there and does not consciously decide to change this habitual behavior. Stimuli or life changes can result in conscious decision-making and possible deviations from habitual behavior. For example, an informational campaign promoting walking may encourage a person to reconsider his habit of driving half a mile to work.

A primary contribution of the theory of repeated behavior is in explaining how habit informs repeated behavior; many travel decisions, particularly those related to commute behavior, fit the definition of repeated. Both intention and habit affect travel choices within the theory of this thesis. A number of other studies have found habit to significantly influence travel decision-making, such as mode choice (e.g., Aarts, Verplanken, \& van Knippenberg, 1997; Aarts \& Dijksterhuis, 2000). At least one study found support for the hypothesis that habits moderate the relationship between intention and observed mode choice (Gardner, 2009). Another study documented that interventions during a major life change such as residential relocation may affect a change in travel mode habits (Bamberg, 2006). 


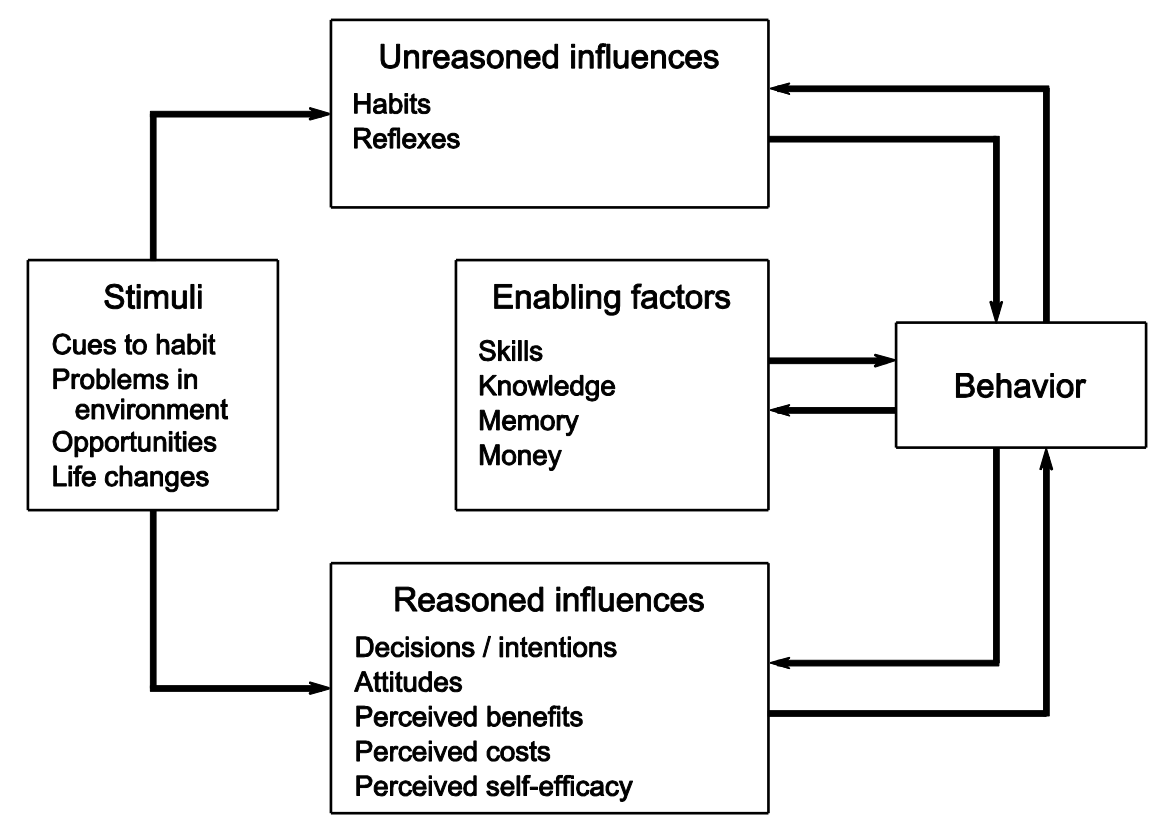

Figure 2-6: Theory of Repeated Behavior

(Ronis et al., 1989)

\subsubsection{Social Cognitive Theory (SCT)}

Social cognitive theory (SCT) (Bandura, 1986) is slightly different from other psychological theories in that it more broadly tries to explain nearly all human phenomena. SCT has been widely applied in the design of interventions to affect behavioral change. Developed out of theories to explain how people learn new behaviors, a core tenant of SCT is reciprocal determinism: the interaction between people, their behavior, and the environment (broadly defined, everything outside the individual). In SCT, behavior both influences and is influenced by personal, behavioral, and environmental factors (see Figure 2-7). Among psychological determinants of behavior are outcome expectations and self-efficacy. Outcome expectations and social outcome 
expectations correspond to attitudes and subjective norms from TRA and TPB. Selfevaluative outcome expectations are affected by how people think they will feel about themselves after performing a behavior. For example, a person may expect to feel proud of himself if he is able to leave the car at home and walk to the grocery store. The most widely-known concept of SCT, self-efficacy (Bandura, 1977) describes people's beliefs in their own ability to perform a behavior; self-efficacy is more important than outcome expectations for difficult behaviors. For example, a person who is confident she can ride her bicycle on streets without bicycle facilities has a high self-efficacy about bicycling. Self-efficacy is the genesis for perceived behavioral control in TPB (Ajzen, 1991). Among environmental determinants of behavior are incentive motivation and facilitation. Incentive motivation is the provision (through external control) of "carrots" for desired behavior and "sticks" for undesired behavior. For example, a Safe Routes to School program may provide positive incentives for students to walk or bicycle through competitions and prizes. Facilitation is the provision (through empowerment) of situations and resources that enable a behavior to be performed. For example, an open streets event empowers people to try walking and bicycling in their own neighborhood by facilitating the opportunity. Other key concepts in SCT include psychological explanations for observational learning, self-regulation, and moral disengagement (McAlister, Perry, \& Parcel, 2008).

Social-cognitive theory has been widely used in the public health field to examine individual, social, and environmental effects on health promotion and physical activity via active travel (Booth, Owen, Bauman, Clavisi, \& Leslie, 2000; Cunningham \& Michael, 2004; King et al., 2000; Lee \& Moudon, 2004; Troped et al., 2001). Its primary 
contribution for transportation research is the assertion that personal and environmental factors influence behavior. SCT has yet to be applied to considerations of walking and bicycling within a broader framework of travel decision-making or outside of health literature, with limited exceptions (Collantes \& Mokhtarian, 2007). As with other ecological theories, the influence of SCT appears in the theory of travel decision-making with the inclusion of environmental factors influencing travel choices.

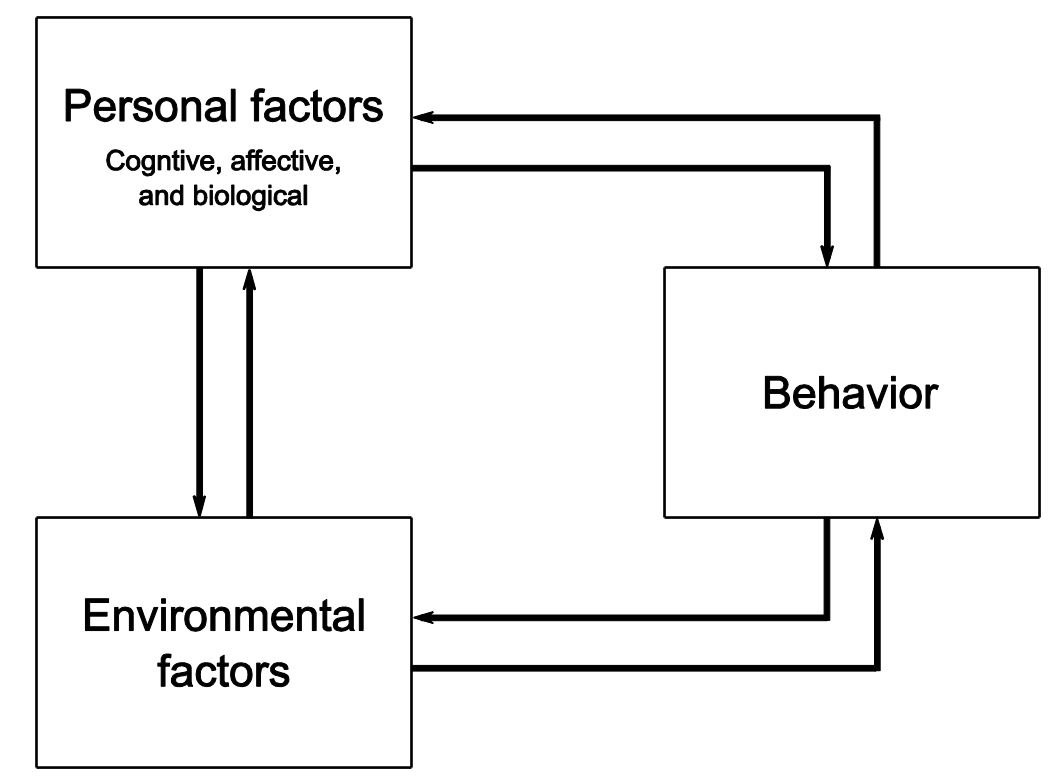

Figure 2-7: Reciprocal Determinism in Social Cognitive Theory

(Bandura, 1986)

\subsubsection{Integrated Behavioral Model (IMB)}

The integrated behavioral model (IMB) (Montaño \& Kasprzyk, 2008) is a further extension of TRA and TPB that also incorporates constructs from other behavioral theories, including TIB and SCT (see Figure 2-8). Although intention is still the driving 
force behind behavior in IMB, there are four other factors: knowledge of and skills to perform the behavior, salience of the behavior, environmental constraints that may prevent the behavior, and previous experience or habit performing the behavior. Both environment and habit are core concepts from TIB. Although attitudes, norms, and personal control still influence behavioral intentions, IMB adds additional factors. To (instrumental) attitudes IMB adds emotional response to the behavior, or experiential attitudes, from TIB. For example, a person with a strong negative emotional response to walking will have a negative experiential attitude. To subjective (or injunctive) norms IMB adds perceptions of what others are doing, or descriptive norms. For example, even without direct peer pressure, a person with friends who regularly commute by bicycle has a positive descriptive norm about bicycling. To perceived control IMB adds confidence in personal ability to perform the behavior, or self-efficacy, from SCT. For example, a person with great confidence about riding her bicycle has a stronger self-efficacy about bicycling. The author knows of no transportation study that has explicitly used as its basis the integrated behavioral model as expressed by Montaño and Kasprzyk (2008). That said, because IMB is derived from other psychological theories, many aspects of IMB have been included in travel behavior analyses. 


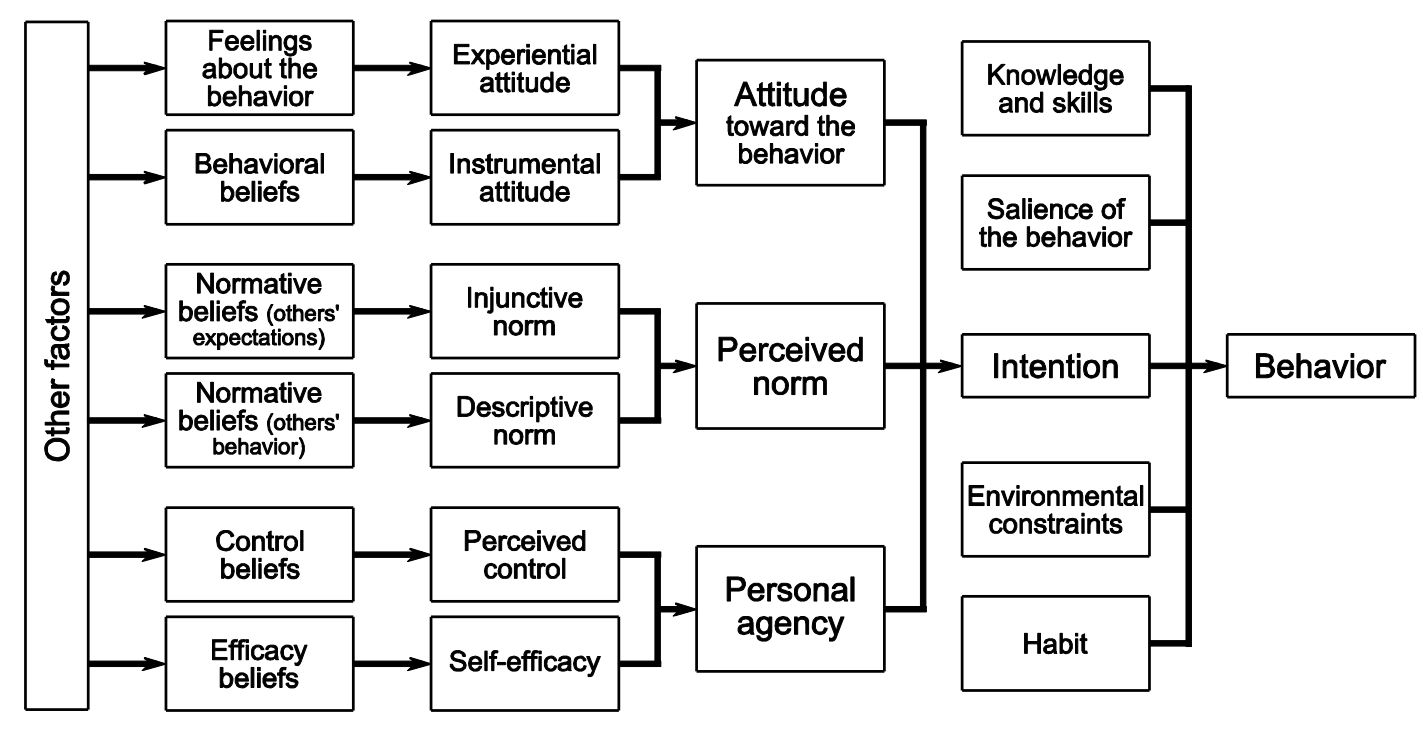

Figure 2-8: Integrated Behavioral Model

(Montaño \& Kasprzyk, 2008)

\subsubsection{Transtheoretical Model (TTM)}

The transtheoretical model (TTM) (Prochaska \& Velicer, 1997; Prochaska, 2008; Prochaska, Redding, \& Evers, 2008), like SCT, has found its broadest application in the design of interventions to affect healthy behavioral change, particularly away from highrisk behaviors. It attempts to explain behavioral change by describing six stages and ten processes of change. The stages of change reflect different time windows within which people intend to take action to change their behavior (precontemplation, contemplation, and preparation), have taken action or changed their behavior (action and maintenance), and shown no chance of relapse (termination). For example, the following people are in different stages of behavioral change: a person who thinks he should eventually try commuting by bicycle (contemplation); a person who has purchased a bicycle and associated gear (preparation); and a person who has been commuting by bicycle regularly 
for nearly a year (maintenance). Processes of change demonstrate how people move between stages; some processes are more commonly associated with specific stages of change. Processes include: consciousness raising (awareness), dramatic relief (emotions), self-reevaluation (self-image), environmental reevaluation (role model), self-liberation (willpower), social liberation (opportunities), counterconditioning (substitutes), stimulus control (prompts), contingency management (reinforcement), and helping relationships (social support).

The transtheoretical model has been widely used to design interventions that seek to improve health through increased physical activity, including walking and bicycling for transportation (Marshall \& Biddle, 2001; Sallis et al., 2006), with conflicting reports of success (Adams \& White, 2003; Hutchison, Breckon, \& Johnston, 2009). Others have investigated TTM's use in affecting mode shifts away from driving (Bamberg, 2007; Cooper, 2007).

In the theory of travel decision-making presented in Chapter 3, relevant constructs from TTM are assumed to act outside the boundary of concern. In other words, because the focus of TTM is on explaining multi-stage behavioral change, and not behavioral choice, it does not fit well within a short-term travel decision-making framework. However, understanding the process implied by TTM is useful when considering some of the inputs to the theory of travel decision-making, including defining which travel choices are feasible, especially based on individual perceptions, and in investigating the effects of habit or maintenance. For example, TTM may be a useful theory to first develop ways to change the shares of the "four types of cyclists" (Geller, 2006; Dill \& 
McNeil, 2013) among a population. These shares (or measures related to them) may then be used as inputs to components within the theory of travel decision-making.

\subsubsection{Ipsative Theory of Behavior (ITB)}

The ipsative theory of behavior (Frey, 1988; Tanner, 1999) (ITB) is a theory that was developed out of the behavioral economics field but has seen some adoption within an environmental psychology framework. Basically, ITB seeks to explain anomalous behavior that an economist might otherwise term irrational. From a microeconomic perspective, it distinguishes between an objective possibility set and an individual's subjective or ipsative possibility set of alternatives from which to choose; this is similar to the distinction between universal choice set and consideration choice set. In environmental psychology, this terminology is translated into three kinds of constraints on behavior or for the selection among alternative behaviors. Objective constraints, which comprise all of Hägerstrand's capability, coupling, and authority constraints (Hägerstrand, 1970), define the objective possibility set. Ipsative constraints, those factors that limit the mental/rational consideration of certain alternatives, define the ipsative possibility set. Once an ipsative possibility set is defined, subjective constraints, or preferences, are responsible for eliminating considered options after the evaluation of alternatives.

ITB has found few adoptees, and perhaps only one study has applied ITB in a transportation framework (Tanner, 1999). However, there are many aspects that may be of use to a comprehensive theory of travel decision-making. First, the idea of constraints defining an objective choice set may be useful in determining the feasibility of travel 
options. Next, ipsative constraints could be interpreted as those factors leading individuals to perceive the feasibility of those remaining travel options. Finally, subjective constraints will be reinterpreted as environmental factors that play into the evaluation of travel alternatives, seen through the filter of individual perceptions.

\subsubsection{Normative Decision-Making Model (NDM)}

The normative decision-making model (NDM) (Schwartz \& Howard, 1981) presents a theory to explain how altruism and non-altruistic factors affect normative decisions and helping behavior; environmental protection can be seen as an altruistic behavior. It is based on earlier work defining a conflict theory of decision-making (Janis \& Mann, 1977). Similar to TTM, NDM proposes four primary sequential stages leading from situational stimulus towards behavior (or inaction): attention, motivation, evaluation, and behavior. To proceed through the attention stage, one must be aware of a need (or be aware of consequences of inaction), identify actions to relieve the need, and recognize one's ability to execute the actions. For example, a person may be aware of the environmental benefits of bicycling to work and know how to go about doing so, but fail to act because he thinks himself not physically fit to ride. In the motivation stage, one considers whether internalized values and personal norms, external values and social norms, or non-moral physical and material outcomes motivate one to act. For example, a person may be motivated to commute by bicycle if they have internalized a social value for environmental protection. Next, in the evaluation stage, one weighs the moral and non-moral benefits and costs of alternative actions, including inaction. Situational and personal factors are thought to influence this evaluation. For example, a person may 
weigh the moral benefits of not driving to a store versus the time costs of walking there instead. Finally, if evaluation yields a conclusive result, action or behavior is undertaken in the fourth stage. Otherwise, a fifth stage, defense, occurs in which prior stages are reinterpreted. For example, when in an evaluative conflict, a person may think his one action of walking instead of driving makes no difference and thus denies to identify effective actions. Later work (Klöckner, Matthies, \& Hunecke, 2003) added habit defined as a script mediating situational cues and behavior - to NDM (see Figure 2-9).

NDM has been used in the travel behavior field to examine behaviors that have motivational elements of altruism or environmental protectionism, including walking and bicycling. Studies have found that personal or social norms have significant influences on non-auto mode choice, and that habit can moderate the relationship between norms and behavior (Klöckner \& Matthies, 2004; Klöckner et al., 2003; Wall, Devine-Wright, \& Mill, 2007). Concepts in NDM are useful in defining the theory of travel decisionmaking: non-moral motivations include desire for activity participation, attention reflects an individual's perceptions of feasibility, and habit plays a key role in sometimes interrupting this process. In addition, similar to the evaluation stage, the theory suggests a decision-maker evaluates the objective and subjective costs and benefits of various travel alternatives. 


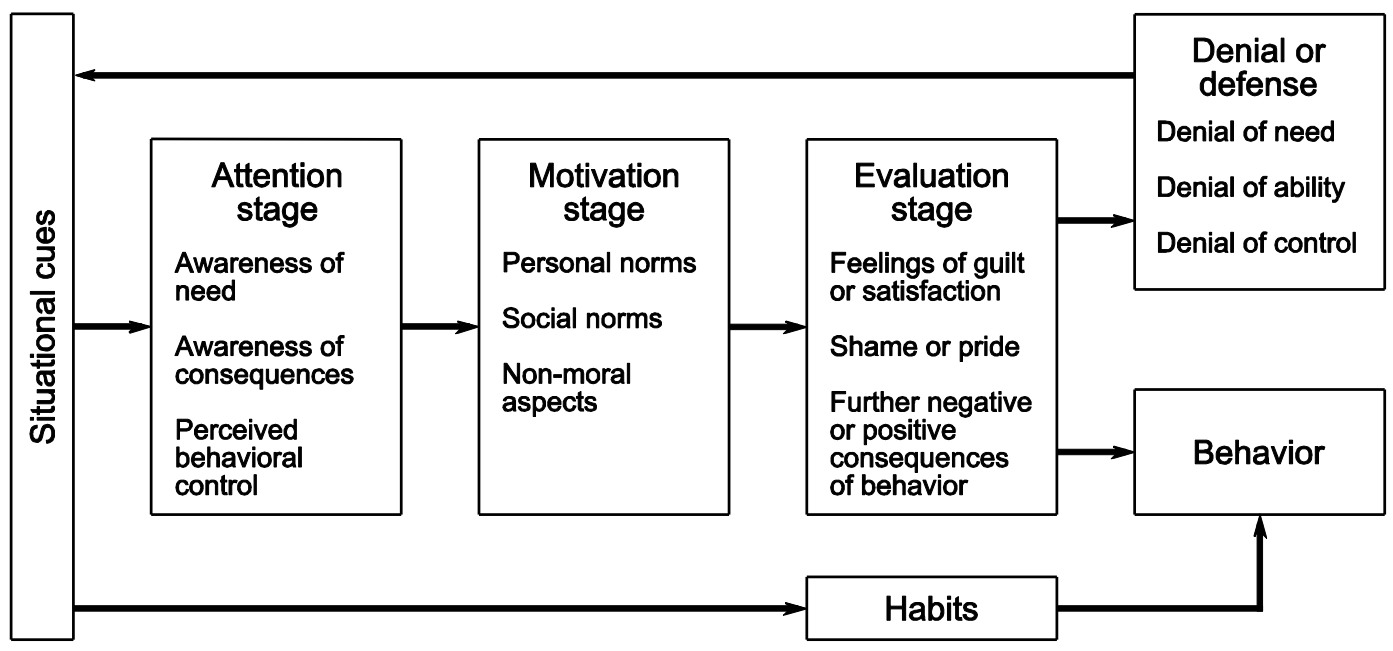

Figure 2-9: Normative Decision-Making Model

(Klöckner \& Matthies, 2004; Klöckner et al., 2003; Schwartz \& Howard, 1981)

\subsubsection{Comprehensive Action Determination Model (CADM)}

The comprehensive action determination model (CADM) (Klöckner \& Blöbaum, 2010) attempts to explain ecological behavior by integrating several behavioral theories and concepts, including TPB, NDM, TIP, and ITB (see Figure 2-10). CADM suggests three direct influences on behavior: habitual processes from TIP, intentional processes from TPB and NDM, and both objective and subjective situational factors from ITB. Habits and situations moderate the impact of intentions on behavior. Normative processes affect both habits and intentions. Situations affect normative, habitual, and intentional processes. CADM has been applied to the examination of mode choice among university students; normative, intentional, situational, and habitual factors were all shown to significantly affect mode choice (Klöckner \& Blöbaum, 2010; Klöckner \& 
Friedrichsmeier, 2011). These four concepts and their implied relationships in CADM will be useful for the development of a theory of travel decision-making.

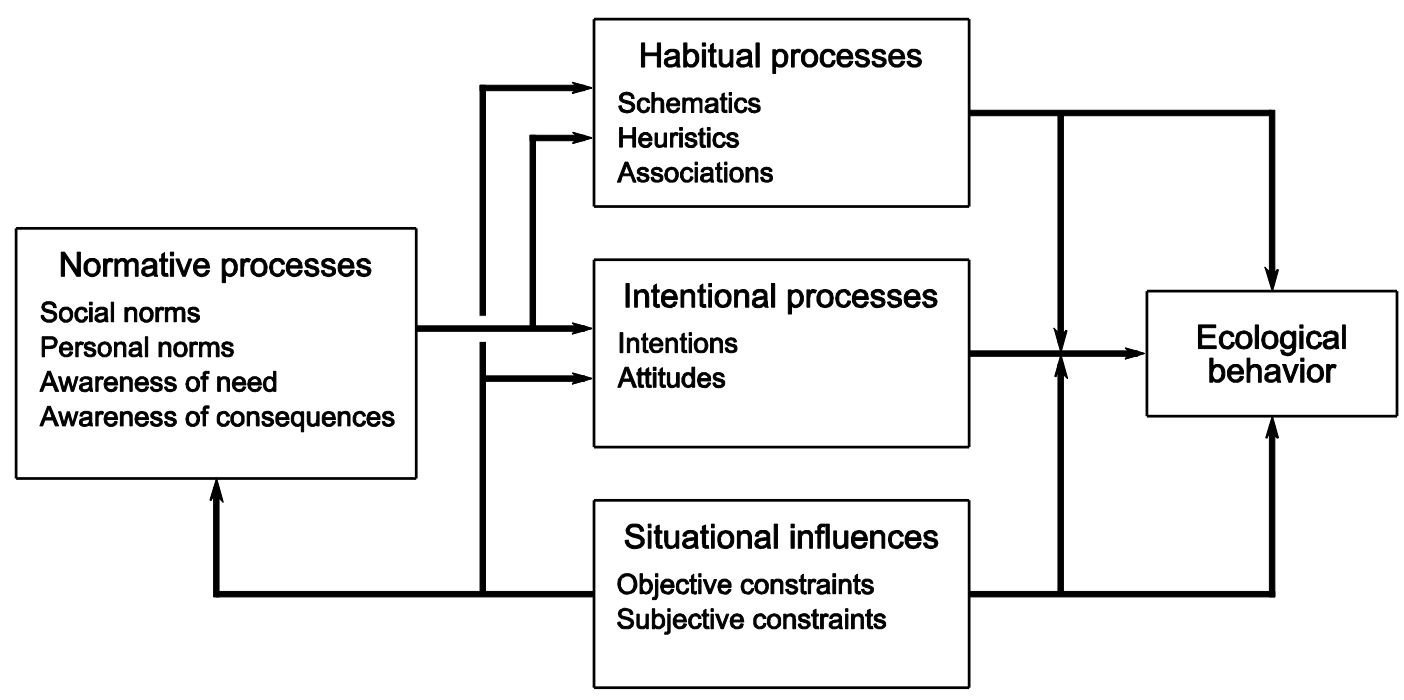

Figure 2-10: Comprehensive Action Determination Model

(Klöckner \& Blöbaum, 2010)

\subsection{Travel Behavior Theories}

In addition to general theories from the fields of economics, geography, and psychology, travel behavior-specific theories have been developed to explain the relationships and factors influencing transportation choices. Two theories, with a focus on walking and bicycling, are particularly useful for the development of a comprehensive theory of active travel decision-making. The hierarchy of walking needs (Alfonzo, 2005) proposes a social-ecological framework to explain factors and needs that determine walking behavior. The theory of routine mode choice decisions (Schneider, 2013) presents a process in which individuals consider different categories of similar factors when choosing between travel modes, including walking or bicycling. Together, 
components from two recent travel behavior theories form the core of the theory of travel decision-making presented in this thesis.

A number of other theoretical frameworks from the transportation, travel behavior, and public health fields have been proposed. Saelens, Sallis, and Frank (2003) proposed an ecological theory in which individual, environmental, and socialpsychological factors - many of which are included in the theory of this thesis influence walking and cycling. A similar framework was developed by Pikora, GilesCorti, Bull, Jamrozik, and Donovan (2003) with the addition of habitual behavior. Van Acker et al. (2010) presented a conceptual model of travel behavior that addresses many of the same issues as this thesis' theory - including activity behavior; individual factors; perceptions, attitudes, and preferences; and habits - as well as dealing with medium-term locational decisions, long-term lifestyle decisions, and social and spatial environmental contexts.

These other theories of travel behavior are not described in full here for several reasons. Some ecological models (Pikora et al., 2003; Saelens, Sallis, \& Frank, 2003) propose relationships between individual, social, and environmental factors and walking and bicycling, yet they may not be complete enough to explain travel by other modes. Furthermore, they are designed more for researchers and practitioners investigating ways to affect behavioral change (Sallis, Owen, \& Fischer, 2008) rather than transportation policy-makers interested in forecasting future travel. Other frameworks (Van Acker et al., 2010) are comprehensive and present many hypothesized relationships that are helpful for research design but require analytical tools (e.g., multilevel structural equations models) that are less useful for applications to travel demand forecasting tools. 


\subsubsection{Hierarchy of Walking Needs}

Alfonzo (2005) presented a "transdisciplinary, multilevel, theoretical model" (p. 817) within a social-ecological framework that explains how a variety of factors may affect walking. At its heart is a hierarchy of walking needs, based on the theory of motivation (Maslow, 1954), that organizes the needs people consider when deciding when and how much to walk. Figure 2-11 shows Alfonzo's framework, including the hierarchy of walking needs.

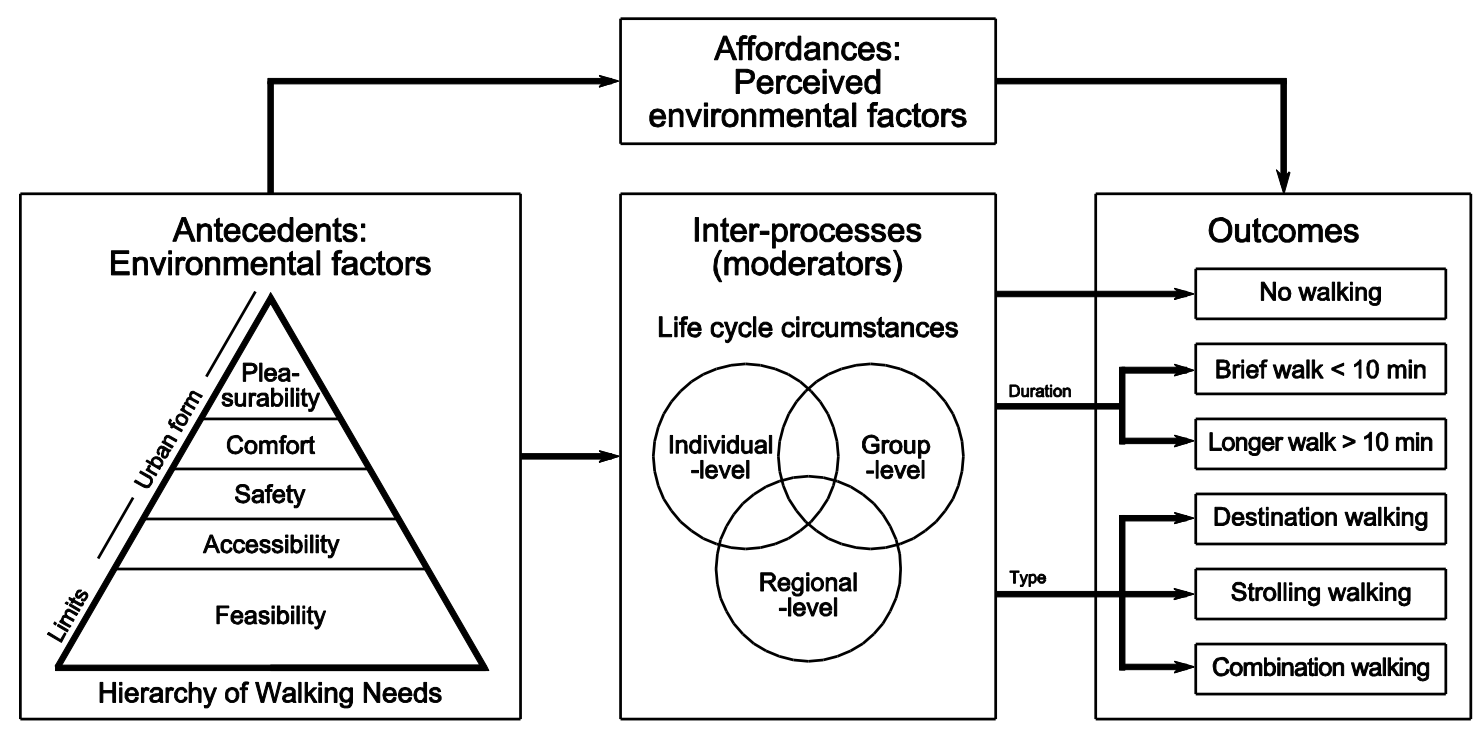

Figure 2-11: Social-Ecological Framework for Walking

(Alfonzo, 2005)

Feasibility is the lowest or most basic need within the hierarchy of walking needs: is walking viable for the type of trip under consideration? Factors affecting feasibility include mobility, time, and other responsibilities. Next is the need for accessibility, which pertains to the availability of and connectivity to activities. Accessibility factors include the number of destinations, their proximity, and any barriers in between. The third need is safety from the fear of crime, which can be affected by unmaintained physical features, 
certain land uses, and the presence of threatening people. Next is the need for comfort, broadly defined to include "ease, convenience, and contentment" (p. 828). Factors affecting comfort include barriers from motorized traffic, quality of the pedestrian network, and other urban design amenities. The final need is pleasurability, which pertains to the enjoyment or interestingness of the walking area. While the hierarchy is structured such that lower needs should be met prior to higher needs being considered, Alfonzo acknowledged that needs may be met out of order or considered simultaneously. Finally, the hierarchy only implies motivation, not that walking will take place; "the realization of these five needs is neither necessary nor sufficient to induce walking" ( $p$. 819).

The social-ecological framework of Alfonzo's model is focused on the hierarchy of walking needs but also includes other components and relationships. While the hierarchy is composed of many environmental factors that influence the fulfillment of needs, individual perceptions, habits, and motivations explain how different people view the affordance of these needs and make walking decisions. In other words, perceptions of the environment mediate the relationship between the hierarchy of needs and walking. Furthermore, Alfonzo identified several life-cycle circumstances that affect or moderate this relationship. Individual-level factors include demographic (age, gender, education), biological (weight), and psychological or cognitive factors (norms, attitudes, perceived behavioral control, etc.). Group-level characteristics include sociological and cultural factors. Regional-level factors include climate, topography, and geography. Put together, the framework suggests that perceptions mediate and life-cycle factors moderate the relationship between the hierarchy of needs (environmental factors) and walking. Finally, 
walking outcomes include choices among types of walking trips, walk duration, or not walking.

Alfonzo's theory contains many components that are particularly attractive for the comprehensive theory of general travel decision-making described in this thesis, inspired by considerations special to walking and bicycling. The characterization of specific antecedents, mediators, and moderators implies testable mathematical relationships between these factors and travel outcomes. Based on the above review of psychological theories, the hypotheses that needs motivate travel and that perceptions of the fulfillment of those needs moderate this relationship is consistent with other suggestions, including THM. It makes more theoretical sense for demographic and lifestyle characteristics to act as moderators rather than directly influencing travel. Finally, the hierarchy of needs appears to be in a reasonable order. Many of the components from this walking framework (Alfonzo, 2005) are incorporated into the theory of travel decision-making.

There are also limitations and challenges to the hierarchy of walking needs and Alfonzo's general social-ecological framework. The distinction among walking outcomes by three trip types and two duration categories is too simplistic. Safety from traffic might be better removed from the comfort need and added to the safety from crime need. Perhaps most significantly, there is no consideration of economic (or socioeconomic) factors - income, auto ownership, travel time or cost - that have been shown to influence the choice of travel modes (Ben-Akiva \& Lerman, 1985). Limited consideration is also paid to alternatives or substitutes for travel, including changing modes, time-of-day, destinations, frequencies, etc. The framework does not make clear how an individual goes about making a walking decision; the choice is divorced from motivating factors 
like the demand for getting to activities or exercise. Broadly, there is little consideration of the scale at which the hierarchy of needs acts and how it connects with related short(activity-scheduling) and medium-to-long-term (residential location, auto ownership) decisions. These limitations are to some degree addressed in the theory described in Chapter 3.

\subsubsection{Theory of Routine Mode Choice Decisions}

The theory of routine mode choice decisions (Schneider, 2013) describes how people may choose a travel mode for routine, non-work or school activities, such as shopping and other errands. Founded upon travel behavior research on the factors that influence walking and bicycling, it was developed with the support of qualitative in-depth interviews of shoppers traveling in the San Francisco Bay Area of California (Schneider, 2011). Schneider's theory is also based on a number of psychological theories, including TIB, TPB, TTM, NDM, and CADM. 


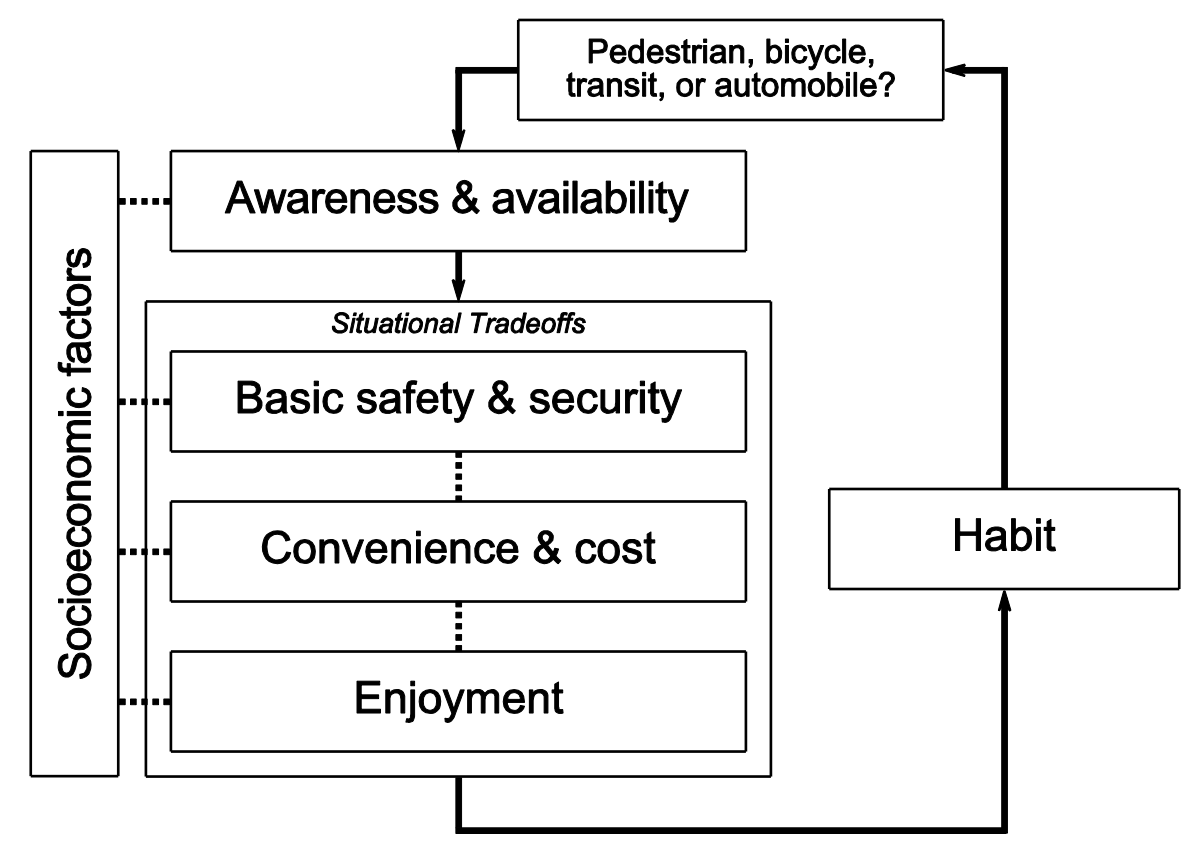

Figure 2-12: Theory of Routine Mode Choice Decisions

(Schneider, 2013)

Schneider's theory of routine mode choice decisions proposes a five-step sequential decision process, as shown in Figure 2-12. The first step, awareness and availability, determines the travel modes that are possible to be chosen and for which rational tradeoffs are subsequently made. In addition to factors like vehicle availability, people must be aware of a modal option in order to consider it. The second, third, and fourth steps of the theory are where situational tradeoffs between the considered modes occur, either sequentially or simultaneously. In the second step, travelers consider the safety that a given mode will provide from traffic collisions and the security that it may afford from crime. In the third step, people also tradeoff the convenience and cost of each mode, including money, time, and effort. The accessibility of activity locations and the price of parking were the two of the strongest factors determining walking and bicycling 
(Schneider, 2011). In the fourth step - enjoyment - individual, social, and global benefits may make certain travel modes more attractive. For example, walking or bicycling may provide health benefits from exercise. The fifth step, habit, lets prior choices influence future decisions through feedback and closure of the decision loop. Socioeconomic factors play an indirect role by influencing how individuals evaluate each of the first four steps.

Schneider's theory of routine mode choice decisions contains similarities with Alfonzo's hierarchy of walking needs. There is a reasonable hierarchical arrangement of similar modal considerations: first availability, then safety, cost, and enjoyment. Schneider's theory also makes some improvements on Alfonzo's hierarchy. Notably, the feedback mechanism accommodates unreasoned behavior and the influence of past mode decisions. In the theory, it is important that socioeconomic factors explain differences in response - moderation - rather than directly affecting mode choices.

A few components are not explicitly included in the theory of routine mode choice decisions. Perceptions, attitudes, and norms may also influence responses at each step in addition to socioeconomic factors. Other interrelated travel choices - residential location, auto ownership - and non-mode substitutes like time, destination, and frequency may also apply a variation of this decision-making process. Finally, there is only an implicit assessment of how these steps operate in a mathematical form that can be investigated through statistical hypothesis testing. These aspects are to some degree included and addressed in the theory of travel decision-making. 


\subsection{Conclusion}

The preceding sections have described some of the theories from the fields of economics, geography, and psychology that are influential in the study of travel behavior and the forecasting of travel demand, and that are particularly relevant to explaining active travel decision-making. Many theories show consistent and overlapping components and relationships. In part answering McFadden's call for better integration of economic and psychological theories of travel behavior into transportation planning models (McFadden, 2001), many of these concepts will be combined into a unifying theory of travel decision-making in Chapter 3.

Borrowing from the theory of human motivation (Maslow, 1943), travel will be described as derived from the demand for participation in activities (Chapin, 1974). Objective constraints (Frey, 1988), such as those expressed by the time-space prism (Hägerstrand, 1970), will define which multi-dimensional travel alternatives may be feasible. Subjective constraints, whether expressed as self-efficacy (Bandura, 1977), perceived behavioral control (Ajzen, 1991), or simply awareness (Schneider, 2013), will define which objectively-feasible travel alternatives are actually considered to be feasible by the decision-maker. Environmental factors, both facilitating and constraining (Bandura, 1986; Triandis, 1980), will also influence the evaluation of and choice from between travel options. Travel alternatives will be evaluated on the basis of a hierarchy of needs (Alfonzo, 2005; Maslow, 1954). Random utility maximization theory (McFadden, 1973, 2001) may still be the way these travel choices are analyzed, but other decision rules and processes (Prochaska \& Velicer, 1997; Schwartz \& Howard, 1981) could act instead. Important social and cognitive factors including attitudes and norms (Ajzen, 
1991; Fishbein \& Ajzen, 1975; Montaño \& Kasprzyk, 2008) will enter through their influence on the perception of values of alternatives or fulfillment of needs. Finally, measures of habitual behavior (Klöckner et al., 2003; Ronis et al., 1989; Triandis, 1980) will be able to influence and/or bypass the travel decision-making process. These constructs, derived from theory, will be further described and operationalized in the next chapter of this thesis, the elaboration of a unifying theory of travel decision-making with applications for modeling active travel demand. 


\section{THEORY OF TRAVEL DECISION-MAKING: A UNIFYING FRAMEWORK}

With further thought, these theories might add up to [a] complete whole. It is possible for researchers to start with one theory and borrow insights from the others... These theories all suggest that the relationship between the built environment and physical activity is not just a simple relationship between these variables and that the study of the relationship should be guided by a more comprehensive conceptual model. (Handy, 2005, p. 1-2)

\subsection{Introduction}

This chapter develops and describes the conceptual framework of a theory of travel decision-making, with specific emphasis on active travel modes: walking and bicycling. The components and relationships proposed specify one representation of the way in which people make travel decisions. This theory partially answers a call for better conceptual models to guide future studies on the effects on the built environment on active travel (Handy, 2005; Owen, Humpel, Leslie, Bauman, \& Sallis, 2004; Saelens \& Handy, 2008). It unifies travel behavior knowledge from a number of academic fields. As will be shown in Chapter 4, the theory also informs how transportation planning and policy-making tools - particularly regional travel demand forecasting models - might represent the complex influences on walking and bicycling. A conceptual framework of the theory is shown in Figure 3-1. 


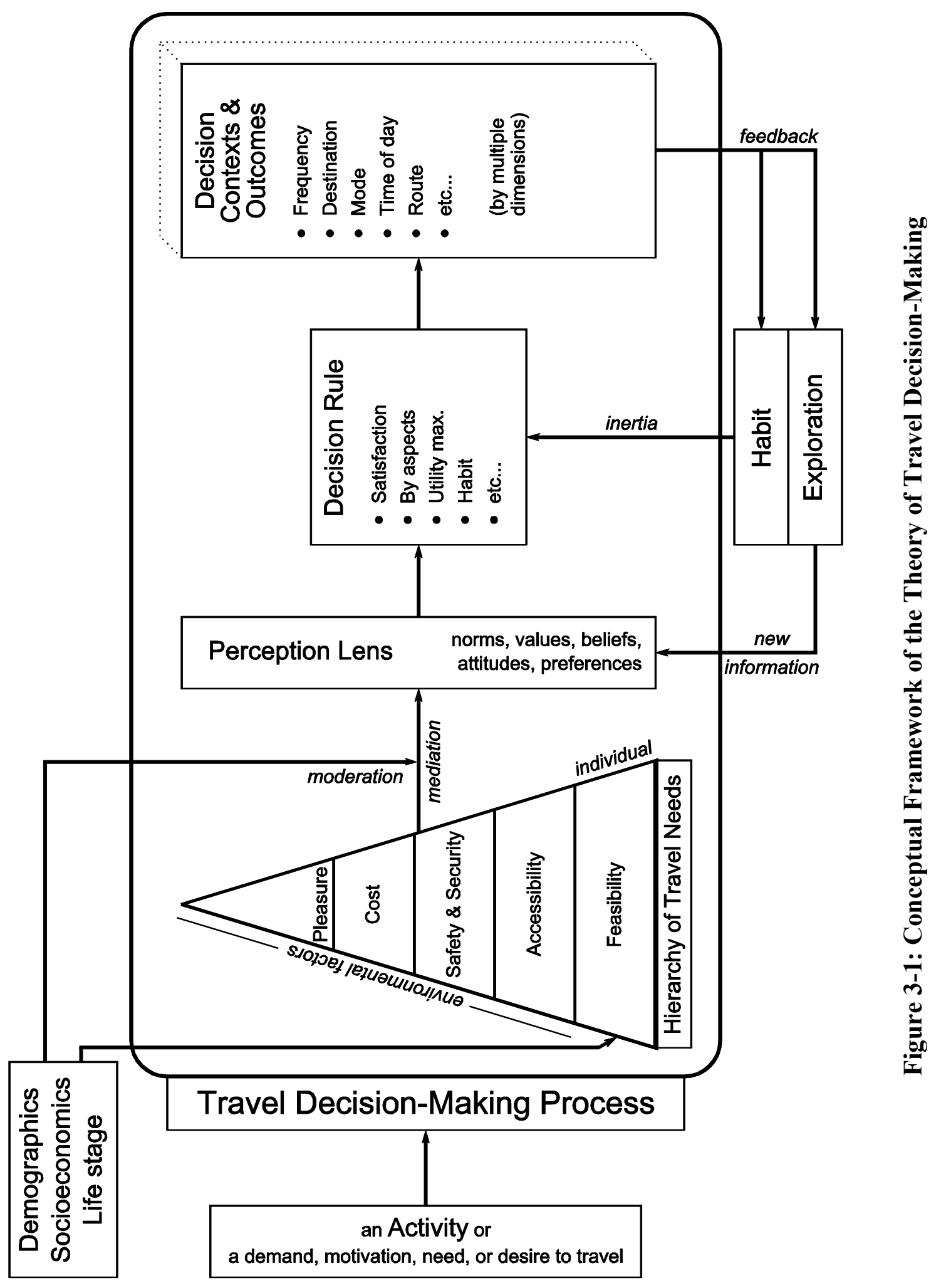


The theory of travel decision-making's major components and relationships are:

1. Activity: Demand for travel is derived from a demand for activities.

2. Hierarchy of Travel Needs: Categories of factors influence travel decisions.

3. Moderation: Demographic, socioeconomic, and life stage factors moderate.

4. Mediation: Individual perceptions of travel needs fulfillment mediate.

5. Decision Rule: People may use different rules to make decisions.

6. Decision Contexts and Outcomes: This theory applies to many, even multidimensional, travel decisions.

7. Feedback: Past travel outcomes influence the current decision-making process.

The theory was developed to capture and represent those factors specifically related to walking and cycling that have been hypothesized in some prior travel behavior theories (Alfonzo, 2005; Schneider 2013) but not yet adequately addressed in analyses and models of travel behavior, including the roles of safety and security, pleasure, and perceptions. However, it has broader appeal and is generalizable to all travel modes and most (if not all) travel decision contexts: mode, destination, route, etc. Depending on the context in which it is employed, some elements of the theory may change - such as the arrangement of the hierarchy of travel needs or the type of decision rule - but the overall conceptual framework should remain intact.

The theoretical framework of active travel decision-making has similarities with several other conceptual frameworks for walking, cycling, and physical activity, particularly ones from public health literature (Pikora et al., 2003; Saelens, Sallis, \& Frank, 2003). It also borrows considerably from the economic, geography, psychology, and travel behavior theories reviewed in Chapter 2, especially the hierarchy of walking 
needs (Alfonzo, 2005) and the theory of routine mode choice decisions (Schneider, 2013). In the following sections, inspirations of and deviations from these other theories will be noted.

This theory is hypothesized to act primarily in the short-run on day-to-day travel decisions by individuals such as choices of destination, mode, and route. It does not attempt to address activity decisions (although they may follow a related process), nor does it concern itself with other travel-related decisions such as choices of vehicle ownership, residential location, and lifestyle. These longer-term decisions are considered exogenous inputs to the theory, although it should be noted that people may evaluate a simplified version of the theory when making such decisions. The extent to which shortterm travel decisions influence medium-term location and long-term lifestyle decisions (Van Acker et al., 2010) is outside the scope of the theory.

It may be useful to define and distinguish between terminology common to travel behavior literature and which will be used to describe the theory of travel decisionmaking. An action is a thing done, whereas inaction is a thing not done. A decision is the result of coming to a conclusion about possibly acting. A choice is a decision when there are two or more options or alternative actions. A behavior describes the way in which a person acts. An outcome is a result or consequence of an action or inaction. The framework described in this chapter is a theory of travel decision-making because it accommodates decisions with only one option and allows for action as well as inaction.

The following sections describe each component (activity, the hierarchy of travel needs, demographics and socioeconomics, the perception lens, the decision rule, decision contexts and outcomes, and habit and exploration) and relationship (direct effects, 
moderation, mediation, and feedback) contained within the theory of travel decisionmaking. Within each section, the component and/or relationship is first defined. Next, where appropriate, theoretical support from the literature is described. Finally, where appropriate, examples of empirical evidence to support the component or relationship are noted in detail, summarizing the state of knowledge on the subject.

\subsection{Activity}

\subsubsection{Definition}

Activity participation is the underlying motivation for travel. People travel in order to do something. This is what is meant by travel being a derived demand: transportation is a means to an end, which is being in a different spatial location in order to conduct or participate in activities. From this perspective, travel can be viewed as a task to be minimized (in time, cost, or other expenditure). However, the lines between travel to activities, conducting an activity while traveling, and travel as an activity often blur (Mokhtarian \& Salomon, 2001), as discussed below.

The most common situation is where travel is an ancillary task with only the ultimate goal of moving the location of oneself for an activity. There is no benefit obtained from the act of traveling besides getting from one place to another. The personal travel action affords transportation to an activity destination but has no simultaneous activity value. In fact, travel usually imposes a cost, either in monetary terms or by reducing the time available for other activities. 
Often, travel can facilitate even in a small way other desires or activities which blur the boundary between getting places and doing things. For example, a person may find benefit from listening while driving to work. Driving meets this person's need for both getting to her final destination and listening to her favorite radio talk show or audio book; arguably the need to travel to the activity (work) dominates decision-making over the listening benefit, but both contribute. In another example, a worker may plan her day so as to conduct business while riding commuter rail during her hour-long journey home. Riding transit meets this person's need for both getting to her final destination and doing work; arguably the travel need dominates decision-making over the working need, but both contribute. Finally, consider a person who chooses to walk to the store as part of his weight-loss program. Walking meets his need for both shopping and exercise; arguably the need to shop dominates decision-making over the exercise benefit, but both contribute. In these cases, the travel action is accompanied by an activity, providing primary value as a means of transportation (including associated costs) but also some activity benefits.

Sometimes travel is itself the end; the activity is travel. A road trip may provide the multiple purposes of getting somewhere, seeing the country, socializing with family and/or friends, and relaxation. Cruising is an activity in itself where the benefits are socialization and status. Similarly, making an outdoor recreational trip may not get one to a different location (and usually begins and ends at the same place), but the act of walking, jogging, running, or cycling is also the activity of exercise. Substitutes for outdoor exercise do not involve travel. In the case of driver education, the entire purpose of the journey is to train oneself to learn how to safely operate an automobile. From these 
perspectives, the travel action is the activity; the activity value of the act of traveling outweighs others costs of transportation even without an associated benefit of getting somewhere.

This line between activity and personal travel is knowingly elastic. Consider the definition of activity as one in which a substitute that does not involve travel is unavailable. Given an activity, we construct our choice set as comprising solely travelbased alternatives. However, with enough flexibility, we can define most "choices" to meet activity demands as having non-travel options. Many people work from home or have the option to telecommute occasionally. Home-schooling has always been an option, and cybereducation is growing. Online shopping has exploded in popularity and even groceries can be purchased online and delivered. Even other activities which normally require an in-person interaction, such as routine health appointments, have nontravel telecommunications options like video meetings over the internet. The transportation field finds comfort concerning itself, especially when modeling, with seeing a perpetual demand for travel through people conducting activities. In reality, transportation is but one aspect of daily life, the demand for which varies with other technological and societal developments.

In the end, this theory suggests that travel is a derived demand based on a need, motivation, or desire to conduct activities, defined broadly. Activities here refer not only to typically-defined activities like work, school, and shopping, but also other general motivations like exercise and socialization. Anything that would be enough to induce someone to travel is considered an activity that initiates the travel decision-making process. Put another way, an activity is the primary motivator for travel, without which 
travel would not have taken place; activity demand is the driver of travel demand. In turn, the demand for activities is motivated by the fulfillment of basic human needs and wants (Maslow, 1943). This view is consistent with Chapin's (1974) framing of human activity patterns.

Note the one-way relationship between activity and the travel decision-making process. In reality, there may be interactions between activities and travel, particularly where a past travel decision constrains or facilitates future activities. For example, choosing to walk to the store may restrict one's ability to conduct other activities on the way home. However, these travel-activity interaction effects operate beyond the relatively narrow scope of the theory of travel decision-making. The theory assumes that an activity is selected first, thus driving a travel consideration. That said, a decisionmaking construction similar to this conceptual framework may determine the demand for activities.

\subsubsection{Theoretical Support}

The assertion that travel demand is derived from the demand for activities has received strong theoretical, empirical, and practical support. Hägerstrand (1970) discussed travel and activities as simultaneously constraining one another through timespace prisms. Similarly, Chapin (1974) and others began analyzing patterns of activity in urban areas and viewed travel behavior as bounded by participation in activities. Numerous other studies through the 1980s and 1990s expanded on these theoretical foundations using empirical research and implementing planning tools to develop what is known as the activity-based approach to represent travel behavior and forecast travel 
demand (Algers, Eliasson, \& Mattsson, 2005; Ben-Akiva \& Bowman, 1998b; McNally \& Rindt, 2008). Many models have been developed in a variety of different implementations (Axhausen \& Gärling, 1992; Jovicic, 2001; Ortúzar \& Willumsen, 2011), but they all use activity as the basis for deriving travel. Others note the ability for travel to be an activity in itself (Mokhtarian \& Salomon, 2001).

\subsection{The Hierarchy of Travel Needs}

\subsubsection{Overview and Operation}

Once an activity is demanded, the next step is to enter the travel decision-making process, the first stage of which is a consideration of the hierarchy of travel needs. The hierarchy of travel needs - portrayed in Figure 3-2 - is the core component of the theory of travel decision-making. It categorizes and ranks those attributes - personal, social, and environmental - that are hypothesized to drive the evaluative process of transportation decisions. The hierarchy defines characteristics of those needs, motivations, or intentions and attributes of alternatives that are of importance during travel decision-making. The five general categories of needs - feasibility, accessibility, safety and security, cost, and pleasure - are described in more detail in the following sections. 


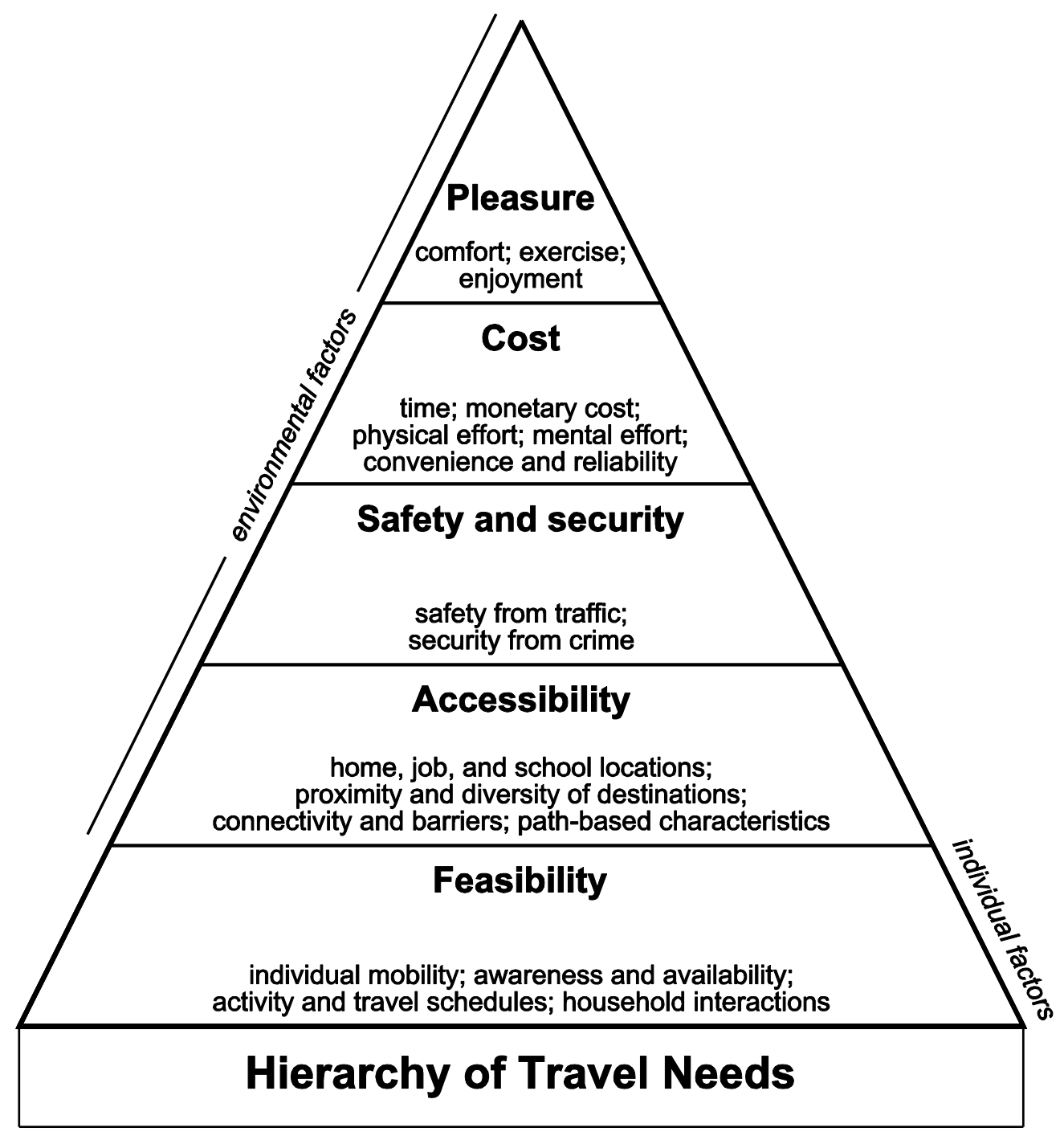

Figure 3-2: The Hierarchy of Travel Needs

In one way, this hierarchy can be conceived as a list of attributes or characteristics that define a particular (multi-dimensional) travel alterative. The "needs" are simply ways to categorize variables, including individual and environmental factors, which might be considered by transportation planners and policy-makers in a way that more closely represents a hypothetical behavioral process. When an individual makes a travel decision, the theory suggests that they evaluate these factors within the filter of their own 
perceptions, making evaluations of how well each of the needs is fulfilled. It is assumed that the decision-maker explicitly considers these factors, but this may not necessarily be true.

This formulation of the hierarchy of travel needs is congruent with many economic, psychology, and travel behavior theories. Most notably, it mirrors in scope and structure the hierarchy of needs that motivate human actions (Maslow, 1943, 1954). There are lower-order needs that dominate motivation or decision-making before or beyond higher-order needs. Similarly, the hierarchy follows from TRA (Fishbein \& Ajzen, 1975) in that motivation or intention is the primary driver of behavior. Fulfillment of these needs to varying degrees along the way from activity to travel outcome determines travel behavior, as expressed by which travel option is chosen. It is also consistent with the primary role of behavioral intention in other psychological theories derived from TRA, including TPB, TIB, TRB, IMB, and CADM. The hierarchy of travel needs can be thought of as related to the evaluation stage of NDM (Schwartz \& Howard, 1981), in which positive and negative attributes of the travel alternatives are weighed.

The hierarchy of travel needs is also consistent with (and was greatly influenced by) walking- and bicycling-specific travel behavior theories (Alfonzo, 2005; Schneider, 2013). The hierarchy of travel needs generalizes the hierarchy of walking needs (Alfonzo, 2005) including feasibility and accessibility as similarly described and a broader consideration of safety. It combines most of the aspects of comfort with pleasurability needs. The hierarchy of travel needs also borrows from the situational tradeoffs of the theory of routine mode choice decisions (Schneider, 2013). Awareness and availability 
are similar to the feasibility need, while safety/security and cost are as similarly described. Enjoyment related directly to the pleasure need.

That said, Alfonzo's (2005) conceptualization of a walking needs hierarchy differs slightly in its operation here. Congruent to Maslow's (1954) theory of motivation and "hierarchy of prepotency", Alfonzo suggested that higher-order needs in the hierarchy are typically not considered if lower-order, more basic needs are not met, while acknowledging that, in reality, that all lower-order needs need not be fully satisfied nor in this particular order.

This thesis takes an alternative view of the hierarchy of travel needs, one that is not limited by a sequential fulfillment of needs. In the hierarchy of travel needs, bundles of certain needs or even all needs may be considered simultaneously without the prior requirement to necessarily fulfill lower-order ones. The organization of the hierarchy merely reflects the hypothesized ranking of the influence of each factor group on travel decision-making in the general population. The exception is feasibility, which usually must be fulfilled for a travel alternative to be available. The other needs may act more as a utility function, where the value afforded by a travel option to the fulfillment of each travel need is weighted proportionally to the importance of that need.

One way to think about how the hierarchy of needs might operate is as a twostage process with both sequential and simultaneous considerations. First, questions define whether or not a travel option or alternative meets a certain threshold. Can I do it and does it fit in my schedule (feasibility)? Is the environment conducive to doing it (accessibility)? It is a safe and secure option (safety and security)? Alternatives that do not meet all three basic needs are rejected from consideration. Next, the time, monetary, 
and other costs of a travel alternative are compared to the many possible benefits (pleasure), and the most positive or least negative alternative is chosen. This possible decision-making rule can be summarized by one question: among the considered travel options that are feasible, accessible, safe, and secure, which one is best when considering the costs and benefits?

Another crucial consideration is that, from an economics, RUM, and discrete choice theory perspective, the hierarchy of travel needs might be thought of as comprising those observed and unobserved variables that make up a utility function. The feasibility need defines which alternatives comprise the consideration choice set. Costs like time and money are already common components of mode choice utility equations. Accessibility variables, including measures of density, diversity, and connectivity, are also common in discrete choice travel modeling. Some variables, including those to describe safety, security, and pleasure, are rarely observed and typically relegated to the utility function's error term; the theory of travel decision-making suggests they could be included, given that appropriate measures are constructed.

Note that these are just some possible ways for the hierarchy of travel needs to operate. Travel needs could act as screening rules to filter considered alternatives, outline a function of characteristics defining an alternative's utility, apply some other deliberative process, or use a combination of these methods. Also, the hierarchy may operate differently for different individuals or in different situations. The conceptual framework of the theory of travel decision-making is flexible enough to allow these different types of travel decision-making heuristics. 
Although the hierarchy of travel needs is depicted as a pyramid (à la Alfonzo, 2005), another shape may be more appropriate. For example, the hierarchy may be combined with the perception lens in an inverse pyramidal shape: $N$. Lower needs on the bottom, including feasibility and accessibility, might be more objectively and consistently evaluated, thus relying less on individual perceptions (although what is considered "feasible" may be very subjective). Alternatively, higher needs, such as pleasure, might be more subjectively defined, depending almost entirely on perceptions.

A final preliminary note on the hierarchy of travel needs is warranted. Within the theory of travel decision-making, the hierarchy of travel needs operates as the repository of objective measures to fulfill travel needs and afford travel. These lists include all of the variables that could possibly delineate the degree to which each need is met, and all of the attributes of alternatives that individuals might possibly consider. Later stages determine how decision-makers interpret and value this list or these categories of variables to fulfill certain travel needs.

The following sections describe the components of the hierarchy, including their hypothesized operation, examples of which factors falls into each, and empirical evidence to support the hypotheses.

\subsubsection{Feasibility}

\subsubsection{Definition}

Feasibility is the most basic need in the hierarchy of travel needs. When the choice of travel mode is the outcome, feasibility refers to the practicality or viability that 
making the trip via that travel mode is possible, considering individual or household constraints: mobility, schedule, time, other responsibilities, awareness, and availability of the travel mode. If an individual has limited mobility due to age, weight, or physical disability, some travel mode choices - namely, walk and bike - may not be feasible. Similarly, if a household does not own or have access to a motor vehicle and if they live in an area without fixed-route transit, then driving and riding transit are not feasible travel options for them to consider. Time and schedule also play a major role in travel decisionmaking; the constraints imposed by other prior scheduled activities and travel limit one's ability to travel to certain destinations and via certain modes.

Unique to this need is that without feasibility, no travel will take place. The other needs are neither necessary nor sufficient; feasibility is almost always necessary (but also not sufficient). Note that this restriction could be relaxed by assuming that some people (e.g., who may be consistently late) have misperceptions about what is truly feasible. In this case, there may be a minor threshold of infeasibility that may be allowed. Other people may not be aware of the feasibility of a travel option (e.g., riding a bicycle or taking transit).

The feasibility need could be viewed as delineating the complete choice set of alternatives that may be considered. When entering the hierarchy of travel needs within a travel decision-making process, potential travelers may think of a set or suite of possible travel options (e.g., destination, mode, etc.) that they are aware of and that meet their activity demand. They then - or perhaps simultaneously - evaluate the feasibility of each option with respect to the current situation. See the Decision Rule section for more information on means of filtering and selecting from among travel alternatives. 
The feasibility need is a basic part of the hierarchy of travel needs - and not a separate stage as in other theories (e.g., Schneider, 2013) - because, like other travel needs, it too relies partially on individual interpretation via the perception lens. A travel mode option considered feasible by one person may be perceived as infeasible by another person in the same situation, perhaps for reasons of self-efficacy (e.g., a lack of confidence about riding a bicycle) or awareness (e.g., a lack of knowledge about the existence of a transit line). Informational campaigns and other non-environmental interventions can result in changing perceptions of feasibility.

It is presumed that some prior travel-related decisions affect the feasibility of a travel option. In the short term, the scheduling of more important or skeletal activities (Akar, 2009) constrains the time available for other activities and for travel to them. In the longer term, decisions about residential location and vehicle ownership play major roles in the feasibility of different travel options, be they modes or destinations. Other social, economic, and especially life stage decisions also constrain the feasibility of certain travel options. Having children, caring for older adults, and working long hours or several jobs increase the complexity of travel and the infeasibility of certain travel options.

Although the feasibility need applies to all modes, it often has a different meaning for walking and bicycling. Whereas driving is (legally) limited to those with a driver's license and access to a motor vehicle, walking has no such barrier to entry (ignoring physical and medical limitations), and bicycling requires access to a vehicle but (usually, at least in the US) no license. On the other hand, individuals with visual impairments and other people with mobility devices can walk or otherwise roll but may be prohibited from 
operating a vehicle. Walking and cycling are often slower modes of transportation, meaning they may be less feasible when schedules are tight.

The need for a feasible travel option has found theoretical support. Hägerstrand (1970) proposed capability, coupling, and authority constraints which limited feasible activity and travel options. Alfonzo (2005) labels this need "feasibility" and defines it in a similar way as in this theory, placing it at the most basic level in the hierarchy of walking needs. Feasibility also appears in Schneider (2013) as the "availability" component of the first step. In the theory of routine mode choice decisions, availability has a similar choice-set-defining role to feasibility here, in that tradeoffs are only made between modes that are available.

\subsubsection{Examples and Empirical Support}

Components of the feasibility need include individual, household, and intrahousehold characteristics but explicitly exclude components of the environment as they are captured by other travel needs. Socioeconomic and life stage characteristics have a large role in defining feasible travel. Examples of factors that influence feasibility individual mobility, mode availability, activity and travel schedules, and household interactions and other responsibilities - are described in more detail in the following sections.

\subsubsection{2.a. Individual Mobility}

Characteristics that define an individual's mobility and influence the feasibility of travel options include age, weight, health, physical disability, cognitive disability, or 
other mobility and decision-making limitations. These individual mobility factors are especially important for evaluating the feasibility of travel mode decisions that involve walking or bicycling. Those who are overweight may be less able to walk or cycle as far. Young children and older adults or those with certain health problems or physical disabilities may be more limited with respect to active travel than for using transit or being a passenger in a car. People with certain mobility disabilities may require another person to escort them as they travel - a coupling constraint (Hägerstrand, 1970) - thus reducing the feasibility of travel options for both parties.

Individual mobility factors, including physical abilities and age, have been found to be important influences on walking, cycling, and general physical activity. Older adults have been found to have a greater difficulty crossing streets, in part because of slower walking speeds or visual impairments (Langlois et al., 1997). Visual impairment has been strongly associated with difficulty walking (Crews \& Campbell, 2001). Obesity can be a perceived barrier to physical activity (Ball et al., 2000). Injuries and disabilities are also used as justifications for the infeasibility of physical activity (Ball et al., 2000; Finch, Owen, \& Price, 2001).

Skill is another factor defining the feasibility of travel options. Acting as a capability constraint (Hägerstrand, 1970), skill - the ability to act or perform a behavior and associated knowledge are necessary for some travel options. One must learn how to walk and ride a bicycle, be trained and licensed on how to operate a motor vehicle, and have the ability to navigate a transit system. Others lose skills over time through aging, such as the ability to react to obstacles or safely drive at night. 


\subsubsection{2.b. Awareness and Availability}

Key characteristics that also affect the feasibility of travel options are the availability (and awareness) of different travel modes. Clearly, a necessary condition for traveling in a vehicle is the availability of that vehicle type. Owning an automobile, subscribing to a car-share program, or having a motor vehicle available for use is necessary for making a driving or passenger travel option feasible. Living or working near transit stops/stations or being able to call a demand-responsive transit vehicle is necessary for making a transit travel option feasible. Owning a bicycle, being able to access a bicycle-share program, or otherwise having a bicycle available for use is necessary for making a bicycle travel option feasible. Walking travel options are feasible for all, assuming other mobility or schedule constraints are not limiting.

Mode availability also plays a role in shorter-term travel decisions, such as triplevel decisions while on a tour. For the most part, someone who has driven their car or ridden their bicycle from home to one activity location must take their (motorized or nonmotorized) vehicle with them when they travel to their next destination or when they return home. People who walk from their home may have more flexibility in later modal travel decisions (they may walk or take transit), or they may be constrained by not having access to a vehicle that was left at home. Another important aspect of mode availability is the scheduling of the use of shared vehicles. In a household with more drivers than autos, if all cars are in use then driving mode options are not feasible for another driver. Also, having a driver's license is a prerequisite for feasibly being a driver, although some may violate this legal requirement. 
It is important to note that the influence of mode availability on defining the feasibility of travel options may be weakening as more travel technologies emerge in the marketplace. Carsharing programs like Zipcar, Car2Go, and Getaround make owning a motor vehicle less important for driving travel options and enable one-way auto trips in certain urban areas. Ridesharing services and companies like Sidecar or casual carpooling (slugging) may offer an opportunity to make impromptu auto passenger travel a feasible option for some travelers. Municipal and private bicycle-sharing systems around the world have been shown to dramatically increase the number of travel and activity opportunities that are now feasible. These developments increase both the feasibility of a number of new travel options and the difficulty of predicting and modeling them.

Modal availability has long been a key variable in determining travel decisions, particularly mode choice. Car or automobile ownership has a significant influence on the choice of travel mode; increasing car ownership is associated with an increasing likelihood of driving (Cervero \& Kockelman, 1997; Quarmby, 1967) and a decreasing likelihood of walking or cycling (Dieleman, Djist, \& Burghouwt, 2002; Plaut, 2005). Some authors have noted that vehicle ownership is not necessarily an exogenous choice, but one that is related to the built environment and travel decisions (Bhat \& Guo, 2007; Cao, Mokhtarian, \& Handy, 2007; Pinjari et al., 2011; Train, 1980; Van Acker \& Witlox, 2010). A similar positive relationship has often been found between bicycle ownership and cycling (Handy et al., 2010). Proximity to transit stations, stops, and/or service has often been found to have a positive effect on pedestrian activity or mode choice (Schneider, Arnold, \& Ragland, 2009). 
It is also important to note that the role of mode availability on travel behavior may be strongly determined by perceptions of mode availability. A lack of awareness (perceived availability) that walking and bicycling are options may deter the use of active modes. Studies have shown that perceptions of walking time are frequently inaccurate, especially beyond close destinations (Horning, El-Geneidy, \& Krizek, 2008), possibly leading to overestimates of walking time and lack of awareness that walking may fit within one's schedule. Many travelers are not aware of the full range of transit mode alternatives available to them (Outwater et al., 2011).

Awareness extents to other non-mode travel contexts as well. The primary example of this is awareness of destinations, especially for someone in an unfamiliar area. Information systems, including detailed interactive internet-based maps accessible through mobile devices, are raising the awareness of possible destinations to fulfill a generic activity demand such as grocery shopping or eating out. These awareness considerations are also discussed within the section on perceptions as mediators.

\subsubsection{2.c. Activity and Travel Schedules}

Among the most important characteristics to define the feasibility of travel options are the scheduling of other activities and travel and the flexibility of that scheduling. Some activities may be highly fixed in time and space: school and often work. Other activities planned in advance can also be considered fixed: things like medical appointments, social engagements, concerts and shows, or vacations. These skeletal activities impose temporal and spatial constraints both on travel to and from them and on the scheduling of other more discretionary activities and travel. A working mother 
may have to finish her shift at a set time and pick up her son from day care half-an-hour later; based on that time constraint and locations of the two activities, her only feasible travel mode option is to drive. A person who is retired with no other daily obligations likely has a greater number of feasible travel options to buy groceries than does a working person with child-rearing and volunteering obligations.

Since the introduction of the time-space prism by Hägerstrand (1970), one of the biggest advancements in travel behavior research has been including the effects of activity scheduling on travel. Many activity-based model systems have been proposed and some have been constructed and implemented (Axhausen \& Garling, 1992; Ettema \& Timmermans, 1997; Timmermans, 2005). One key component is the scheduling of activities, around which travel can (and often need) occur. Usually some activities are first assumed to be fixed or mandatory, while other discretionary activities may vary in time. The availability of travel modes may depend on the flexibility of these activity schedules. Some research has begun to redefine activity types in order to more realistically represent the activity-travel choice process (Akar, 2009; Doherty, Miller, Axhausen, \& Gärling, 2001).

Several studies have found that personal schedules and time constraints affect travelers' choices of modes. One study found perceptions that walking to school was convenient significantly increased the odds of children walking to school, above and beyond the impact of distance (Trapp et al., 2012). An analysis of perceptions about walking suggested personal characteristics - time or physical limitations - were stronger reasons for not walking than environmental characteristics like too much traffic or a lack of sidewalks (Handy, 1996). The availability of time for travel between scheduled 
activities is of particular importance to walking and cycling as these are typically slower travel modes.

\subsubsection{2.d. Household Interactions and Responsibilities}

Other socioeconomic characteristics and one's stage in life can also inform the feasibility of travel options. The most notable of these are inter-personal, intra-household, and inter-household interactions and responsibilities. A person's feasible travel options are defined not only by his or her own mobility characteristics, modal availability, and skeletal activity schedule, but also those activities that she or he needs to conduct jointly with other household members or persons: Hägerstrand's (1970) coupling constraints. Responsibilities include escorting children to activities, parents and grandparents to medical appointments, and friends to the airport. Activities and travel conducted jointly are often much less flexible than individual travel because two or more people must reconcile their differing needs and perceptions. Thus, certain travel options may not be feasible when joint travel is considered. For example, if a working couple wants to commute home together but one partner does not have access to or want to ride a bicycle, then it is infeasible for the other partner to commute by bicycle.

A growing body of literature has emerged examining the effect of intra-household interactions on activity and travel decisions. Most of this work focuses on modeling joint choices of activities or activity patterns with the goal of integrating findings into emerging tour- and activity-based travel demand modeling efforts (Gliebe \& Koppelman, 2005; Vovsha, Peterson, \& Donnelly, 2005). In general, intra-household activity research points to the importance of factors like working hours and schedules, parental gender, 
auto availability, and the presence of (young) children (Gliebe \& Koppelman, 2002; Schwanen, Ettema, \& Timmermans, 2007; Srinivasan \& Bhat, 2005).

Some work has found influences of intra-household interactions and responsibilities on restricting or facilitating the feasibility of walking and bicycling. Work commitments have been shown to decrease the likelihood of walking or obtaining sufficient weekly physical activity (Salmon, Owen, Crawford, Bauman, \& Sallis, 2003). The frequency of participating in physical activity with friends or family was positively associated with being adequately active (Booth et al., 2000). Parental factors have also been found to significantly impact children's travel mode to/from school. In part because women were still more likely to escort or chauffer children to and from school (Schwanen, 2007), children of working mothers were less likely to walk or cycle (McDonald, 2008; Yarlagadda \& Srinivasan, 2008).

\subsubsection{Accessibility}

\subsubsection{Definition}

Accessibility refers to the feasibility of the travel option based on the constraints of the built and natural environments, and includes as factors the locations of key activities, the number of possible destinations, connectivity and barriers, and other physical path-based characteristics. Accessibility could be considered to have two components: one that defines the feasibility of travel based on constraints of the built/natural environment, and one that affects the quality or number of options for travel in the built environment. Whereas the feasibility need defined whether or not a travel 
option was feasible to the individual, based on his or her mobility, schedule, and the mode, accessibility feasibility defines whether the built/natural environment is conducive for such travel. For example, the proposed activity destination must be close enough to the current location in order to travel within the feasible time window. The other aspect of accessibility defines the environmental aspects that contribute to the quality of the travel option. For example, for walking to be an attractive option, there should at least be a walking path or sidewalk connecting two activity locations.

Accessibility and feasibility can work in tandem to define what travel options are feasible in space and time. Feasibility captures the individual aspects, and accessibility captures the environmental aspects. The distinction is not clean and both are usually simultaneously considered. For example, when choosing destinations, people consider not only whether there is enough time to get there but also how far they are from one another. Accessibility and the environment (the transportation system) supplies the distances that are later used to calculate cost attributes. Accessibility can also inform an assessment of the resilience of a particular travel option. Travel options with higher accessibility may have more opportunities to deviate from the chosen one if situations change. For example, choosing to walk to a store in a diverse local neighborhood business district may be a more resilient option than driving to an isolated specialty big box store in the suburbs if the traveler realizes she needs other goods while out. In these ways, aspects of accessibility may overlap with the cost need, and certain factors may partially contribute to both needs.

Accessibility likely affects walking and bicycling more than non-active travel modes. The characteristics of walking and cycling - their exposed nature, the slower 
speeds at which they take place, and their operation under human power - mean that the environment (in a broad sense) exerts a stronger influence on these active modes of transportation.

The need for accessibility appears in several theories of travel behavior. Alfonzo (2005) labels this need "accessibility" and defines it in a similar way, placing it immediately above feasibility in the hierarchy of walking needs. Accessibility also appears in Schneider (2013) as the strongest factor influencing walking and bicycling in the "cost" component of the third step. In the theory of travel decision-making, accessibility will be defined as primarily a function of two components (Tal \& Handy, 2012): 1) proximity between residential, job, and school locations and to other destinations; and 2) connectivity or measures of network connections, barriers, and paths.

\subsubsection{Examples and Empirical Support}

A number of reviews have investigated the impact of land use, urban form, or the built environment on travel behavior and/or physical activity (e.g., Badoe \& Miller, 2000; Badland \& Schofield, 2005; Crane, 2000; Ewing \& Cervero, 2001, 2010; McCormack et al., 2004; Saelens, Sallis, \& Frank, 2003; Saelens \& Handy, 2008). Early reviews highlighted how most research had focused on relationships between the environment and automobile or transit use; few studies had looked at walking and almost none at influences on bicycling (Badoe \& Miller, 2000; Crane, 2000; Ewing \& Cervero, 2001). Later reviews noted an increased focus on walking and bicycling (Ewing \& Cervero, 2010) or specifically investigated relationships between the built environment and physical activity or these active travel modes (Badland \& Schofield, 2005; McCormack 
et al., 2004; Saelens, Sallis, \& Frank, 2003; Saelens \& Handy, 2008). Still, few studies have looked at bicycle effects in particular (Ewing \& Cervero, 2010).

Most studies still focus on the role of what can be called environmental accessibility factors. The accessibility need captures those environmental aspects of travel explicitly excluded from the feasibility need. Again, accessibility can be thought of as defining both the feasibility and the quality of the travel (built and natural) environment. Examples of factors that influence accessibility - residential, job, and school locations; proximity of destinations; diversity of destinations; connectivity and barriers; and physical path-based characteristics - are described in more detail in the following sections.

\subsubsection{2.a. Residential, Job, and School Locations}

The chosen (or assigned) locations of a person's home, work, and/or education activities are primary drivers of accessibility simply because most time is spent at activities in these locations and because these activities are more likely to be fixed in time (and space). The relative location and associated distances between home, work, and school determine accessibility and thus assessments of travel options.

Travel mode choice studies have long found distance to be an overriding factor for work and school trips, even when they began considering walking and bicycling. An early review noted how trip distance was the biggest determinant of walk mode choice (Badoe \& Miller, 2000). A British study of young students' travel modes to school found that walking dropped precipitously for those who lived more than a mile away (Black, Collins, and Snell, 2001). One study found significant linear and quadratic relationships 
between distance to school and children walking (Trapp et al., 2012), suggesting that the siting of schools in relation to homes can impose limitations. See the subsection on Travel Time within the Cost need section for additional related research findings.

\subsubsection{2.b. Proximity of Destinations}

In addition to a person's home, work, and school locations, the nearby location of other destinations also influence accessibility. A greater number of uses in closer proximity to one's home, work, or school increases accessibility, or the number of opportunities (travel options). It may also increase resilience. In the literature, this characteristic of accessibility has usually been called or operationalized as proximity, distance, or density.

The most consistent finding of one meta-review is that proximity to nonresidential destinations was associated with increased walking (Saelens \& Handy, 2008). Of particular importance may be proximity to shopping and transit. Shorter distances to the nearest store and transit stop have been positively related to walking (Ewing \& Cervero, 2010), while increased distance to commercial land uses was associated with a decreased likelihood of walking for non-work trips (Reilly \& Landis, 2003). Greater distances from home to routine destinations like restaurants, grocery stores, recreational facilities, and transit stops/stations were associated with decreased odds of walking for transportation (McConville, Rodríguez, Clifton, Cho, \& Fleischhacker, 2011). Perceived accessibility and proximity to destinations have also been positively associated with physical activity (McCormack et al., 2004). 
Reviews also consistently find positive associations between density - especially residential density, employment density, and commercial floor area ratio - and walking (Ewing \& Cervero, 2010; Saelens \& Handy, 2008). An intensity or density factor was positively related to the likelihood of traveling by anything but a personal vehicle for non-work trips (Cervero \& Kockelman, 1997). Increased population density was associated with an increased likelihood of walking for non-work trips (Reilly \& Landis, 2003). Retail floor area ratio was positively related to transportation walking (Saelens, Sallis, Frank, et al., 2012).

Density's effects may differ based on travel context. Employment density for nonhome trip ends was as or more important than population density at the home end for walk mode choice (Ewing \& Cervero, 2001). Residents of areas with high population density had twice the odds of walking for transportation as did residents of low density areas, but density had no effect on the odds of leisure walking or on total walking or physical activity levels (Oakes, Forsyth, \& Schmitz, 2007).

Local density appears to matter more than regional density. Population density at the Census block group level, but not at the zip code level, was positively related to nonwork walk trip frequency (Greenwald \& Boarnet, 2001). Greater local intensities of offices, retail stores, grocery stores, and bus stops were associated with increased odds of walking for transportation (McConville et al., 2011).

\subsubsection{2.c. Diversity of Destinations}

In addition to a person's home, work, and school locations, the location of other types of destinations also influence accessibility. A greater variety of uses in closer 
proximity to one's home, work, or school increases diversity, or the number of different opportunities (unique travel options). It may also increase resilience. There have been many different ways to measure diversity in the literature, but they all attempt to get at the mixing of land-uses.

Reviews consistently find positive associations between diversity measures including land use mix and jobs-housing balance - and increased use of active travel modes (Badland \& Schofield, 2005; Ewing \& Cervero, 2001, 2010; Saelens, Sallis, \& Frank, 2003; Saelens \& Handy, 2008). Individual studies also suggest that land use diversity is particularly important for walking. Land use mix was positively associated with the odds of making a non-SOV non-work trip (Cervero \& Kockelman, 1997). Decreased proportions of detached residential housing were associated with increased odds of walking for non-work trips (Reilly \& Landis, 2003). A comparison of conventional and neo-traditional neighborhoods found walking trips substituted for driving in the neighborhood with mixed uses (Khattak \& Rodriguez, 2005). A greater number of different local land use types was associated with an increased odds of walking for transportation (McConville, et al., 2011).

\subsubsection{2.d. Connectivity and Barriers}

Other aspects of the built environment influence accessibility, notably connectivity and barriers. These factors influence the ease of getting around by various travel modes. Like other aspects of urban form (Clifton, Ewing, Knaap, \& Song, 2008), connectivity has been measured in a variety of ways: block length, block size, block density, intersection density, connected intersection ratio, link-to-node ratio, percentage 
of four-way intersections, percentage of grid-like blocks, and route directness (Dill, 2004). Other factors and measures include terrain, natural barriers like hills and water features, and built barriers like highways and buildings. Measures specific to walking and bicycling include sidewalk availability and connectivity and the type, extent, and connectivity of bicycle facilities. Whether or not facilities exist to facilitate walking and bicycling are key considerations in determining whether to use those travel modes. In addition, walking typically involves multiple routes that are similarly fast, so higher connectivity provides more route options while walking, which may be useful for people who prefer variety. Several reviews have concluded that sidewalk and street connectivity measures - including sidewalk continuity and intersection density - are positively correlated with walking and cycling (Ewing \& Cervero, 2010; Saelens \& Handy, 2008; Saelens, Sallis, \& Frank, 2003).

Neighborhood comparison studies frequently find that walkable neighborhoods those with greater connectivity and fewer barriers - see higher levels of walking. Pedestrian volumes in urban neighborhoods with small blocks and continuous sidewalks were three times as high as in suburban neighborhoods (Hess, Moudon, Snyder, \& Stanilov, 1999). Residents of high-walkability neighborhoods made more walking trips, particularly for running errands, than did residents of low-walkability neighborhoods (Saelens, Sallis, \& Frank, 2003). A comparison of conventional and neo-traditional neighborhoods found walking trips substituted for driving in the neighborhood with smaller block sizes and increased connectivity (Khattak \& Rodriguez, 2005).

Connectivity factors have been shown to play a facilitating role in decisions surrounding walking and bicycling in a number of studies as well. A measure of 
connected intersections was positively associated with the odds of making a non-SOV non-work trip (Cervero \& Kockelman, 1997). Continuous sidewalks between one's origin and destination were associated with higher odds of walking to access transit or walking to a college campus (Rodríguez \& Joo, 2005). A factor of nine different street connectivity measures, representing shorter connected blocks, positively and significantly influenced the propensity and duration of active transportation (Berrigan, Pickel, \& Dill, 2010). Pedestrian network connectivity contributed to the attractiveness of walking environments (Adkins, Dill, Luhr, \& Neal, 2012).

Occasionally, research finds that the effect of connectivity on active travel is not significant or applies only to certain people or conditions. In one study, connectivity measures were not associated with walking behavior (Reilly \& Landis, 2003). In another, street connectivity, as measured by block size, did not influence the odds of walking for travel or leisure (Oakes et al., 2007). The perceived availability of sidewalks was associated with an increase in walking duration for men only (De Bourdeaughuij, Sallis, \& Saelens, 2003). One study found that high connectivity was related to increased odds of children walking to school, but only for boys and only when traffic volumes were low (Trapp et al., 2012). These findings highlight the need to better understand these relationships.

\subsubsection{2.e. Physical Path-Based Characteristics}

In addition to connectivity and barriers, other path-based environmental characteristics influence accessibility. Characteristics of the built and natural environments along paths of travel are arguably more important for walking and 
bicycling than for riding transit or driving. These active travel modes are exposed to the elements rather than trapped within glass and are thus more able to observe and interact with the surrounding environment. Path-based environmental measures include the completeness, surface type, and quality of sidewalks and pedestrian paths, or the type, maintenance, and quality of bicycle facilities. Grade also plays a directional path-based role in defining accessibility, although it also informs physical effort exerted, a component of the cost need. Some (Forsyth \& Krizek, 2011) argue that urban design should be tailored differently for cycling, walking, and driving, and that it should pay more attention to the experience of using a facility.

Although most early research on the travel behavior effects of the built environment measured at trip origins and destinations only, more recent efforts have found significant effects of path-based environmental characteristics on walking and cycling. Children who passed an engineering improvement to the sidewalk, crossing, or traffic control on their way to school were more likely to show increased walking and bicycling than children who did not pass the Safe Routes to School project (Boarnet, Anderson, Day, McMillan, \& Alfonzo, 2005). One study found that the proportion of parking lots along an individual's travel corridor was negatively associated with the likelihood of walking and bicycling to access rapid rail transit stations (Appleyard, 2012). Path-based measures of employment density, roadway capacity, grade, and route directness were found to be positively associated with walking tour mode choice in a recent model estimation study (Bomberg, Zorn, \& Sall, 2013). Pedestrian and cyclist route choice research also reveals the importance of accessibility characteristics along the travel path, including sidewalks that are in good condition (Agrawal, Schlossberg, \& 
Irvin, 2008), types of bicycle facilities, traffic control devices, traffic volumes (Broach et al., 2012), numbers of turns, slopes of roadways (Hood et al., 2011), and types of adjacent on-street parking (Sener, Eluru, \& Bhat, 2009).

\subsubsection{Safety and Security}

\subsubsection{Description}

The need for safety and security during travel is derived directly from Schneider (2013). People make travel decisions to provide them a basic level of: 1) safety, from traffic collisions; and 2) security, from crime. Alfonzo (2005) had a similar "safety" need, but one that is wholly determined by an acceptable level of safety from crime. In the hierarchy of walking needs, traffic safety is placed within a "comfort" need because, for walking, Alfonzo presumed concerns about crime take precedence over concerns about safety while noting that this hierarchy may be reversed or simultaneously considered for

some people. Safety and security are prime examples that justify the need for a perception lens that mediates travel needs; objective measures of safety and security likely matter less than the subjective assessments of them.

Safety and security needs are thought to be especially relevant for travel options involving walking and bicycling. A need to feel safe from traffic collisions may be stronger for bicycling because cycling often takes place in or adjacent to automobile traffic lanes, whereas walking mostly occurs on dedicated pedestrian facilities: sidewalks. Conversely, a need to feel secure from possible crime may be stronger for walking because one cannot escape a dangerous situation as easily on foot as on a bike or in a 
motor vehicle. A Delphi study of experts suggested that traffic safety was a more important consideration than personal security for cycling, whereas both were important for walking (Pikora et al., 2003). However, both safety and security are likely concerns to some extent for both walking and bicycling. People walking may be concerned about collisions with motor vehicles along arterials/highways without sidewalks, at long and busy street crosswalks, when crossing many driveways, or even on crowded multi-use paths and trails. People bicycling may be concerned for their personal security at night or in certain neighborhoods with perceived crime problems. Others may be afraid of having their bicycles stolen.

Although safety and security are thought to be concerns primarily around walking and bicycling, this need does have a role in other travel situations. For instance, some people may be afraid to drive through or park in various neighborhoods at certain times of day, affecting their decisions of destination and route. Other people may avoid a particular stretch of road because it has a high concentration of speeding or impaired drivers. Safety also comes into play when deciding how or whether to travel during adverse weather conditions. Slick or icy roadways, sidewalks, or bus stops may deter certain travel options.

The safety and security need suggests that people will only consider travel options that provide them a minimum of protection from traffic and crime. In the framework of travel decision-making, this need may be a binary evaluation: yes, I feel safe and secure; or no, I do not feel safe and secure. A threshold level of safety from traffic and security from potential crime, dependent on individual perceptions, must be achieved if the travel option is to be chosen. Mathematically, this could involve a set of safety/security 
variables that, if it fails to meet a perceived threshold value when linearly combined according to an individual's weighting scheme, triggers a large negative coefficient in the travel option's utility equation. An alternative hypothesis is that travelers may consider safety and security as attributes or components of utility to be maximized alongside other characteristics of travel alternatives.

\subsubsection{Examples and Empirical Support}

Examples of factors that influence the safety from traffic and security from crime needs are described in more detail in the following sections. These factors are similar to the safety factors and distinctions noted by Pikora et al. (2003).

\subsubsection{2.a. $\quad$ Safety from Traffic}

Many characteristics of the built environment may influence traffic safety and perceptions thereof, particularly while walking or bicycling (Ewing \& Dumbaugh, 2009). Traffic volumes and traffic speeds are two common features that directly influence safety. For bicycling, lower-volume streets and dedicated or separated bicycling facilities (ranging from signs and sharrows to bicycle boulevards and off-street trails) may increase perceived and actual levels of safety. The presence of other traffic calming features may increase perceived and actual safety for both bicycling and walking (Pucher \& Dijkstra, 2003). Urban form characteristics, such as the length of blocks, extent and quality of sidewalks, and the number of driveways may impact perceived safety for potential pedestrians. Design features that provide buffers between vehicles and people walking tend to increase perceived safety. Street crossing characteristics, such as signalization, 
signing, and striping, as well as visibility and lighting, likely play a large role in how people perceive the safety of walking. Even the overall amount of walking and bicycling in a community may affect the number of collisions experienced by people walking and cycling (Jacobsen, 2003).

Concerns over traffic safety may dominate some travel decisions, such as route choice or children's travel to school, but be less influential in other travel decision contexts, such as destination or mode choice. In fact, the record is mixed on the significance of traffic safety on walking and bicycling. In one study (Handy, 1996), the perception that it was safe to walk in the neighborhood was the strongest correlate of walking frequency for strolling trips, but was the second biggest factors after distance for trips to the store. Conversely, crossing or walking along busy streets was negatively correlated with walking to local commercial areas (Handy, 1996). Although perceptions of heavy or bothersome traffic were positively associated with walking (Brownson, Baker, Housemann, Brennen, \& Bacak, 2001), it may be that those who walk the most are more aware of traffic conditions in their neighborhood (McCormack et al., 2004). Perceived safety from traffic was not associated with a change in walking duration (De Bourdeaughuij et al., 2003). Residents of high-walkability neighborhoods had more positive perceptions of traffic safety than did residents of low-walkability neighborhoods (Saelens, Sallis, Black, \& Chen, 2003). Perceived neighborhood traffic safety levels were associated with walking for pleasure but not for other purposes (Humpel, Owen, Iverson, et al., 2004). Despite significantly greater concerns among women about traffic safety and auto speed in particular, while walking in their neighborhoods, models of distance walked and number of days walked showed no significant effects of perceived traffic 
safety (Clifton \& Livi, 2005). Perceptions about the volume and speed of traffic were not related to walking duration (Rodríguez, Aytur, Forsyth, Oakes, \& Clifton, 2008). Arterial streets were perceived to be less attractive for walking, while sidewalks segments set back from the curb were more attractive (Adkins, et al., 2012). Although most studies on traffic safety were conducted in developed countries in Europe, North America, and Australia, inconclusive results have also been reported in developing countries like Brazil (Parra et al., 2011) and Nigeria (Oyeyemi, Adegoke, Sallis, Oyeyemi, \& De Bourdeaudhuij, 2012).

In contrast, research on children's travel to school finds more consistently that concerns over traffic safety impact the choice to walk or bike. One study found an overwhelming percentage of parents $(80 \%)$ were concerned about road safety, and that negative perceptions about the safety of crossing streets were negatively associated with frequency of 10-to-12-year-olds walking and cycling to school (Timperio et al., 2004). Low traffic volumes and higher perceptions of safe traffic crossings increased the odds of children walking to school (Trapp et al., 2012). Parental concerns over traffic volume, speed, and intersection safety were the second most important issues, after distance, affecting children's choice of travel mode to school. These factors were also second in importance, after crime, for households living within a mile of school (Zhou, Yang, Hsu, \& Chen, 2010). Student perception of parental perceptions of safety was related to a decrease in odds of children aged 6 to 10 walking to school and to the park (Broach \& Dill, 2013).

Research on pedestrian and cyclist route choice also reveals environmental characteristics that frequently shape perceptions of traffic safety and thus influence 
walking and bicycling. People walking to access rail transit reported concerns over safety from traffic were second only to distance or speed in factors influencing their chosen route. About $85 \%$ of respondents rated having traffic devices present and safe speeds of traffic as very or somewhat important, second only to shortest route (Agrawal et al., 2008). A revealed-preference survey and route choice model of routine bicycle users suggested a strong preference for routes that reduced exposure to motor vehicles. The model indicated preferences for protected bicycle paths and facilities with lower traffic volumes, like bicycle boulevards, and suggested cyclists were willing to travel up to 40$60 \%$ longer distances to avoid turning left or crossing streets with greater than 20,000 ADT at unsignalized intersections (Broach et al., 2012). These findings mirror those of other studies that suggest people walking and bicycling consider traffic safety when choosing a route (Hood et al., 2011; Sener et al., 2009; Westerdijk, 1990; Winters, Davidson, Kao, \& Teschke, 2011).

\subsubsection{2.b. Security from Crime}

A number of environmental characteristics may be interpreted by different people to suggest concerns for personal safety, including urban form characteristics, specific land use types, and the absence of people or the presence of certain individuals or groups of people (Alfonzo, 2005; Loukaitou-Sideris, 2006, Loukaitou-Sideris \& Eck, 2007). The presence of things like graffiti, vandalism, trash, broken windows, or abandoned buildings/lots may increase fear of crime and deter walking and/or bicycling (Foster, Giles-Corti, \& Knuiman, 2011). Urban design characteristics like narrow streets, unobstructed views, first floor windows, street lights, decorations, plantings, and the 
presence of stores may reduce perceived crime levels and facilitate more walking. Pawn shops, bars, and liquor stores may be examples of land uses that some potential pedestrians may avoid walking past. Regular activity, signs of activity, or eyes on the street may yield lower levels of perceived crime. The presence of certain groups of people - young males, for example - may increase levels of perceived crime. Research on environmental preferences finds that enclosed urban spaces with limited opportunity for escape (e.g., alleys) are associated with higher fear of crime. In one study, although urban nature and urban alley scenes were rated as mysterious, a positive preference factor, the higher fear of danger from humans in alleys depressed alley preference scores (Herzog \& Smith, 1988). When analyzing evaluation of computer generated environmental scenes, impressions of safety were influenced more by locomotive permeability than by visual permeability (Stamps, 2005).

Limited empirical evidence supports the theory's hypothesis that a concern over crime security or victimization can be a significant factor in walking decisions, although it is less important than other needs like accessibility, traffic safety, or time. Fear of burglary or being victimized has been negatively related to number of days walked (Ross, 2000). Perceptions of safety have been positively associated with physical activity (McCormack et al., 2004), where perceptions of high crime or a lack of safe places were related to a decreased odds of physical activity (Brownson et al., 2001). In one study, higher rates of property and violent crime were associated with a decreased likelihood of walking to work but not for non-work trips, and population density and accessibility were stronger factors than personal security (Ferrell, Mathur, \& Mendoza, 2008). Another study found significant concerns about crime at transit stations among females, 
specifically that women using high crime light-rail stations or traveling at night were more likely to be picked-up than to drive and park, ride the bus, or walk (Kim et al., 2007). Parental concern over violence and crime was an important factor affecting children's choice of travel mode to school, particularly for households living within walking/bicycling distance of school (Zhou et al., 2010). Although most studies on the impact of safety from crime on walking and bicycling were conducted in developed countries in Europe, North America, and Australia, significant impacts have also been reported in developing countries like Brazil (Parra et al., 2011) and Nigeria (Oyeyemi et al., 2012).

Other studies find no significant effects of security concerns after controlling for other factors. Perceived safety from traffic was not associated with a change in walking duration (De Bourdeaughuij et al., 2003). Perceptions of crime safety did not differ between residents of high- and low-walkability neighborhoods (Saelens, Sallis, Black, \& Chen, 2003). Despite significantly greater concerns among women about personal security while walking in their neighborhoods, models of distance walked and number of days walked showed no significant effects of perceived safety from crime for men or women (Clifton \& Livi, 2005). Similarly, despite overwhelming parental concern (80\%) about stranger danger, these concerns were not significantly related to children's frequency of walking or cycling to school (Timperio et al., 2004). In a study of pedestrian routes to access rail transit, only a few respondents mentioned that crime concerns affected their choice of route (Agrawal et al., 2008). These findings may be confounded by issues of self-selection, either by people with lower tolerances for crime choosing to reside in low-crime neighborhoods, or crime-fearing individuals choosing to use other 
travel modes. Alternatively, observed travel behaviors may have depended more on other factors than on perceptions of crime.

Less evidence points to personal security playing a role in bicycling decisionmaking. A Delphi study of experts suggested that personal security was less important than traffic safety for cycling (Pikora et al., 2003). Property crime rates were not associated with a change in likelihood of bicycle trips for work and non-work purposes (Ferrell et al., 2008). A stated-preference survey of deterrents to cycling found the risk of violent crime was less important than auto collision risks (Winters et al., 2011). A revealed-preference study of cyclist route found no impact of the number of violent crime on choice of route (Hood et al., 2011).

\subsubsection{Cost}

\subsubsection{Definition}

The cost need reflects the time, money, and effort expended on the travel option. More specifically, these costs include the costs of travel time, monetary costs, physical effort or energy exerted, mental effort exerted, and the possible costs of inconvenience or unreliability. Any opportunity costs associated with choosing one travel option over another are also included. Costs reflect all of the negative aspects of travel that are to be minimized when making a rational choice. Statistically, cost is a disutility; all of the variables influencing cost should have negative coefficients in the modeled equation.

Theory and practice provide support for the inclusion of a desire to reduce costs while traveling. Surprisingly, Alfonzo (2005) did not include cost anywhere in the 
hierarchy of walking needs. In contrast, cost and convenience are key constructs in the theory of routine mode choice decisions (Schneider, 2013). Among the important cost considerations were time, money, physical effort, cognitive effort, and convenience. In practice, cost may be operationalized as a generalized cost function with contributions from all of these components.

\subsubsection{Examples and Empirical Support}

Examples of factors that influence what can be defined as cost - time, monetary cost, physical effort, mental effort, and convenience and resilience - are described in more detail in the following sections.

\subsubsection{2.a. $\quad$ Time}

The most direct cost of travel that is considered during the travel decision-making process is time. Which travel option will get me to my desired activity destination in the fastest way possible? Travel time will depend on characteristics of the travel option under consideration: route, destination, mode, time-of-day, etc. Riding transit between two locations may be faster than bicycling during most of the day, but bicycling may take less time during peak periods. Walking anywhere typically takes more time than traveling by any other mode, but for some short trips it may be faster to walk when factoring in congestion, access, egress, and waiting time (for transit) or parking time (for driving and bicycling). In addition, the value or impact of time may vary depending on the travel mode or even the component of a travel option or sequence. Commonly, travelers by transit tend to place a higher value on waiting and transfer time than they do on in-vehicle 
travel time, meaning five minutes of waiting time is more costly than five minutes of time sitting on the vehicle. Similarly, people may be more willing to spend five minutes driving around in search of the best parking spot rather than spending those five minutes walking from a more distant location. Finally, individuals may have different values of time based on individual socioeconomic characteristics or by time-of-day or destination; this is why the perception lens mediates the impact of objectively-measured time on travel decision-making.

A simple distance- or time-based measure of level-of-service has long been a major factor in the choice of travel mode ever since walking and bicycling began to be included in mode choice studies. Early mode choice models in regional travel demand forecasting model systems simply included trip distance as the primary if not only variable in utility equations for walk, bicycle, and non-motorized modes (Cambridge Systematics \& Barton Aschman Associates, 1994; Cambridge Systematics, Parsons Brinkerhoff Quade \& Douglas, \& S. H. Putman Associates, 1996; Purvis, 1997; Rossi, 2000; Singleton \& Clifton, 2013). Even today, every mode choice model used by large metropolitan planning organizations (MPOs) that considers walking and bicycling includes travel time or trip distance to predict the share of trips by active travel modes (Singleton \& Clifton, 2013).

Recent academic research on disaggregate individual choice data highlights the primacy of travel time and distance for walking and cycling mode choice in all kinds of situations. A study of travel mode choice to access rapid rail transit stations found that distance was strongly and negatively associated with the likelihood of walking and bicycling (Appleyard, 2012). Research on parental decisions found distance to be the top 
factor influencing whether students walked or cycled to school (Zhou et al., 2010). Travel time had a strongly significant and large negative effect on the odds of walking in several formulations of a tour mode choice model (Miller, Roorda, \& Carrasco, 2005). Even for short trips (less than 1.4 miles) trip distance was negatively associated with the odds of walking compared to driving or cycling (Kim and Ulfarsson, 2008). Travel time was a significant negative factor on the joint choices of destination and walk and bicycle modes for personal business, social, and recreational travel purposes (Singleton \& Wang, 2014).

Research also shows that minimizing travel time is of primary importance for route choice while walking and cycling. A study of pedestrian route choice to access rail transit found $99 \%$ of respondents considered choosing the shortest route to be very or somewhat important (Agrawal et al., 2008). Bicycle route choice studies consistently find distance to be a major factor in choice of route (Broach et al., 2012; Hood et al., 2010; Sener et al., 2009). Willingness to deviate from the shortest path is a useful way to evaluate the importance of certain factors with respect to travel time or distance. There may be situations in which longer travel times are positively valued (Mokhtarian \& Salomon, 2001; Ory \& Mokhtarian, 2005; Redmond \& Mokhtarian, 2001; Manaugh \& El-Geneidy, 2013), but these can be accounted for as providing greater enjoyment or pleasure (see the Pleasure need section).

\subsubsection{2.b. Monetary Cost}

An obvious and important cost consideration is money spent during the travel option being considered. Monetary costs for driving may include the costs of fuel, tolls, and parking. Monetary costs of using transit may simply include the costs of the fare (or 
zero, if a pass is already purchased). Bicycling and walking rarely incur direct costs, although a completely rational decision-maker (who is completely fictional) might include the maintenance costs of using a bicycle, the food costs to make up the energy used, or the incremental costs of wear and tear on shoes while walking. Most research suggests that the monetary costs included in travel behavior models should only be those costs directly considered when making the travel decision. For example, a driver might not consider the cost of automobile insurance or wear and tear on her vehicle, but might consider (or discount) the cost of fuel expended during the duration of the trip. Using this logic, walking and bicycling normally have no monetary costs.

\subsubsection{2.c. Physical Effort}

Physical effort is a very real cost, particularly for active modes of travel like walking and bicycling that use human-based energy. Driving and riding transit require significantly lesser quantities of physical exertion. Distance and grade are the two characteristics that primarily affect the energy expended while walking and bicycling; longer distances and steeper grades require greater amounts of energy. Hills may become barriers for people riding bicycles to avoid. While steep streets and staircases may provide short cuts while walking, they also can deter some people, especially those with limited mobility (see the Feasibility section above). Weight is also a factor. Riding a heavier or larger bicycle or carrying packages or groceries can reduce the distances people are willing to bike and walk because of the greater physical effort involved. It is important to note that individuals have different levels of acceptable physical effort; alternatively, individuals may have different perceptions about the cost of a given level of 
physical exertion. Also, in this category, physical effort is simply considered a cost; the exercise benefits of physical activity, including while walking and bicycle, are captured under the physical benefits section of the final need: pleasure.

Besides distance, grade or slope - the steepness of terrain - has been shown to affect active travel. Generally, steeper terrain deters walking and cycling because most travel seeks to reduce time and energy expenditure. Several mode choice analyses found that higher slopes and steeper terrain were associated with decreased odds of walking or cycling (Cervero \& Duncan, 2003; Rodríguez \& Joo, 2005). The estimation of a tour mode choice model found that the total rise along a travel path was negatively associated with the odds of walking (Bomberg et al., 2013). Cyclist route choice research reveals people riding bicycles may be willing to deviate significantly to avoid climbing hills or traversing steep grades. One study found that cyclists were willing to travel more than 0.5 kilometers further to avoid climbing 10 meters of hill (Hood et al., 2011). Another revealed preference survey and cyclist route choice model revealed a willingness to travel about $70 \%$ further to avoid a section of roadway with a $2-4 \%$ grade, almost $300 \%$ further to avoid a $4-6 \%$ grade, and over $1,000 \%$ further to avoid an upslope above $6 \%$ (Broach et al., 2012).

Some studies find that more hilly terrain is actually positively associated with active travel. This finding might arise for leisure or exercise purposes, where steeper terrain provides more of a workout or better views and variety (see the Pleasure need section). A lack of hills was associated with physical inactivity in one study (King et al., 2000), while living in a hilly neighborhood was positively associated with physical activity in another (Brownson et al., 2001). Some bicycle route choice stated preference 
studies found a small preference for slightly hilly over flat terrain, especially for recreation and leisure trips (Sener et al., 2009; Stinson \& Bhat, 2003). While a bicycle intersection volume model found that local average slope had a negative effect on bicycle counts, the effect of terrain was reduced during weekends compared to weekdays (Griswold, Medury, \& Schneider, 2011). Terrain may be a barrier more in how it is perceived. People who perceived hills to not be a barrier were twice as likely to meet their leisure time physical activity requirements, while objectively measurements of hilly terrain had not significant effects (McGinn, Evenson, Herring, \& Huston, 2007).

\subsubsection{2.d. Mental Effort}

In addition to time, money, and physical energy, the costs of travel options may also be weighed with a consideration of the mental effort involved in completing the travel behavior. This is particularly critical when considering new or different travel options than normal. For example, a regular car commuter may consider the mental effort to figure out which bicycle routes are safest or where to park his bicycle too great to switch from his regular travel mode. Conversely, someone who regularly bicycles everywhere may be reluctant to drive downtown simply because she may worry about unfamiliar, overwhelming, or confusing parking regulations. The effects of mental effort may be particularly acute in the case of public transit. A significant amount of mental effort may be required to learn how to use a transit system, remember where the key routes go and where stops are located, and (especially) find out when the next transit vehicle will arrive. In these cases, informational campaigns or technological advances can dramatically reduce the mental cost of certain travel options. GPS-based navigation 
systems in cars and real-time arrival information for transit vehicles can reduce mental effort and make these travel modes more attractive.

Very few studies have attempted to assess the effect of mental effort on active travel (mode) choices, although more frequently for navigation and wayfinding. Some researchers postulate that two important factors in pedestrian route choice are simplicity (the number and complexity of decision points) and the availability of landmarks, which together define the complexity or mental quality of a route (Millonig \& Schechtner, 2007). The importance of simplicity in route choice for pedestrians has been noted (Hill 1982). Space syntax suggests that streets with longer sight lines, fewer turns, and high connectivity should see higher levels of pedestrian activity and greater walking propensities (Baran, Rodríguez, \& Khattak, 2008; Raford \& Ragland, 2004). The importance of landmark saliency on the construction of spatial memory and cognitive maps to aid navigation and wayfinding (usually focused on walking in cities) has been noted by several researchers (Golledge \& Gärling, 2004; Raubel \& Winter, 2002; Tom \& Denis, 2003). More generally, the importance of imagability or legibility of one's urban environment, arguably a cognitive perception involving mental effort, is of importance to travel decision-making (Lynch, 1960; Taylor, 2009).

Less work has investigated the effects of mental effort on cycling. The significant preference for bicycle boulevards or neighborhood greenways in one bicycle route choice analysis - above and beyond aspects such as slope, turns, number of stop signs and traffic signals, and street crossing volumes - might suggest that users place a value on the simplified navigation these facilities provide via directional signs and sharrows (Broach et al., 2012). Mental effort affects other modes as well. Work has shown that spatial 
ability and network knowledge can affect choices of route for auto (Ben-Akiva, Ramming, \& Walker, 1999; Ramming, 2002) and transit (Dziekan, 2008) modes, where maps may be particularly important for the latter (Guo, 2011).

\subsubsection{2.e. Convenience and Reliability}

A final aspect of cost may be more a consideration of future potential costs of changing one's mind or encountering changing conditions. When making travel decisions, people may consider the likelihood that their plans may change and consider what contingency they would have to take. Consider destination choice for shopping travel: if one store does not have the product a person was looking for, it will be more convenient for him to try another store if he has chosen an establishment close to other businesses than one in an isolated location. Consider mode choice: a person may be more likely to ride her bicycle to work if she knows there is a parallel transit line she could use if her bicycle has a mechanical issue. Finally, consider the effect of travel time reliability on time-of-day choice: during rush hour, driving times may be highly variable so a worker may choose to use a transit line on a parallel busway, even though it may take longer on average, simply because travel time on the transit facility is more reliable than on the highway. Other people may also value their freedom and sense of control when traveling, so travel options that sacrifice their ability to travel independently may receive a cost. Convenience, contingency, flexibility, resilience, and reliability are characteristics that may be considered when weighing the potential costs of different travel options.

The positive effect of convenience on travel decisions is often thought to be a factor in favor of driving. Car users have expressed positive feelings of control versus use 
of public transit, feeling self-sufficient and able to start journeying or change route and/or destination with minimal preparation (Gardner \& Abraham, 2007). In another study, while regular car commuters rated the convenience of walking and bicycling as low, people who normally walked and cycled to work rated their own mode's convenience as high; convenience was the attribute with the greatest disparity (Anable \& Gatersleben, 2005). Despite their slower speeds and higher generalized costs for longer trips, active travel modes can be more convenient than driving. Given the right environmental conditions, walking can be a convenient and common way of traveling between multiple stores within shopping districts even for people who drive to the shopping districts (Schneider, 2011).

Most studies looking at the effects of travel time reliability have focused on travel by automobile or public transit (Bhat \& Sardesai, 2006; Brownstone \& Small, 2005; Fosgerau \& Karlstrom, 2010; Hollander, 2006; Lam \& Small, 2001). Travel time variability may be a significant but less important of a factor than travel time itself, and there may be more heterogeneity as to the value of travel time reliability than for travel time (Bhat \& Sardesai, 2006). Other studies find that one minute of travel time reliability is valued higher than one minute of travel time savings (Carrion \& Levinson, 2012). One study found that travel time unreliability had a negative effect on commute mode choice that was more negative for those with inflexible work schedules (Bhat \& Sardesai, 2006). Several reviews of the literature and discussions on travel time reliability have been published (Bates, Polak, Jones, \& Cook, 2001; Carrion \& Levinson, 2012; Li, Hensher, \& Rose, 2010; Noland \& Polak, 2002). 
Little attention has been paid to analyzing the reliability of active modes of travel. Conceptually, walking and bicycling should be more reliable and resilient when it comes to estimates of travel time. At least in the US context, pedestrian and bicycle volumes are low enough that congestion is rare and thus tends to have little effect on travel time reliability, especially where sidewalks are complete and separated bicycle facilities are provided. Similarly, these active travel modes are highly maneuverable when compared to motor vehicles, so travel time by these modes should be relatively resilient in the face of traffic incidents.

\subsubsection{Pleasure}

\subsubsection{Definition}

The last need, pleasure, is somewhat of a catch-all category to describe comfort, enjoyment, and other personal and social benefits of a travel option. Specifically, benefits include the physical benefits of comfort and exercise and the mental and emotional benefits of enjoyment and pleasure (including internalized social benefits). This category of need reflects all of the positive aspects of travel that are to be maximized when making a rational choice. Statistically, all of the variables influencing pleasure should have positive coefficients in the modeled equation. The pleasure need also captures some of the co-benefits or activity value of travel.

This category of needs finds support in several travel behavior theories pertaining to walking and bicycling. The hierarchy of walking needs (Alfonzo, 2005) includes both comfort and pleasure as the two highest (least relevant) needs. Comfort therein is defined 
as a person's convenience, ease, or contentment, while pleasurability described as the interest, aestheticism, and enjoyment afforded by walking. The distinction between comfort and pleasure in the hierarchy of walking needs is not well defined. In this theory's hierarchy of travel needs, convenience and ease are placed within a Cost need, while contentment fits in this Pleasure need. The theory of routine mode choice decisions (Schneider, 2013) also has a category called enjoyment which is closer to what pleasure is considered here. Enjoyment therein is defined as personal (physical, mental, or emotional) benefits, achievement of social status, and subjective norms or benefits to society or the environment.

These considerations of pleasure and enjoyment obtained from travel are not exclusive to walking and bicycling, although different factors may have unique influences. In particular, walking and bicycling (and skating and scooting) provide exercise through the exertion of human energy in quantities greater than that during driving or riding transit. Typical metabolic equivalents (MET) for riding in a car, walking, and cycling are, respectively, 1.3, 3.5, and 7.5 (Ainsworth et al., 2011). People driving, cycling, and walking along a street also perceive the same environment differently, primarily due to differences in speed, attention, and environmental exposure (Frank \& Engelke, 2001). Environmental factors influencing comfort, including temperature, precipitation, shade, noise, and air pollution likely have a greater influence on walking and bicycling. Similarly, the need for attention to the roadway while driving (and bicycling) means less mental effort, when compared to walking, can be spent observing and exploring one's surroundings, enjoying the aesthetic nature of the environment, and socially interacting with companions. 


\subsubsection{Examples and Empirical Support}

Examples of factors that influence pleasure - comfort, exercise, and other physical benefits; enjoyment, pleasure, and other mental and emotional benefits - are described in more detail in the following sections.

\subsubsection{2.a. Physical Benefits: Comfort and Exercise}

Depending on the mode, travel can afford many physical benefits, including but not limited to comfort and exercise. These considerations can be relevant in the travel decision-making process. Some people may consider driving to be more comfortable than riding transit because of ergonomic seats, temperature control, ability to control music or the radio, and/or solitude. Still others may think the opposite, that sitting in a bus or train with the ability to read or work is more comfortable than sitting in traffic trapped by a seatbelt. Walking on the sunny side of the street may be less comfortable than the shaded side in the heat of summer, but a comforting sun may be desired when walking in the cold depths of winter. For the active travel modes, walking and bicycling, another physical benefit may be exercise. In contrast to the cost of physical exertion, some people may decide to walk, run, or ride their bicycle between activities in lieu of participating in separate non-travel exercise-based activities like going to the gym.

There are many environmental factors that may be linked to the comfort of travel, particularly for walking and bicycling. These comfort factors may include traffic volumes and speed, the presence of traffic calming, buffers between walking and bicycling facilities and motor vehicles, plants and animals, street furniture, sunlight, shade, smell, and sound. Riding a bicycle in heavy traffic (while breathing in auto emissions) may be 
less comfortable than riding on quiet tree-lined residential streets. Having a bench upon which to rest, even for a short time, can improve the comfort of a walk through the neighborhood or in a park. Similarly, other sidewalk amenities including ramps, handrailings, drinking fountains, trash cans, awnings, street trees, and street lighting can make walking a more comfortable experience.

Weather may increase the time it takes to travel by active modes, such as the need to put on or remove more layers in winter or change after being in the rain or heat. Weather also affects the comfort of the journey. Many studies have found that cycling volumes vary seasonally, decreasing in cold, wet, and snowy weather and increasing in warmer temperatures (Chen \& Clifton, 2012; Flynn, Dana, Sears, \& Aultman-Hall, 2012; Lindsey, Chen, \& Hankey, 2013; Nordback, Marshall, Janson, \& Stolz, 2013). On the other hand, trees offer protection for pedestrians from the elements, including heavy rain and sun. Research has revealed the importance of trees and landscaping on the choice of route by people walking to access rail transit, although these factors were less important than short routes and traffic safety concerns (Agrawal et al., 2008).

Individual research varies as to the effect of weather on physical activity levels, depending on context and time of year. A recent review found that physical activity varies seasonally and inclement weather can be a barrier to physical activity (Tucker \& Gilliland, 2007). Some studies on physical activity found no effect of weather (Humpel, Owen, \& Leslie, 2002). Others find mixed effects; respondents who reported weather as a barrier to physical activity were more likely to engage in sedentary behaviors, although weather barrier perceptions had no significant influence on physical activity (Salmon et al., 2003). Still others find positive effects moderated by gender; men were much more 
likely to be high exercise walkers and women were much more likely to frequently walk around their neighborhood if they perceived weather to not inhibit their walking (Humpel, Owen, Iverson et al., 2004).

A major study applied the Irvine-Minnesota Inventory (IMI) - a measure of micro-level built environment characteristics hypothesized to be related to walking and cycling - to data on physical activity and walking (Boarnet, Forsyth, Day, \& Oakes, 2011). Several items in the IMI were designed to capture environmental comforts (Boarnet, Day, Alfonzo, Forsyth, \& Oakes, 2006; Day, Boarnet, Alfonzo, \& Forsyth, 2006). Significant results on the effects of these comfort factors were limited. Wellmaintained sidewalks may be more comfortable; sidewalk condition was positively associated with walking for transportation but not for leisure. Besides providing a safety benefit, a buffer between sidewalk and street reduces traffic noise; the presence of a traffic buffer was also positively related to transportation walking but not leisure walking. The noise from high-speed highways may also contribute negatively to active travel; the presence of a freeway over/underpass was negatively associated with leisure walking. Surprisingly, the presence of benches was associated with less physical activity. Other comfort factors were not associated with either physical activity or walking: sun protection, street trees, sidewalk amenities, and lighting. Factors related to transportation infrastructure and traffic levels were more explanatory than aesthetics and comfort (Boarnet et al., 2011).

Recent work in preferences for travel, termed "travel liking", has investigated the possibility that travel can have positive utility (Mokhtarian \& Salomon, 2001; Ory \& Mokhtarian, 2005; Redmond \& Mokhtarian, 2001). There are many instances of 
undirected travel, that is, where travel is the activity and the destination is an ancillary goal at best. Recreational walking, jogging, running, cycling, skating, skateboarding, and other outdoor activities are perfect examples of undirected travel. Although exercise can be a major motivation for travel as an activity, undirected travel may not involve exerting significant physical human energy (e.g., motor vehicle racing), although it is frequently considered a leisure activity (Mokhtarian \& Salomon, 2001). These distinctions over the overlap of activity and travel are related to the distinctions between types of motivation. Intrinsic motivation is performing a behavior for its own value, such as travel liking; whereas extrinsic motivation is behaving to achieve some outside goal, such as reaching an activity destination (Vallerand, 1997).

Some work has attempted to uncover the specific effects and mechanisms of factors affecting travel liking, including the physical benefits of comfort and exercise. In one study, status and independence were the most important attributes positivelyinfluencing overall travel liking, while a hypothesized desire for environmental exposure, scenery, escape, and curiosity were associated with liking active travel modes (Ory \& Mokhtarian, 2005). More generally, attitudes, personalities, and lifestyles have been found to significantly affect travel liking, and socio-demographics also played a role (Ory \& Mokhtarian, 2005). More work is needed to examine the value of exercise for nonrecreational (or directed) walking and cycling transportation, and the effects of comfort on these active travel modes. 


\subsubsection{2.b. Mental and Emotional Benefits: Enjoyment and Pleasure}

In addition to physical benefits like comfort and exercise, travel may also afford mental and emotional benefits, including but not limited to enjoyment and pleasure. While difficult to define and measure, these considerations can also be relevant in the travel decision-making process. Factors that affect mental and emotional benefits are more difficult to define, but may include streetscape and aesthetic characteristics including diversity, complexity, detail, color, architectural style, historic structures or events, statutes and landmarks, building scale, setting, and the presence of views. The benefits of these factors may relate to intangible personal motivations including stressrelief, variety-seeking, aesthetic involvement, personal achievement, belongingness, sacrifice, and helping others. Many people consider walking to be a stress-reliever. Others may choose to walk because it affords them the opportunity to explore and seek new routes to their regular destinations. A particular neighborhood may be popular for walking because of a certain positive aesthetic about the overall design of the streetscape or for particularly aesthetically-pleasing buildings or landmarks. Some people do things to prove they can, such as walking six miles each day to and from work. In some US cities a strong bicycle culture has developed, and bicycling can demonstrate that a person belongs to and wants to be identified with a larger positive cultural group. Benefits to society from one's active travel decisions can also be included in the theory as internalized societal benefits. Many travelers, particularly those who walk or bike, report enjoying and finding pleasure in their travel in part because they see it as making a sacrifice (not driving) in support of a larger external goal, such as improving the natural environment. In some cases, traveling by walking or bicycling can simply be fun. 
Many factors contributing to mental and emotional benefits could be described as the aesthetics of street or urban design. Several scholars and theorists propose more attention be paid to these factors, described as aesthetics by Pikora et al. (2003). Forsyth and Krizek (2011) argued the need for urban design for cycling to move beyond functional considerations of safety and exercise and consider experiential factors. Timms and Tight (2010) suggested a more theoretical consideration of street design aesthetics to make walking and bicycling more pleasurable experiences has potential to encourage more use of active travel modes, but they also noted the impossibility of making "objective" aesthetic assessments. Zacharias (2001) noted other hypothesized perceptive environmental aspects that affect pedestrian travel.

Of contention are the types of urban design considerations of primary importance to the enjoyment of walking and bicycling. Many terms have been ascribed to urban design, including legibility or coherence (ease of understanding, organization, and navigation), complexity (diversity of a scene's elements), mystery (the extent to which a scene invites one to explore deeper), and aesthetics (perception as an end in itself). While perception of legibility is cognitive, aesthetic perception is both cognitive and affective (emotional). Many have suggested the important role of legibility (Lynch, 1960), while some have argued that aesthetics is a more important principle than legibility for determining quality in urban design (Taylor, 2009). A Delphi study of experts suggested that streetscape aesthetics were a more important consideration than views for both walking and cycling, particularly for non-recreation travel (Pikora et al., 2003).

Studies of environmental preferences yield insight into which urban attributes travelers find the most pleasure or enjoyment. Most work in this field involves the rating 
of color images. A study of residential scenes in Pittsburgh found that respondents preferred scenes with ornate buildings, well-kept properties, unambiguous land uses, and less enclosure (Nasar, 1983). A scene's mystery was positively related to preference of both urban and nonurban environments (Herzog \& Smith, 1988). Significant, consistent, and positive predictors of the preference for urban scenes were coherence and complexity (Herzog, 1992). Another study found a preference for viewing houses with popular or traditional architectural styles over modern or atypical styles (Stamps \& Nasar, 1997). Visual diversity or entropy of housing color, scale, and shape were positively associated with respondent pleasantness (Stamps, 2002). When analyzing evaluations of computer generated environmental scenes, impressions of enclosure were influenced more by visual permeability than by environmental permeability (Stamps, 2005).

Aesthetic assessments may vary less than might be expected. A meta-analysis of environmental preference literature found a very high degree of aesthetic consensus among most demographic groups, including by gender and student status, but less consensus among special interest groups and people of different ages (Stamps, 1999).

Research from transportation and public health fields finds some support that enjoyment of environments can affect walking and bicycling. The perception of pleasant streets for walking was only significant as a negative predictor of the fraction of car trips and had no association with the number of fraction of non-motorized trips (Kitamura et al., 1997). Environmental aesthetics were positively related to the odds of walking for exercise in a study of Australian adults (Ball et al., 2001). Perceived measures of environmental aesthetics were more consistently related to walking and physical activity 
(McCormack et al., 2004). Sometimes, the relationship between aesthetic environmental characteristics and walking/cycling had inconsistent findings (McCormack et al., 2004).

Transportation and public health research also begins to point towards environmental factors that influence the attractiveness of active travel facilities and modes. For the most part, significant findings center on the enjoyment of buildings and nature. The presence of interesting houses to look at and seeing other people, especially neighbors, were positively correlated with walking frequency for strolling trips (Handy, 1996). Pedestrian route choice research has revealed the importance of attractive buildings and the presence of other people walking, although these factors were less important than short routes and traffic safety concerns (Agrawal et al., 2008). Residents of high-walkability neighborhoods had more positive perceptions of attractive buildings and natural views in their neighborhood than did residents of low-walkability neighborhoods (Saelens, Sallis, Black, \& Chen, 2003). Perceptions of enjoyable scenery were positively associated with physical activity (Brownson et al., 2001). The presence of deluxe green street storm water infrastructure and the adjacency of parks contributed to the attractiveness of walkable street segments (Adkins et al., 2012).

Travel can also be a beneficial social activity. Having a partner or friends who were physically active was positively associated with physical activity in a study of older Australians (Booth et al., 2000). Adults with no one to accompany them (either a person or a pet) were significantly less likely to walk for exercise; this effect of company was stronger for women (Ball et al., 2001).

Research on travel liking points to affective influences and mental, emotional, and social benefits of travel. Transportation provides opportunities for relaxation, thinking, 
communicating, exploring, and fulfillment of personal needs like curiosity and variety (Mokhtarian \& Salomon, 2001; Ory \& Mokhtarian, 2005). In one survey, some of the biggest reasons for engaging in excess travel were to see beautiful scenery, explore new places, trying a new route to a familiar destination, and just for the fun of it (Mokhtarian \& Salomon, 2001).

Travel liking has been shown for car use and for commuting. Studies have found that car use is attractive beyond its use for transportation; note the US's past "love affair with the automobile". Travel by car is often rated positively compared to other modes (Mokhtarian \& Salomon, 2001). Car use has been rated much higher than public transportation in attractiveness because of perceptions of the independence, freedom, and social status it affords (Steg, 2003). Symbolic and affective motives may influence car use beyond "instrumental" concerns of cost and time, even for commuting purposes (Steg, 2005). Commuting may not always be an activity to be minimized, as it might provide an opportunity to relax, decompress, and pass some time alone in an otherwise busy life. A survey found that a commute time of 15-19 minutes was ideal for the greatest number of respondents (Redmond \& Mokhtarian, 2001).

Positive values of travel have also been found for active modes. The travel liking score for walking, jogging, and bicycling was significantly associated with proenvironment attitudes, family or community-oriented lifestyles, higher educational attainment, and greater frequency of travel for exploration (Ory \& Mokhtarian, 2005). Walking, jogging, and bicycling had more positive of travel liking than other modes (Mokhtarian \& Salomon, 2001). Another study found that bicycling and walking commuters had the highest levels of commute well-being or happiness (Smith, 2013). 


\subsection{Moderation and Indirect Effects: Demographics and Socioeconomics}

\subsubsection{Definition}

Demographics, socioeconomics, and life stage are placed outside of the travel decision-making process because they are beyond the short and even medium term scope of this theory. Some demographic information like age, race, and (to some degree) gender and ethnicity are completely exogenous to all travel decision-making because they cannot be changed. Socioeconomic characteristics - education, occupation, income - rarely change or do so on a long time frame: years. Stages in life are more fungible but still are typically expressed in a longer-term duration than is considered in the travel decisionmaking framework. Life stage changes do lead to dramatic travel changes through participation in different kinds of activities and having different travel needs, so they should be forecast in a more comprehensive and longer-term travel framework (Van Acker et al., 2010).

One important aspect of the theory of travel decision-making is that, following Schneider (2013), life stage and individual demographic and socioeconomic characteristics have no direct influence on travel behavior. This is by design: as travel is directed by a demand for activities and travel alternatives are chosen in relation to their perceived ability to fulfill travel needs, descriptive characteristics of individuals do not otherwise influence behavior. Instead, demographic, socioeconomic, and life stage characteristics have several indirect roles to play in the travel decision-making process. First and foremost, it is hypothesized that they act as moderating influences on the relationships between the hierarchy of travel needs, individual perceptions of the needs, 
and travel decisions. This moderation effect may act primarily through an influence on individual perceptions of travel needs fulfillment, a "mediated moderator" effect described in a later section. Second, socioeconomics and life stage in particular directly inform the feasibility of different travel options. There may be other minor effects not represented in the theoretical framework. Individual demographic/socioeconomic characteristics may affect how past travel decisions and outcomes in the form of habit or exploration feedback to influence current travel decision-making. These influences may also moderate how perceptions of travel needs fulfillment are valued within a decisionrule framework. Finally, although it is outside the scope of this theory, it is important to note that individual characteristics are particularly important in the generation of activities.

First, consider the moderating role of these descriptive individual characteristics. In other words, the impact of the hierarchy of needs on travel behavior varies for people with different demographic, socioeconomic, and life stage characteristics. For example, young parents may place a higher value on safety and security for their family's travel decisions than might college-aged students. In another situation, lower income travelers may prioritize achieving a lower cost over other needs like pleasure and safety/security, whereas higher income travelers may opt to fulfill their pleasure need without worrying about cost. Although outside of the active travel spectrum, higher income and business air travelers may choose to fly first class (pleasure over cost) while lower income air travelers may choose to fly coach in a discount airline (cost over pleasure). Speaking statistically, the effect of the cost need on travel decision-making depends on a person's socioeconomic status. 
This moderation effect may act through an influence of these characteristics on individual perceptions. In other words, this relationship assumes that people with similar demographic, socioeconomic, and life stage characteristics share some common values about the hierarchy of travel needs. People with certain backgrounds and experiences may have more positive attitudes about certain travel modes. Members of other cultural groups may have common negative social norms associated with walking or bicycling. Because of the strong role of social and community beliefs and motivations in defining subjective norms and thus travel attitudes and preferences, demographic and socioeconomic characteristics are thought to influence perceptions of the hierarchy of travel needs. In the absence of measures of travel attitudes and perceptions, individual descriptive characteristics may appear to moderate travel decision-making.

Second, socioeconomics and life stage in particular directly inform the feasibility of different travel options. In other words, socioeconomic and life stage characteristics play a major role in whether a travel option is physically, spatially, and temporally feasible. Socioeconomic status is closely related to vehicle ownership, which affects whether driving or bicycling are available travel mode alternatives. Similarly, some stores may be beyond the acceptable price range for lower-income individuals, so socioeconomic status affects which destinations are feasible. In addition, life stage phase plays a large role in the feasibility of travel flexibility. A family of four, with two working adults and two primary school students, likely has a highly-constrained daily schedule - many coupling constraints (Hägerstrand, 1970) - making typically slower travel modes like walking and bicycling and time-of-day shifting less feasible. 


\subsubsection{Theoretical Support}

These relationships find support in psychological and travel behavior theories. TRA (Fishbein \& Ajzen, 1975) proposes that demographic and socioeconomic factors indirectly affect behavior only through the attitudinal and normative factors that influence behavioral intention. TIB (Triandis, 1977, 1980) shows that situational context or facilitating conditions moderate the influence of intentions and habit on behavior. Other theories - TRB, SCT, IMB, and CADM - suggest that these descriptive individual characteristics, in their role as resources, enabling factors, facilitators, environmental constraints, or situational characteristics, also influence human behavior. Alfonzo's social-ecological framework for walking (Alfonzo, 2005) shows individual (demographic, biological, and psychological) and social (sociological and cultural) factors moderating the relationship between the hierarchy of needs and walking behavior. Schneider (2013) proposes that socioeconomic factors only indirectly influence routine mode choice decisions through their influence on how individuals differ in their interpretation of modal tradeoffs, similar to the hierarchy of travel needs.

Conventional discrete choice travel demand models based on random utility maximization make assumptions about the relationship between demographic and socioeconomic characteristics and travel decisions. They suggest that the systematic utility function $V_{i n}$ for alternative $i$ and decision-maker $n$ can be specified to be a function of alternative attributes $\mathbf{z}_{\mathbf{i n}}$ and decision-maker characteristics $\mathbf{S}_{\mathbf{n}}$. In basic mathematical form, the linear-in-the-parameters utility function allows sociodemographic variables to enter directly, as transformations of themselves, or as interactions with attributes of alternatives: $V_{i n}=V\left(\mathbf{z}_{\mathbf{i n}}\right)+V\left(\mathbf{S}_{\mathbf{n}}\right)+V\left(\mathbf{z}_{\mathbf{i n}}, \mathbf{S}_{\mathbf{n}}\right)$. Common 
decision-maker characteristics found to influence mode choice, for example, include the age, gender, and race of the traveler, and the income, number of autos, number of workers, number of adults, and number of children in the household (Ben-Akiva \& Lerman, 1985; Koppelman \& Bhat, 2006; Train, 2009). Demographic and socioeconomic characteristics like vehicle ownership, household income, and household size are also key factors used to forecast the demand for walking and bicycling in nearly all US regional travel demand models (Singleton \& Clifton, 2013).

\subsubsection{Empirical Evidence}

Plenty of empirical evidence supports the theory's hypothesis that demographic, socioeconomic, and life cycle characteristics directly influence the feasibility of travel alternatives. The primary example of this is vehicle ownership. In travel demand models, household auto ownership is usually a defining variable in whether or not driving is an available travel mode option. Auto ownership has been closely related to car use, and both household income and life cycle stage influence auto ownership (Giuliano \& Dargay, 2006). Similarly, bicycle ownership and bicycle use are closely linked (Handy, Xing, \& Buehler, 2010). Having a mobility limitation as a result of a disability or health issue has been linked to lower levels of walking and physical activity (Berrigan \& Troiano, 2002). These socio-demographic variables likely affect certain travel decisions, such as travel frequency, greater than they do other travel decisions like destination or mode choice (Hanson \& Hanson, 1981).

Research also supports the moderation hypothesis, although it is less common for travel demand models to explicitly include interactions between socio-demographic and 
travel alternative characteristics in the utility function (Koppelman \& Bhat, 2006). One exception is the use of income as a moderating variable. More often, socio-demographic factors are modeled as control variables without postulating their role in a theoretical travel behavior framework. Less-frequently - with some exceptions (e.g., Reilly \& Landis, 2003) - is it acknowledged that the inclusion of direct socio-demographic variables in travel demand models is actually intended to account for their influence on taste variation in place of actual measurements of travel attitudes and preferences (Koppelman \& Bhat, 2006).

The theory's hypothesis that demographic, socioeconomic, and life stage characteristics moderate the relationship between environmental and travel attributes and travel behavior has some empirical support, primarily from demographic effects like age. Studies focusing on the walking behavior of children have yielded environmental factors whose influence varies with age (Saelens \& Handy, 2008). Pedestrian infrastructure and traffic safety factors may play a greater role for children in decision of walking to school, while proximity and population density factor larger into adults' decisions (Saelens \& Handy, 2008; Timperio, Crawford, Telford, \& Salmon, 2004). Similarly, studies focused on older adults and the elderly have also revealed significant travel behavior differences for this population, including greater auto dependency and greater sensitivity to accessible walking environments (Cao, Mokhtarian, \& Handy, 2010; Kim \& Ulfarsson, 2004; Paez, Scott, Potoglou, Kanaroglou, \& Newbold, 2007). One study on walking found significantly-different effects of the built environment on walking behavior of people with different characteristics, including differences by gender, race, education level, auto ownership, number of children, employment status, and health indicators 
(Forsyth, Oakes, Lee, \& Schmitz, 2009). Many other socio-demographic characteristics likely moderate these relationships, although it is more difficult to uncover them than direct effects on travel behavior. It often takes a much larger sample to find statisticallysignificant interactions terms between travel attributes and decision-maker characteristics (Cohen, Cohen, West, \& Aiken, 2003; Snijders \& Bosker, 2012).

There also exists some evidence that demographic characteristics moderate perceptions of the hierarchy of travel needs or the attitudes, norms, and self-efficacy that generate those preferences. Gender differences have been studied to some extent, and there is evidence that women have stronger ecological norms and preferences for public transportation (Matthies, Kuhn, Klöckner, 2002) and different perceptions of the neighborhood environment than men (Humpel, Owen, Leslie, et al., 2004). One study even found gender differences in perceptions of weight-related barriers to physical activity (Ball, Crawford, \& Owen, 2000). With respect to walking, women may be more sensitive to perceptions of the pedestrian environment, especially to concerns of traffic safety and security from crime (Clifton \& Dill, 2005; Kim, Ulfarsson, \& Hennessy, 2007). Socio-economic status has been found to influence children's perceptions of neighborhood safety (Timperio et al., 2004). There may be a number of other demographic characteristics - such as race, age, and education - that also moderate individuals' perceptions of the hierarchy of travel needs.

Demographics and socioeconomic characteristics also affect activity demand, although this relationship is outside of the travel decision-making process and thus beyond the scope of this theory. Research has shown that household size and type, individual age and gender, worker and student status, and intra-household interactions all 
significantly affect the activities in which a household engages (Ben-Akiva \& Bowman, 1998b; Chapin, 1974; Lu \& Pas, 1999; Pas, 1985).

\subsection{Mediation: The Perception Lens}

\subsubsection{Definition}

The so-called "perception lens" is an important construct within the travel decision-making process. It is an intermediary between the objectively defined components of the hierarchy of travel needs and the evaluative process that leads to travel decisions and outcomes. Social norms (which include subjective norms) and individual or personal values, beliefs, attitudes, preferences, and perceptions explain differences in how people weigh the different travel needs with respect to the travel option under consideration. Of course, perceptions of travel needs fulfillment may also differ according to the specific situation, including by trip purpose or time of day. In other words, the perception lens mediates the relationship between the hierarchy of travel needs and observed/chosen travel behavior.

More specifically, the perception lens acts to adjust how individuals view the fulfillment of each of the travel needs and their relative value. It is perceptions of the fulfillment of travel needs that are weighed when individuals evaluate travel options, not the measured variables within the hierarchy of needs themselves. Conceptually, different people may have varying assessments of whether a travel option affords them a reasonable level of safety and security, or someone may value cost over pleasure in certain situations. Operationally, different people think a travel option does or does not 
provide safety and security, or someone only considers cost and does not consider pleasure. Statistically, while there is a hypothesized significant relationship between the variables in the hierarchy of travel needs and observed travel behavior, the theory also suggests a strong and significant relationship between individual perceptions of affordances and observed travel behavior. In the theory of travel decision-making, the direct effect of individual and environmental factors (arranged according to the hierarchy) is fully mediated by perceptions of those factors and the fulfillment of those needs.

According to the theory, after the characteristics and attributes of travel alternatives are defined and measured, the decision-maker considers the value of each alternative as expressed by perceptions of the hierarchy of travel needs. In the absence of measures of individual perceptions of needs fulfillment, demographic and socioeconomic characteristics may appear to moderate the influence of the hierarchy of travel needs on travel behavior. People of similar age, gender, or income may share similar travel values and make similar travel decisions; older people may be more likely to walk than to cycle. However, there is still considerable individual variation in such travel decisions; the theory justifies these variations as differences in perceptions. Travel perceptions are said to mediate the relationship between the hierarchy of travel needs and travel behavior. Indeed, these perceptions (depending on how they are measured) may partially or fully explain the hypothesized moderation effect. In psychological statistical literature, this complex set of relationships is called "mediated moderation" (Morgan-Lopez \& MacKinnon, 2006; Muller, Judd, \& Yzerbyt, 2005; Preacher, Rucker, \& Hayes, 2007). 
Examples assist in the explanation of the mediation process. The simplest example is explaining the value of time. Travel time is an objective measure of the cost or convenience of a travel option. For various reasons (e.g., income, personal values), different people have different perceptions of the importance of minimizing travel time. For some, a five minute savings in commute time is worth a great deal; for others, the same travel time savings is nearly worthless. This worth can be expressed as the value of time. The perception concept might also explain why some people arrive consistently late to appointments: because their perception of what is feasible may not match reality, their travel behavior reflects this infeasibility by not arriving on time.

Other more complex examples may further help to explain how this process works. Consider two workers deciding between driving and riding a bicycle to work. Both options are objectively feasible from time/schedule and accessibility/mobility perspectives: there is time to make either journey and both a car and a bicycle are available. One person decides to ride her bicycle because she perceives that the bicycle lanes will provide her enough safety from traffic and wants to save the monetary costs of driving. The other person decides to drive his car because he perceives that the bicycle lanes are not safe enough and he has a negative subjective norm (or a perceived negative social norm) for bicycling because he thinks his coworkers or boss expects him to arrive in business dress, not having cycled. While built and natural environment factors are the same, individual perceptions affect the travel decision.

Now consider two high-school students faced with the options of walking versus talking the bus to school. Again, both options are objectively feasible from time/schedule and accessibility/mobility perspectives: there is enough time before school starts for 
either option and there are no major-street barriers to walking. One person may decide to walk, even though the cost in time and physical expenditure is greater, because she feels safe in the neighborhood and enjoys the outdoors. The other person may decide to take the bus not because it would save time or be safer but because he has low confidence or self-efficacy about walking; he thinks walking would take too long and be infeasible because he has never tried it before. In both cases, personal factors mediate the effect of variables in the hierarchy of travel needs on travel behavior decisions and outcomes.

The perception lens construct also accommodates limited decision-maker knowledge about the existence of travel options or misinformation about characteristics of those options or the travel situation. For example, a person may not know that taking the bus is an option because she does not have the information that suggests that riding transit is available; to her, riding transit is infeasible due to a lack of knowledge. Alternatively, a person who only drives to a routine destination may underestimate the time for him to drive there but overestimate the time it would take to walk there, thus biasing his evaluation of the two modal options; to him, walking seems to costs more than it actually would. Providing accurate information about the availability of transit service and the estimated walking time could change these misperceptions.

There may be a number of ways in which perceptions of travel needs fulfillment may be formed. Like TBP (Ajzen, 1991), attitudes, subjective norms, and perceived control - all shaped by personal beliefs and evaluations - may influence how those hierarchically organized factors within each travel need are perceived as fulfilling or affording such needs. In addition, as this theory suggests, new information obtained via exploration (new travel choices) or some sort of intervention (e.g., educational and 
informational campaigns, peer group input) can change these perceptions. Such perceptions have been developed in an individual over a lifetime, with influence from demographic and socioeconomic characteristics as well as one's social environment (Bandura, 1986) and general travel experience.

It may be useful to define some social-psychological terms related to the perception lens as used in this thesis. A norm is a socially-accepted rule or standard of behavior. A value is a personal principle or standard of behavior. A belief is a strong personal conviction held as true. An attitude is a settled feeling towards something. A preference is a liking for something. A perception is an interpretation or way of regarding something. Together, the theory suggests that norms, values, beliefs, attitudes, and preferences somehow shape individual perceptions of the fulfillment of travel needs.

For the purposes of the theory of travel decision-making, these determinants of perceptions of travel needs (e.g., norms, values, beliefs, attitudes, preferences) are considered exogenous and constant (at least during one decision context); their formation and action are beyond the scope of this theory. It is enough to say that an individual uses his or her own perceptions to evaluate the fulfillment of travel needs. This is not meant to deemphasize the importance of the process of perception-formation; indeed, this process is of critical importance to the evaluation of certain targeted intervention strategies. However, defining such a process is beyond the scope of this travel behavior theory and may be better left to other researchers with more experience in this area. For applications to travel modeling and forecasting, it may be acceptable to represent the perception lens as somewhat of a "black box" (McFadden, 2001). 


\subsubsection{Theoretical Support}

Theories to explain travel behavior and models to forecast travel demand rarely build in components to suggest how different people consider objectively-measured attributes of travel options. Often frameworks only include the most important variables or assume all travelers or groups of people have similar assessments. Occasionally separate models are developed for different narrowly-defined market segments, or certain variables are segmented by income in a pooled model (Ben-Akiva \& Lerman, 1985). One exception is the social-ecological framework for walking (Alfonzo, 2005). Alfonzo suggests that perceptions of the environment mediate the relationship between the hierarchy of needs and walking; individual perceptions, habits, and motivations explain variations in assessments of the fulfillment of walking needs.

The mediation hypothesis of perception finds a stronger basis in psychological theories of behavior. TRA (Fishbein \& Ajzen, 1975) suggests that attitudes and subjective norms affect behavioral intentions and personal beliefs, assessments of outcomes, and motivations determine attitudes and norms. TRA also predicts that environmental factors (the hierarchy of travel needs) operate indirectly on behavior through these other intentional constructs. TPB (Ajzen, 1991) adds perceived behavioral control to influences of behavioral intention, also affected by personal perceptions and beliefs. TIB (Triandis, 1977, 1980) adds affective or emotional factors. SCT (Bandura, 1986) includes outcome expectations (attitudes and subjective norms), self-evaluative outcome expectations, and self-efficacy to explain behavior. In the motivation stage of NDM (Schwartz \& Howard, 1981), internal values, personal norms, externally-imposed values, and social norms help to determine whether a person is motivated to act. All of 
these psychology theories support the hypothesis that there are intangible factors which determine how people view situational considerations, and that these personal factors are the direct determinants of (travel) behavior, with objective situational (environmental) factors acting indirectly through them.

\subsubsection{Empirical Evidence}

There exists much evidence from travel behavior research that personal perceptive aspects, including self-efficacy, norms, values, beliefs, attitudes, and preferences partially or fully mediate the relationship between individual and environmental characteristics in the hierarchy of travel needs and observed travel behaviors and transportation outcomes. Much recent work has focused on addressing the impact of attitudes on travel behavior. Studies have shown direct relationships between modal attitudes and mode choice or frequency of travel by different modes (Bamberg, Ajzen, \& Schmidt, 2003; Bamberg, Rölle, \& Weber, 2003; Galdames et al., 2011; Reibstein, Lovelock, \& Dobson, 1980; Verplanken, Aarts, van Knippenberg, \& van Knippenberg, 1994). These attitudebehavior relationships can be strong or weak, depending on the travel decision situation, attitude measures, and inclusion of other control variables. Other studies, looking at the impact of residential self-selection, have shown that attitudes towards certain travel modes may have stronger effects on travel demand by those modes than do environmental factors (Bagley \& Mokhtarian, 2002; Handy, Cao, \& Mokhtarian, 2005; Kitamura, Mokhtarian, \& Laidet, 1997). Concerns over the sometimes low correlation between attitudes and travel behavior and the low predictive validity of attitudinal measures have led to attempts to include non-preferential concepts into the process - 
such as habit discussed in a later section - and propose better attitude measures (Bohte, Maat, \& van Wee, 2009; Gärling et al., 1998).

Less work has gone into investigating the role of normative influences and perceived control on travel behavior. Some studies have shown direct relationships between personal, social, and ecological norms and travel mode choice (Bamberg, Ajzen, \& Schmidt, 2003; Bamberg, Hunecke, \& Blöbaum, 2007; Bamberg, Rölle, \& Weber, 2003; Klöckner \& Matthies, 2004; Matthies et al., 2002; Wall et al., 2007) or physical activity (De Bourdeaudhuij, Teixeira, Cardon, \& Deforche, 2005). Self-efficacy, or perceived behavioral control, has been shown to directly influence travel decisions like mode choice (Bamberg, Ajzen, \& Schmidt, 2003; Bamberg, Rölle, \& Weber, 2003) or partially mediate the relationship between the environment and physical activity (De Bourdeaudhuij et al., 2005).

Finally, some studies have found that perceptions of the built environment better explain pedestrian behavior than at least some objective measures of the environment (Ball, Bauman, Leslie, \& Owen, 2001; Cao, Handy, \& Mokhtarian, 2006; Humpel, Owen, Iverson, Leslie, \& Bauman, 2004; Humpel, Owen, Leslie, et al., 2004; Livi Smith, 2009; Troped et al., 2001), further supporting the construct of a mediating perception lens in the theory of travel decision-making.

\subsection{Decision Rule}

Travel requires a set of decisions to be made about aspects such as destination, mode, time-of-day, route, frequency, etc. (see the later Decision Contexts and Outcomes section). In the theory of travel decision-making, as well as in other conceptualizations 
(Ben-Akiva \& Lerman, 1985), this decision is framed as an outcome of a sequential decision-making process involving a series of steps. A common process for theories of (travel) choice is to: 1) define the choice problem; 2) generate alternatives; 3) evaluate attributes of alternatives; 4) make a choice; and 5) implement that choice (Ben-Akiva \& Lerman, 1985). The theory of travel decision-making postulates that: 1) an activity defines the decision problem (travel, generally); 2) alternatives may be filtered through the hierarchy of travel needs; and 3) attributes of the alternatives (described by components of the hierarchy's need categories) are weighed based on individual perceptions.

The next step is 4) making a decision, which requires, for an implementable conceptual framework, a decision rule. Such a rule translates a cognitive (or partiallyunconscious) decision process into a code-able heuristic that represents and replicates the hypothesized choice behavior. A considerable number of psychological theories have been developed to explain how motivations or intentions influence human behavior (Ajzen, 1991; Fishbein, 1980; Fishbein \& Ajzen, 1975; Maslow, 1943, 1954; Montaño \& Kasprzyk, 2008; Triandis, 1977, 1980). Arguably, intention is closely linked or even equivalent to decision; although, most (revealed-preference) work in transportation equates decision (choice) with observed (chosen) behavior, while these theories allow other factors (e.g., habit) to affect the relationship of intention and behavior. In fact, a strain of psychology theories regarding behavioral change (Prochaska \& Velicer, 1997; Schwartz \& Howard, 1981) try to fill in this decision-making gap by defining stages of change between motivation and behavior or action. None of these theories define an explicit rule by which an action is taken. 
For a theory of travel decision-making to be useful in informing travel demand modeling and forecasting (a key goal of this thesis), decision rules that can be translated into mathematical equations are necessary. This theory does not postulate a specific single decision rule by which all travelers make decisions; such questions are left to future research. Decision-makers may use any number of decision rules, and one traveler may use multiple decision rules. Multiple decision rules may be used in sequence during one decision process. A decision rule may differ based on the travel decision context (mode vs. route), purpose (commute vs. leisure), and other situational factor. The theory also postulates that habit influences which decision rule is chosen or how strongly an intentional decision rule factors into a travel decision.

Ben-Akiva and Lerman (1985) placed a number of decision rules into four categories: dominance, satisfaction, lexicographic, and utility rules. A dominance rule can be used to eliminate alternatives that are no better than another on multiple criteria. For example, if cycling is just as feasible, just as accessible, just as fast, cheaper, just as safe, and more pleasurable than taking public transit, then the transit mode alternative would be eliminated. A satisfaction rule sets levels of attainment that an alternative needs to meet (or not exceed) to be considered. For example, a time satisfaction rule might eliminate walking as a mode alternative if it took longer than was feasible within a person's schedule. A lexicographic rule could rank all alternatives and attributes and select the best alternative (or eliminate the worst alternative) for the most important attribute. For example, if safety and security were most important for cycling, one might select the route affording the greatest protection from motor vehicles, even if it was not the shortest. Finally, a utility rule assumes that all attributes of an alternative can be 
converted to a scalar value that is to be maximized. For example, if walking to the store took twice as long as cycling but was twice as enjoyable, and pleasure was weighted higher than time for this trip, then walking would have the greatest utility and would be (more likely to be) chosen.

A key distinction between types of decision rules is the difference between compensatory and non-compensatory ones. Compensatory decision rules allow for comparisons or tradeoffs to be made between evaluations of different attributes. An overabundance of one attribute can compensate for a deficiency in another attribute. In the example above, the pleasure of walking outweighed the extra time it took over cycling. Non-compensatory decision rules do not allow such tradeoffs between attributes. Furthermore, non-compensatory rules can be either conjunctive or disjunctive. Both involve establishing criterion levels on attributes, but conjunctive rules require all considered alternatives to meet all attribute criteria, whereas disjunctive rules require only one (usually more stringent) attribute criterion to be met (Svenson, 1979). Dominance, satisfaction, and lexicographic rules are non-compensatory, while utility rules are compensatory (Ben-Akiva \& Lerman, 1985). A number of other decision rules and categories have been proposed and discussed in a variety of academic fields (Gilbride \& Allenby, 2004; Svenson, 1979; Tversky, 1972a, 1972b).

The predominant decision rule used in analyzing transportation choice behavior (and choice behavior in many other fields) is utility maximization. Using work from multiple fields (Luce, 1959; Marschak, 1960; Thurstone, 1927), McFadden derived equations describing a discrete-choice model of random utility maximization, or RUM (McFadden, 1973, 2001). Some of the first applications of RUM were to travel demand 
modeling (Domencich \& McFadden, 1975; McFadden, 1973, 1974). Since then, RUM has been the basic decision-making framework within operational and exploratory travel demand modeling and forecasting (Horowitz, Koppelman, \& Lerman, 1986; Koppelman \& Bhat, 2006; Pas, 1985). RUM has been so widely used because of its strong background in theory, its successful application predicting many types of human behavior, and its ability to be formulated to afford relative ease of mathematical and statistical analysis and model estimation (Ben-Akiva \& Lerman, 1985; Koppelman \& Bhat, 2006).

The formulations derived from RUM do not necessarily assume a utility maximizing decision rule, and thus they can be used to approximate a number of other decision rules (Train, 2009; Tversky, 1972a, 1972b). While this may be acceptable for the purposes of forecasting, it is less useful for an understanding and development of travel behavior theories. As Gärling, Kwan, and Golledge note: "Thus models tend to be confined to specifying what factors affect the final choice, whereas the process resulting in this choice is largely left unspecified" (Gärling et al., 1994, p.356). Some activitybased travel modeling systems have tried to accommodate non-utility-based decision rules, including STARCHILD (Recker, McNally, \& Root, 1986a, 1986b), but operational activity-based models rely on utility maximization for most decisions. Limited research has suggested that non-compensatory decision rules may be at work in transportation decisions and travel behavior (Foerster, 1979; Recker \& Golob, 1979; Swait, 2001; Swait \& Ben-Akiva, 1987a, 1987b; Young, 1986).

Some work on non-compensatory travel decision rules has been conducted and applied to route choice (Bovy, 2009), where the formation of a choice set of considered 
alternatives is a critical issue due to the large number of possible alternatives. Many repeated shortest-path methods exist (Fiorenzo-Catalano, 2007), including labeling. The labeled routes method (Ben-Akiva, Bergman, Daly, \& Ramaswamy, 1984) considers only those routes that optimize a particular single criterion (e.g., quickest, straightest, safest). Constrained enumeration approaches (Hoogendoorn-Lanser, Bovy, \& van Nes, 2007; Prato \& Bekhor, 2006) consider all routes that meet set constraints (e.g., maximum number of turns). Recently, probabilistic or stochastic route choice set generation methods have appeared (Bovy \& Fiorenzo-Catalano, 2007; Frejinger, Bierlaire, \& BenAkiva, 2009). Choice set formation rules and algorithms have been applied specifically to route choice for walking (Hoogendoorn \& Bovy, 2004; van der Waerden, Borgers, \& Timmermans, 2004; Verlander \& Heydecker, 1997) and cycling (Broach, Dill, \& Gliebe, 2012; Broach, Gliebe, \& Dill, 2010; Broach, Gliebe, \& Dill, 2011; Hood, Sall, \& Charlton, 2011).

\subsection{Decision Contexts and Outcomes}

As previously mentioned, the final stage of the theory of travel decision-making involves: 5) implementing the travel decision. The decision rule implements an intention into an action (and behavior) or inaction. This decision (often a choice), in one or more travel decision contexts or dimensions, results in an outcome. For example, if a traveler has decided to walk to a local grocery store, then they have chosen walking as their mode and the store as their destination. This journey then has travel outcomes, including the time spent, distance traveled, and energy expended during the walking trip. 
The theory of travel decision-making intentionally does not specify to which travel decision context(s) it applies. The typical short-term decisions analyzed by regional travel demand forecasting models are choices of frequency, destination, mode, and route; these decision contexts correspond to four-step trip-based model stages of trip generation, trip distribution or destination choice, mode choice, and trip assignment or route choice (Ortúzar \& Willumsen, 2011). Activity-based models (and some trip-based models) also represent related travel decisions such as time-of-day, day activity pattern, primary activity purpose and location, tour mode choice, intermediate stops, and joint travel by household members (Bowman \& Ben-Akiva, 2001; Bradley \& Vovsha, 2005). Integrated land use and transportation models often look for longer-term decisions such as vehicle ownership, residential location, and firm location that are outside of the scope of this theory of (short-term) travel decision-making. Perhaps some simplified form of this travel decision-making framework may be considered during these larger decisions (Van Acker et al., 2010).

The flexibility provided by the multiple dimensions of travel decision contexts explained by this theory allows for the conceptualization and analysis of joint or simultaneous multi-dimensional choices. Most travel model systems and analyses assume a sequential decision-making process (or at least a sequential representation thereof): destination, mode, and route choice. Later choices are conditional upon prior (and concurrent) choices: e.g., mode upon destination, route upon destination and mode. Some feedback mechanisms can be included to allow downstream decisions to influence upstream decisions. Alternatively, travel decisions can be made simultaneously across several dimensions: e.g., destination and mode, mode and time-of-day. A number of joint 
travel choices have been analyzed and modeled (Adler \& Ben-Akiva, 1976; Ben-Akiva \& Bowman, 1998b; Ben-Akiva \& Lerman, 1985; Bhat, 1997), particularly investigating the issue of residential self-selection and endogeneity of household location (Abraham \& Hunt, 1997; Bhat \& Guo, 2007; Lerman, 1976; Mokhtarian \& Cao, 2008). Some studies have specifically looked at joint or simultaneous walking and bicycling choices (Pinjari, Pendyala, Bhat, \& Waddell, 2011; Singleton \& Wang, 2014).

\subsection{Feedback: Habit and Exploration}

\subsubsection{Definition}

A challenging aspect to represent is the feedback effect of past travel decision and outcomes on current travel decision-making. In the theory of travel decision-making, the feedback effect is outside of the travel decision-making process because this process is defined as a short term daily, tour-based, or trip-by-trip decision-making scheme. The influence of past travel behavior is hypothesized to impact travel decisions on a medium term temporal scale: days, weeks, or months. The impact of these previous travel decisions on current travel decision-making can be distinguished into two categories of effect. Habit, or repeated past travel decisions, provides inertia for a particular travel option. Exploration, being faced with a new travel decision or trying a different travel alternative than normal, provides new information about a travel option.

In the theory of travel decision-making, habit is hypothesized to have an inertial effect on the otherwise intentional travel decision-making process, represented as an input to the decision rule. Simply making the same travel decision repeatedly could 
decrease how strongly the hierarchy is consciously considered. For example, the more a person commutes by bicycle to work, the less he consciously considers different travel alternatives. In a more extreme case, habit could completely bypass any evaluative consideration. For example, a person who regularly uses a bicycle to get to work or school may unconsciously grab his bike when going to the local convenience store, even if it would make more sense to walk there. Another possible effect is not included in the theory: habit could change the value of different hierarchical needs. Repeating the same travel behavior could lead to small optimizations and higher value being placed on higher-order travel needs. For example, a person who commutes by bicycle may end up placing a higher value on pleasure or gain more negative perceptions of other travel modes like driving.

The hypothesized effect of exploration is to provide better information and knowledge about a particular travel option, in order to make a more informed travel decision. New information could bring perceptions about objective factors like safety or time more in line with reality. For example, a person walking home from work for the first time may find that it takes much less time or energy than he expected, changing his perceptions of the cost of walking. It could raise awareness of a travel option previously not considered feasible. For example, a person may not realize that a new off-street path opened that she could use to ride her bicycle to school, changing her perceptions about the feasibility of bicycling. Beliefs, attitudes, and travel preferences could also change after being exposed to new information through exploration. For example, the first-time walker mentioned above may also find that he prefers to get fresh air by walking home, changing his preferences of the pleasure of walking. Another reason for choosing a new 
or different travel option is to double-check that a repeated travel behavior is best, the reinforcement explanation. For example, a regular bicycle commuter may choose to try a different route to make sure that she does not prefer it over her normal route, reinforcing her preferences. Repeated reinforcement of perceptions over time can lead to habit formation. Finally, exploration could simply satisfy a need for change, the varietyseeking explanation. For example, a person may decide to take a different route home from work to get a refreshing change of scenery. In the theory of travel decision-making, the impact of exploration feeds new information back into the perception lens to reflect changes in how the hierarchy of travel needs is perceived and valued.

\subsubsection{Theoretical Support}

Several psychology theories have attempted to include habit as an influence on behavior in different unsatisfactory ways and with limited success. Most, such as IBM and CADM, simply suggest that habit directly influences behavior in addition to intention. In TIB (Triandis, 1977, 1980), habit mediates the relationship of intention on behavior. In TTM, behavioral reinforcement (similar to habit) helps to maintain a healthy behavior. TRB (Ronis et al., 1989) focuses its gaze directly on explaining repeated behavior, proposing that habit is the primary unreasoned influence on behavior. TRB suggests that although behavioral intention may be the initiator of a particular behavior, habit determines the persistence of that behavior when faced with repeated similar scenarios. Although travelers may consciously consider their perceptions of the hierarchy of travel needs the first time they decide how to travel to routine activities like work and school, after a few times they may settle into a routine wherein habit directs their travel 
decision-making rule, not the hierarchy or perception thereof. This is similar to the role of habit as a feedback mechanism in the theory of routine travel decisions (Schneider, 2013).

\subsubsection{Empirical Evidence}

Much recent evidence - especially examining travel mode choice - supports the hypothesis that habit does not bypass the decision-making process but simply moderates the intention-behavior relationship. In other words, habit reduces the influence of the conscious travel decision-making process that includes the hierarchy of travel needs acting through the perception lens. Several studies showed that people with strong habits use less information or a less-deliberate decision-process when considering travel mode (Aarts \& Dijksterhuis, 2000; Aarts et al., 1997; Aarts, Verplanken, \& van Knippenberg, 1998; Klöckner et al., 2003). Others have found that habit is a stronger influence on travel mode choice than attitudes, level-of-service variables, and socioeconomic characteristics like auto ownership (Domarchi et al., 2008; Thøgersen, 2006). A handful of studies explicitly tested for and found a significant moderation or interaction effect of habitual bicycle or car use on the relationship between intention and travel mode choice (Gardner, 2009; Klöckner \& Blöbaum, 2010; Klöckner \& Friedrichsmeier, 2011; Klöckner \& Matthies, 2004; Verplanken et al., 1994). Habit and inertial have also been shown to play a role in the choice of route while traveling (Borgers, Viti, \& Hoogendoorn, 2005).

In a few cases, habitual travel behavior has been found to change individual's preferences for the fulfillment of travel needs. In the face of a new carpool lane, solo- 
driving commuters increased their value of flexibility and decreased their consideration of travel cost (Van Vugt, Van Lange, Meertens, \& Joireman, 1996). When subjects in one study were informed that they drove more than they planned, the respondents reduced their pro-environmental attitudes (Tertoolen, van Kreveld, \& Verstraten, 1998). Another study found evidence supporting that mode choice behavior affects modal attitudes (Reibstein et al., 1980). Consistent with the theory of cognitive dissonance (Festinger, 1957), habit may change attitudes and preferences to be more in accordance with available travel options.

Empirical studies have also shown that interventions - letting people try a new travel mode, such as walking or bicycling, or simply providing new information - can be successful in affecting behavioral change and influencing future travel decisions. Several intervention studies which provided a free transit pass to university students documented that the effect of habitual car use on mode choice was weakened (Bamberg, Ajzen, \& Schmidt, 2003; Fujii \& Kitamura, 2003). Similar effects away from habitual car use towards walking and bicycling might be expected as a result of Safe Routes to School initiatives, such as walking/bicycling school buses (Kingham \& Ussher, 2007). Even the injection of new information into the travel decision-making process, whether or not it results from a new travel action, may be enough to affect behavioral change. Studies that forced respondents to deliberately make and justify travel decisions found that the intervention weakened the association between habit and car use (Eriksson, Garvill, \& Nordlund, 2008) or decreased car use among those with prior strong car habits (Garvill, Marell, \& Nordlund, 2003). Feedback and statistics from passive smartphone travel data collection were shown to affect changes in both intention and behavior with respect to 
mode choice (Jariyasunant et al., 2013). New information has even been shown to fully mediate the effect of habit on mode choice when paired with a new decision context such as a residential relocation (Bamberg, 2006; Bamberg, Rölle, \& Weber, 2003; Verplanken, Walker, Davis, \& Jurasek, 2008). These results suggest that targeted informational campaigns can be successful in changing travel behavior and travel decision-making.

\subsection{Conclusion}

This chapter presented a conceptual framework for a unifying theory of travel decision-making specifically designed to address influences on walking and bicycling. It applies only to passenger transportation, not freight transportation, and only for travel within a city, urban area, or region, not for inter-city travel. The theory laid out a possible process by which individuals choose how to travel, as well as categories of individual and environmental factors involved. Seven major components and relationships were described in detail, along with supporting theoretical and empirical evidence:

1. Activity: Demand for travel is derived from a demand for activities.

2. Hierarchy of Travel Needs: Categories of factors influence travel decisions.

3. Moderation: Demographic, socioeconomic, and life stage factors moderate.

4. Mediation: Individual perceptions of travel needs fulfillment mediate.

5. Decision Rule: People may use different rules to make decisions.

6. Decision Contexts and Outcomes: This theory applies to many, even multidimensional, travel decisions.

7. Feedback: Past travel outcomes influence the current decision-making process. 
The theory of travel decision-making has several limitations. A primary limitation is due to the theory's temporal operation and its interaction with other travel-related decisions. Because the theory concerns itself with short-term day-to-day travel decisions, it considers longer-term decisions such as vehicle ownership and residential location to be exogenous factors in the decision process. Any interactions that may occur between an individual's short-term travel decisions, medium-term location decisions, and long-term lifestyle decisions (Van Acker et al., 2010) are outside of the scope, and studies testing this theory would miss these potential relationships. Placing this theory within an interactive multi-level travel behavior framework (e.g., Van Acker et al., 2010), while beyond this thesis, would be a useful extension.

Also outside the scope of this theory is the extent to which day-to-day travel decisions influence these longer-term decisions. People who prefer to walk and cycle may choose (or select) to locate in a neighborhood that facilitates these active travel preferences. The self-selection influence has been noted in studies of land use and travel behavior (Cao, Mokhtarian, \& Handy, 2009; Handy et al., 2006; Mokhtarian \& Cao, 2008). If self-selection is not accounted for, estimates of the effect on travel behavior of land use and urban form policy and planning interventions will be biased and usually overestimated. The theory of travel decision-making addresses self-selection in a limited way through the inclusion of attitudes on perceptions of travel needs affordance. In addition, a simplified version of this travel decision-making process might be considered when people make locational or other longer-term decisions.

Another limitation is that the decision-maker implied by the theory may not be the same person as the traveler for whom decisions are being made. The very young and 
those who are physically or mentally incapacitated do not have conscious control over their mobility and must rely on parents or caretakers to both transport them and make their travel decisions. Thus, the theory of travel decision-making does not apply to these people without the ability to make their own travel decisions. Instead, the theory assumes some level of autonomy over one's travel decision-making.

That said, the travel decision-making process may actually apply to cases where control may not seem to rest with the individual traveler. Adults with physical disabilities that limit their ability to independently travel can arrange for others to transport them using paratransit, taxis, vans, or other means; their assessment of the hierarchy of travel needs may look very different from an able-bodied adult's. Even students who are escorted to primary or secondary school by parents or guardians may be considered to use this travel decision-making process. For example, even if a child's natural best outcome would be to walk while her parent's decision is to drive, the perception lens can explain why she ends up being driven to school. In children, subjective norms (how much she conforms to what she thinks her parents think she should do) are strong and self-efficacy (how confidence she is in walking to school) may be low. Thus, out of a dislike for disobeying her parents and a lack of confidence about being able to walk, the child may "submit" to being driven to school.

A final limitation is that the theory is only that: just a theory of travel behavior. Although it was developed after a thorough review of travel behavior and other theories from a number of fields and an in-depth investigation of empirical research documenting the influences on walking and bicycling, the components and relationships embodied by the theory of travel decision-making have yet to be tested, either in their specific form 
herein or as a collective group. Such an empirical analysis, using a single data source or consistent related data, is a logical and necessary next step if the theory is to be useful for making transportation planning and policy decisions related to walking and bicycling.

Despite these limitations, the theory of travel decision-making is both important and useful. It is one of a handful of conceptualizations of travel behavior influences and decision-making processes around which scholars have suggested designing research, tools, and policies (Handy, 2005; Saelens \& Handy, 2008). The theory can be used to guide any number of studies related to its hypothetical components and relationships. Its focus on active travel modes is particularly useful as officials, at least in the US, turn towards implementing policies designed to reduce car use and increase walking, cycling, and general physical activity. Yet, a valuable asset is the theory's broad appeal: it can apply to all modes of transportation. Thus, as will be elaborated in Chapter 4, the theory of travel decision-making can be used as a basis for reinventing the tools - namely regional travel demand forecasting models - by which transportation planning and related policy decisions are informed. 


\section{APPLYING THE THEORY TO TRAVEL DEMAND FORECASTING MODELS}

What lies ahead for disaggregate behavioral travel demand analysis? Between the extreme arguments that psychological elements are on one hand essential to understanding choice behavior and on the other hand impossible to incorporate into transportation planning models, where does the future lie? (McFadden, 2001, p. 37)

\subsection{Introduction}

Tools are valuable parts of transportation planning and policy-making processes. Transportation planning tools and their outputs inform decisions by politicians, officials, and the public about how to allocate billions of US dollars of infrastructure spending each year. Travel demand forecasting models - typically operated by metropolitan planning organizations (MPOs), large cities and transit agencies, and other regional and state governmental organizations - are among the most commonly used and institutionalized transportation planning tools. These travel models predict the anticipated demand for transportation facilities by different travel modes at some point in the future, among other things. They are rooted in a long history of travel demand modeling practice (Weiner, 2013), informed by travel survey data and travel behavior research. Although they are not the only transportation planning tools, nor the only applications of travel behavior research, travel demand models have great influence because they are institutionalized and widespread. 
Travel demand models have many planning applications, some of which are written into US law: prioritizing transportation projects in the short run $(\leq 4$ years), developing long-range transportation plans $(\geq 20$ years), supporting environmental impact statements for major transportation projects, evaluating proposed new major transit investments, and assuring conformity with air quality standards. Other applications include evaluating land use development changes, transportation system management and transportation demand management programs, environmental justice impacts, and traffic safety. With recent focus (e.g., MAP-21) on performance-based planning - using performance measures to prioritize transportation projects - the ability of travel models to forecast the necessary performance measures will become increasingly important. Travel demand models are being asked to do more to support transportation planning and policymaking processes.

While interest is increasing in many parts of the US about programs and investments to support and promote walking and bicycling and physical activity (e.g., Safe Routes to School, Complete Streets, Active Living, Green Lane Project), the travel forecasting models that support regional transportation investment decisions often do not or inadequately represent the demand for active travel modes. This is especially true when compared with travel model's abilities to represent the demand for auto and transit use. One section below reviews the state of travel forecasting models with respect to walking and cycling. Inadequate representation of active travel modes and of policies designed to affect their use in travel demand models, especially in an era of performance measurement using outputs from these tools, threatens to introduce bias into the transportation planning process. 
Moreover, the mathematical and statistical structures upon which basic operational travel demand models are based - particularly regarding influences on walking and bicycling - were historically developed atheoretically, based more on elegant (or inelegant, depending on one's perspective) fitting of empirical data. Models lack a strong derivation from travel behavior theory and often operate without due consideration of a human decision-making process. The focus of this thesis on developing a theory of travel decision-making that is applicable to the design of travel demand models is a direct reaction to this lack of theoretical and behavioral realism.

One of the most valuable applications of the theory of travel decision-making elucidated in the previous chapter is to reinvent the operation of travel demand forecasting models in a more behavioral manner in order to better-inform transportation planning and policy-making. The structuring of a travel model to accommodate all of the components and relationships of the theory should improve evaluations of all travel modes but particularly walking and bicycling. As will be noted in a later section, such a model could be used to evaluate transportation policies as wide-ranging as pedestrian and bicycle infrastructure investments, land use (re)development plans, evolving attitudes and preferences regarding active travel, and targeted informational and educational campaigns.

Incorporating all of these theoretical and conceptual aspects, including the hierarchy of travel needs and the mediated moderation of demographics, socioeconomics, and perceptions, is no simple task. Recent efforts to model travel demand as activitybased have yielded some improvements upon basic trip-based models, but significant challenges remain. For example, some, including McFadden (2001), have questioned 
whether social-psychological factors could or even should be integrated into travel modeling. The necessary data and analytical methods to structure those data must be available and understood in order for such an integration to be possible; they might also need to be simply operationalized for these methods to become commonplace. A prior requirement is for travel behavior research to document the conceptual components and relationships suggested by the theory.

Table 4-1 summarizes the content of the proceeding sections. Each of the components and relationships expressed by the theory of travel decision-making have been ranked across three aspects: 1) how they are currently present in travel demand forecasting models; 2) how they are supported by travel behavior research; and 3) how available are their respective data and analysis methods. Note that this summary of the state of the practice has yet to be externally validated, and others might disagree with the rankings. Some aspects of the theory are well-documented across all three dimensions, while several others are poorly done. What is most important to consider is the relative ranking of components/relationships within each category; of course more work can be done even in "well-represented" areas. This analysis informs future work to research and apply the theory to practical travel demand modeling. 
Table 4-1: State of the Theory of Travel Decision-Making with Respect to Travel

Forecasting and Modeling, Travel Behavior Research, and Data and Analysis

Methods

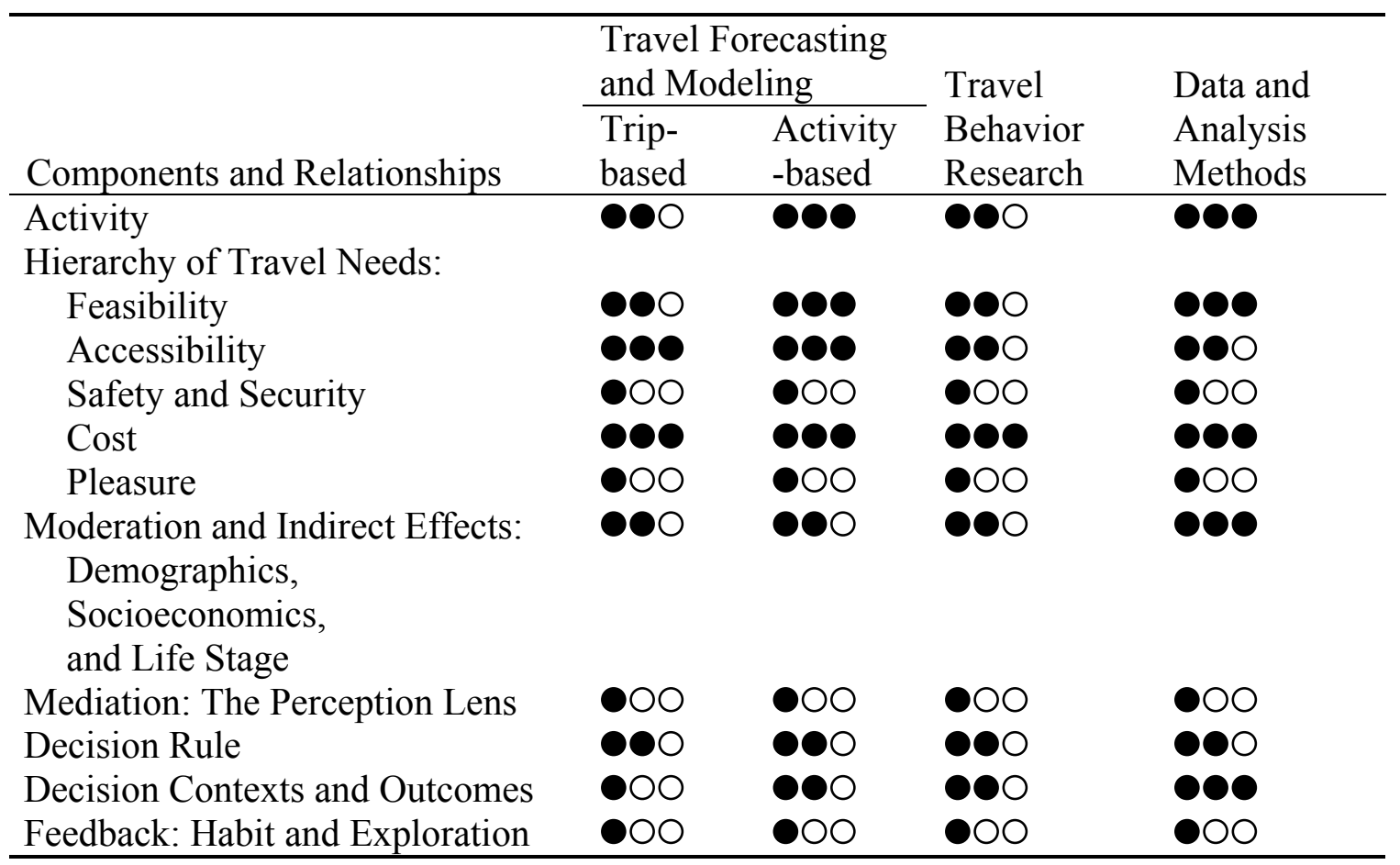

00: included or well-represented;

००: partially included or somewhat represented;

००: not included or poorly represented.

The following sections provide detail of the assessments summarized by Table 4-1. First, the state of travel forecasting is presented, with a summary review of walking and cycling in travel demand models. Next, the state of travel behavior research in support of the theory is summarized. Third, the availability of data and methods of analysis for each aspect are described. The chapter closes with a discussion of opportunities for future integration and application of the theory of travel decisionmaking into travel demand forecasting models. 


\subsection{State of Travel Forecasting}

The following section describes the state of travel forecasting and demand modeling, with a particular focus on walking and bicycling, with respect to the components and relationships in the theory of travel decision-making. One of the major applications of this theory is its utility in explaining what factors and relationships should be operationalized within travel models. After an overview of how travel demand models operate and a review of the current state of the practice of representing walking and cycling in these forecasting tools, each part of the theory is discussed.

\subsubsection{Overview of Travel Demand Modeling}

Travel demand forecasting models use mathematical equations - based on statistical data analysis techniques and observed travel behavior - and basic data inputs population and employment totals, scenarios of land use and transportation supplies - to make predictions of future travel demand in a region. These travel demand estimates are often as detailed as the number of trips (a journey from an origin to a destination) by mode, time-of-day, neighborhood, or even facility segments and intersections. The reliability of models depends on their operational framework, the validity of the equations within them, and the underlying estimation and input data.

Historically, travel demand models have utilized a sequence of four key stages for forecasting personal transportation, the movement of people (as opposed to freight transportation, the movement of goods):

- Trip generation: How many trips begin or end here?

- Trip distribution: Where do those trips go, or where did they come from? 
- Mode choice: By what mode (auto, transit, walk, bike) do these trips travel?

- Trip assignment: By which routes do these trips travel?

Trips are also frequently distinguished by time-of-day, auto occupancy, and income group of traveler. Advanced travel demand modeling systems often operate more like simulation models, tracking households and individuals through the whole process, predicting activity patterns, structuring trips into round-trip tours, and integrating travel models with models of land use development and other long-term economic and locational decisions.

\subsubsection{Walking and Cycling in Travel Demand Forecasting Models}

Transportation planning tools, particularly travel demand forecasting models, are being asked to provide input into many new areas of concern, including air quality, climate change, energy and environmental sustainability, public health, and equity (Handy, 2008). In response, many US metropolitan planning organizations (MPOs) have begun to include or are expanding the representation of walking and bicycling modes in their travel demand models. Such models can then be used for assessing pedestrian and bicycle infrastructure investments, developing active transportation plans, conducting health impact assessments, and other planning efforts.

Many reviews have documented this evolution in practice over the last two decades (Cambridge Systematics \& Barton Aschman, 1994; Committee for Determination of the State of the Practice in Metropolitan Area Travel Forecasting, 2007; Eash, 1997, 1999; Liu, Evans, \& Rossi, 2012; Nourzad, 2000; Porter, Suhrbier, \& Schwartz, 1999; Purvis, 1997; Replogle, 1997; Rossi, 2000; Vanasse Hangen Brustlin, 
2007). A recent review of the state-of-the-practice (Singleton \& Clifton, 2013) comprehensively documented how MPO models represent active travel modes (with a focus on walking). Highlights and key points of the review are summarized below.

The first documented regional travel demand model to include walking or bicycling travel was a binary logit non-motorized mode split model developed in 1988 at the Metropolitan Service District (now Metro) of Portland, Oregon (Purvis, 1997). At around the same time, the Maryland-National Capital Park and Planning Commission developed the first non-motorized-specific built environment measure: the pedestrian and bicycle friendliness index (Cambridge Systematics \& Barton Aschman, 1994; Replogle, 1997). Another influential project at Metro in the mid-1990s introduced the pedestrian environment factor: an index of the ease of street crossings, sidewalk continuity, grid street pattern, and terrain to be applied in a pre-mode choice non-motorized split model (Cambridge Systematics \& Parsons, Brinckerhoff, Quade \& Douglas, 1996; Cambridge Systematics, et al., 1996; Parsons Brinckerhoff Quade and Douglas, Cambridge Systematics, \& Calthorpe, 1993). Through the next two decades, more regions continued to add non-motorized, walking, and/or bicycling modes to their travel demand forecasting models.

A review of the travel models used by the 48 largest MPOs (Transportation Planning Capacity Building Program, n.d.) uncovered several ways in which walking and bicycling are represented (Singleton \& Clifton, 2013). In four-step trip-based models, non-motorized trips can be generated on their own, separated from motorized trips before or after distribution, distinguished from trips of other modes during mode choice, or separated into walking and bicycling trips. Calculated trips by active modes are output 
and usually not included in downstream modeling stages. Similar frameworks are used to represent active travel in tour- and activity-based models (ABMs). At their heart, ABMs use many of the same RUM-based model structures as trip-based models; therefore, they can be described analogously (e.g., tour generation, number of stops, destination choice, tour and trip mode choice). Figure 4-1 graphically represents these different active travel modeling frameworks.

Most (30 or $63 \%$ ) of the largest 48 MPOs model active travel, of which almost half (14 or $47 \%$ ) distinguish between walk and bicycle trips within mode choice. Two agencies apply framework 1 with a parallel non-motorized trip generation process. Five agencies use each of frameworks 2 and 3 in which non-motorized trips are split from motorized trips prior to a full mode choice model. Eighteen agencies include active travel mode alternatives within the mode choice stage, framework 4. Only a handful of agencies, including transportation planning organizations representing the Portland, Oregon, region and San Francisco County, assign bicycle or pedestrian trips to the network (Bomberg et al., 2013; Broach et al., 2012; Hood et al., 2011; Stein, 2011; Zorn, Sall, \& Bomberg, 2012), framework 5. 
(0)

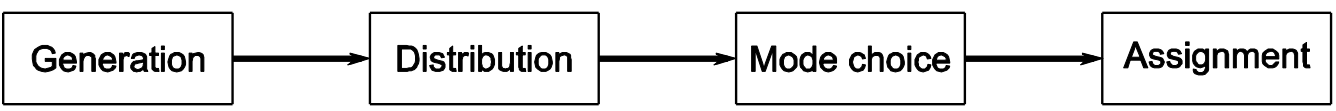

(1)

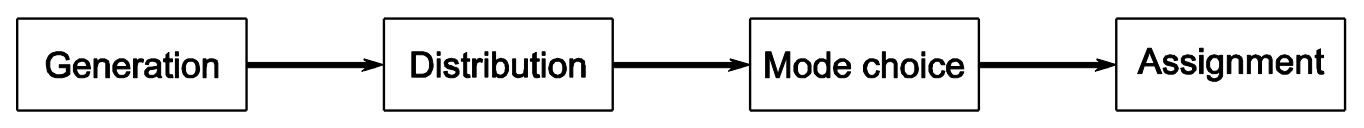

Generation

$\dot{\lambda}$ Sio Productions \& attractions

(2)

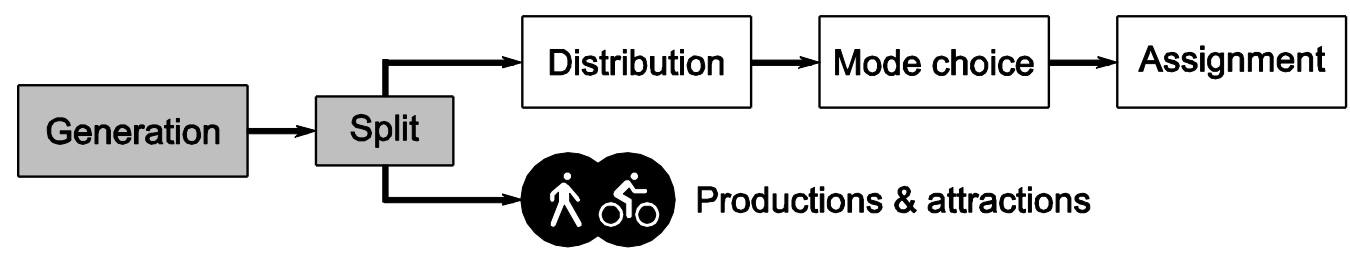

(3)

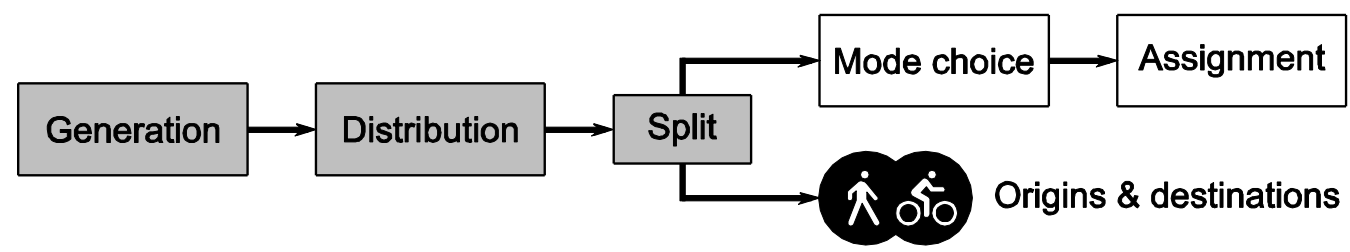

(4)

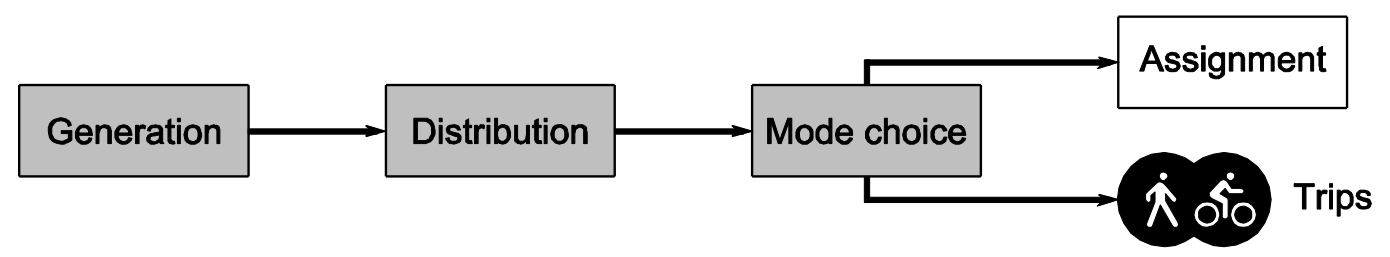

(5)

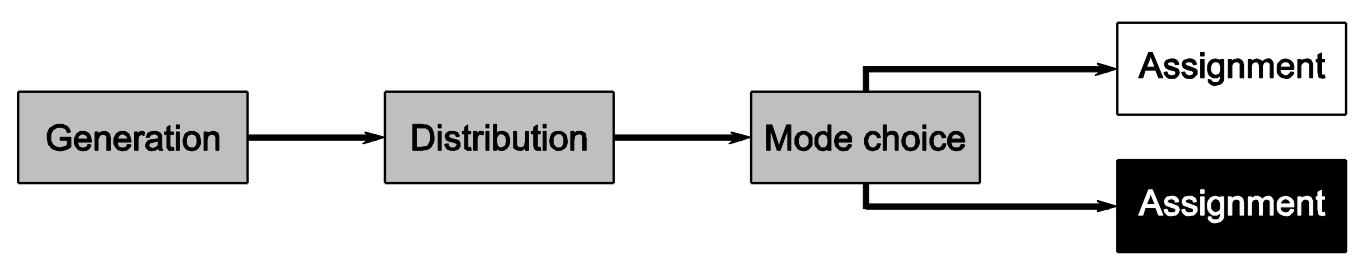

Legend

\# Modeling framework

i

Auto, transit

\section{All modes}

Walk, bicycle

Model stage, modes

Figure 4-1: Active Travel Modeling Frameworks

(Singleton \& Clifton, 2013) 
A number of different variables, mathematical structures, and parameters are used to include walking and bicycling in regional travel demand models. Some of the most common factors are: level-of-service variables (used in 95\% of relevant models), including trip distance and travel time; demographic and socioeconomic variables (used in $88 \%$ of models), including household size, income, and vehicle ownership; and environmental density variables (used in $85 \%$ of models), including residential density, employment density, and area type. The majority of models utilizing frameworks 2, 3, and 4 apply binary, multinomial, or nested logit model structures to represent the choice between travel modes. Travel times are calculated based on assumed speeds: 2.5 to 3.0 mph for walking, 7 to $12 \mathrm{mph}$ for cycling. Another frequent practice is to prohibit long walk and bicycle trips; common walk trip limits are 3 or 5 miles, while bicycle maximums vary from 6 to 20 miles (Singleton \& Clifton, 2013).

Innovative practices are emerging that have the potential to improve upon some limitations in how regional travel demand models represent walking and bicycling. More effective survey design approaches can reduce the underreporting of non-motorized and multimodal trips (Clifton \& Muhs, 2012). Planning organizations in some regions are able to gather and include more fine-grained measures of the relevant street-level environment, including grade, sidewalk availability, bicycle facility types, roadway conditions, and specific types of businesses (Johnson Gardner, 2007). A number of large MPOs are using different, usually smaller, spatial units and more complete networks for walking and bicycling analysis. There is renewed interest in walking and cycling route choice with the emergence of GPS-based travel surveys. 
An understanding of the current state of the practice with regard to walking and bicycling in travel forecasting tools is useful. This information provides insight into how aspects of the theory of (active) travel decision-making are currently operationalized or might be incorporated in the future.

\subsubsection{Current Travel Model Operationalization of the Theory}

Two major categories of regional travel models are currently in operation: (fourstep) trip-based models (TBMs) and (tour- or) activity-based models (ABMs). Figure 4-2 and Figure 4-3 show how the components and relationships in the theory of travel decision-making are addressed within trip- and activity-based models, respectively. The following sections discuss in more detail how current models in practice fit within this theoretical framework and how each aspect of the theory is currently accommodated and operationalized or ignored. 


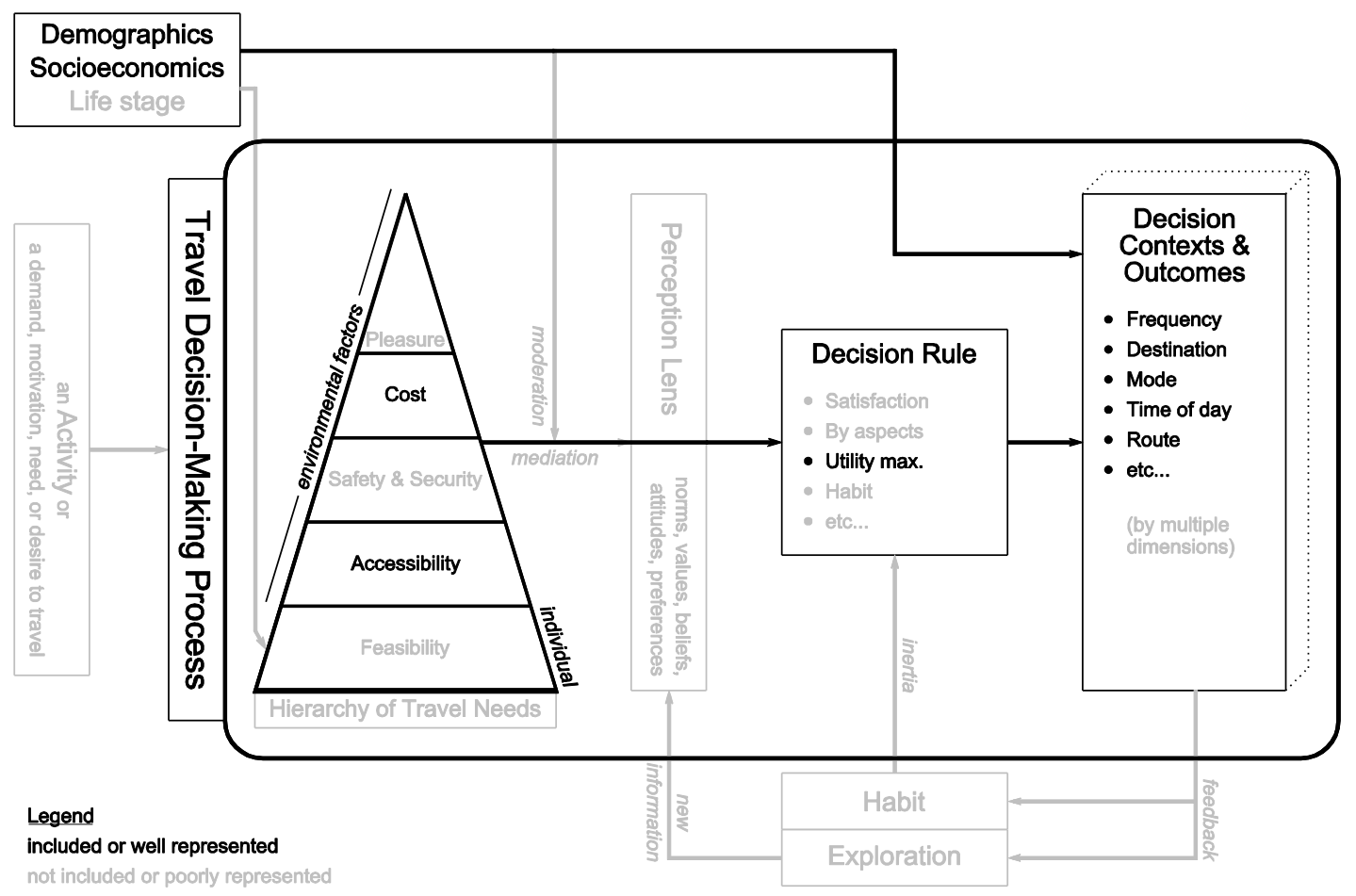

Figure 4-2: Trip-Based Models in the Theory of Travel Decision-Making

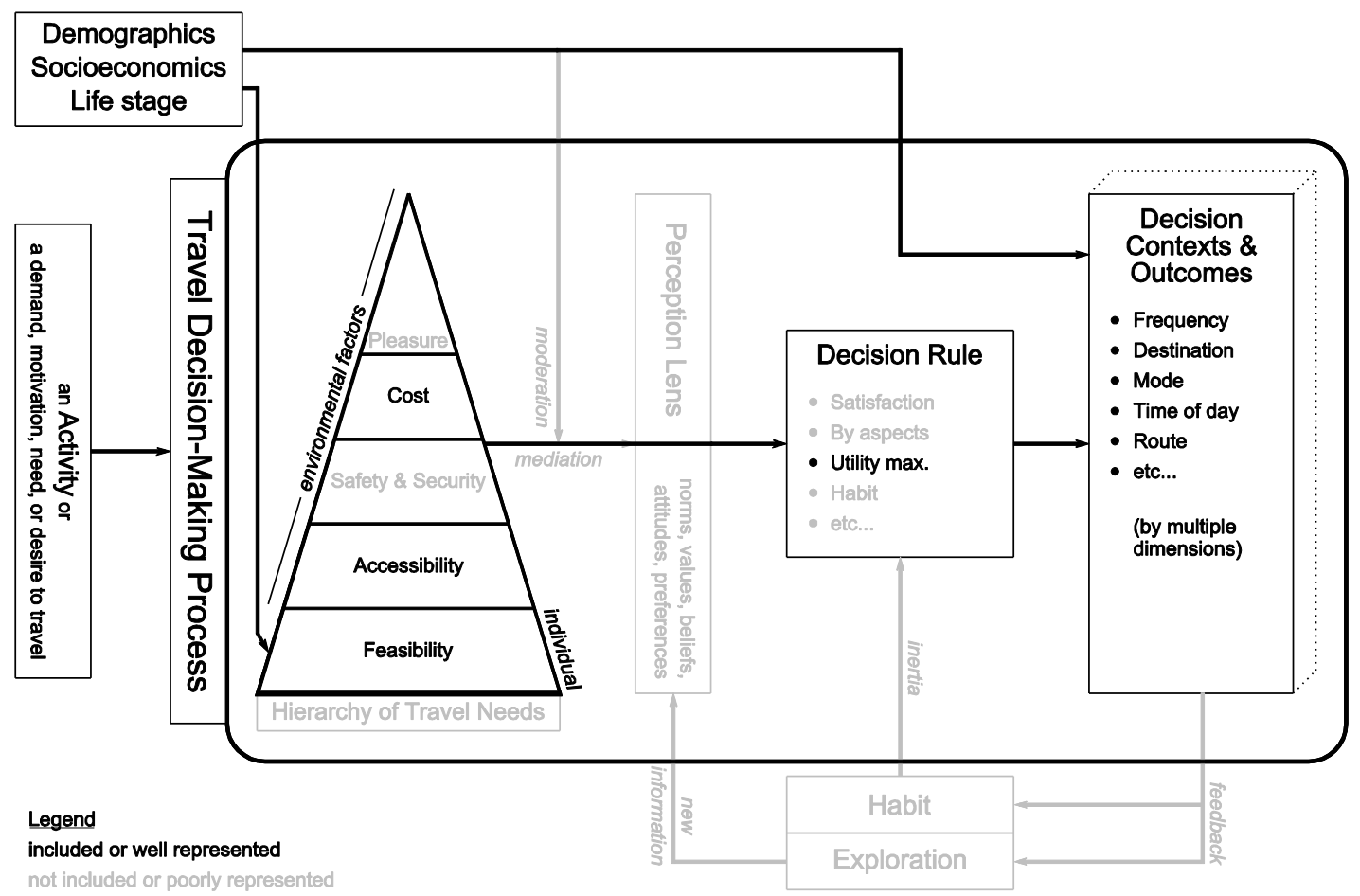

Figure 4-3: Activity-Based Models in the Theory of Travel Decision-Making 


\subsubsection{Activity}

Representing travel as a demand derived from activity generation is only partially done in TBMs. To the extent that trip generation models are segmented by trip purpose home-based work, home-based non-work, and non-home-based purposes are common there is some accounting for very rough categorizations of the demand for different activity types. On the other hand, ABMs were designed with exactly this purpose in mind. The selection of daily activity patterns, home- and work-based tours, and primary and additional tour activities allows for an explicit representation of activity prioritization and how activities lead to and constrain travel (Ortúzar \& Willumsen, 2011). Usually, more activity types are considered in activity-based models than in trip-based models.

\subsubsection{Hierarchy of Travel Needs}

\subsubsection{2.a. Feasibility}

Basic four-step trip-based travel demand forecasting models deal with feasibility in a rough and incomplete way. To the extent that individual or household variables are included that affect feasibility, the strongest influence is usually auto ownership or availability, higher levels of which have a negative influence on the propensity to walk or a positive influence on the propensity to drive. Other household variables - household size, number of children or workers - play a largely demographic role in influencing travel decisions rather than defining the feasibility of those options. Some models do restrict certain travel modes from being chosen in certain situations: walk and bicycle modes are not options for trips longer than a set distance (3-15 miles), or walk-to-transit 
is not an option if there is no transit stop within a small distance (1/4-1 mile). Due to the primarily aggregate application of TBMs, they have virtually no treatment of time-space or other scheduling constraints.

On the other hand, defining feasibility is where activity-based models shine over trip-based models. Heavily informed by Hägerstrand's time-space prism (1970) and Chapin's description of activities motivating travel (1974), ABMs typically excel at representing the activity-scheduling constraints that define the feasibility of travel choices. Primary tours are scheduled prior to secondary tours and intermediate stops, which are constrained by those prior - assumed to be more important or fixed decisions. Because of the explicit consideration of households and individuals in ABMs, intra-household feasibility constraints imposed by vehicle use or escorting needs can be accommodated. Finally, trip mode choices are logically constrained by the choice of tour mode (Ben-Akiva \& Bowman, 1998a; Ortúzar \& Willumsen, 2011).

Activity-based models present a more compelling and realistic way to represent feasibility within the hierarchy or travel needs than trip-based models, although possibilities exist for further improvements. Notably, feasibility is mostly imposed $a$ priori by the modeler through rules and sequences: some modes are unavailable for trips based on the chosen tour mode, and intermediate tour stops are chosen prior to trip mode. Also, there is no way to include variations in knowledge or awareness of travel alternatives. 


\subsubsection{2.b. Accessibility}

Trip-based models do incorporate many environmental measures related to accessibility. Density or proximity variables are included in a number of ways. Trip generation cross-classification models often segment productions not only by trip purpose by also by area type, a categorization of urban form usually determined by a combination of residential and employment densities. The local density of jobs is a key factor in trip distribution gravity models (implicitly through trip attractions) and in destination choice models. Residential and employment density often enter walk and bicycle mode choice utility equations as well (Cambridge Systematics et al., 2012).

Connectivity measures are also used for TBMs in frameworks 2, 3, and 4 but less frequently than density measures. Intersection density (or its inverse, block density) is the most common variable, but measures of average block size and non-motorized path density are also used in a MPO travel model covering the Raleigh area. Newark's MPO applies unique measures of network connectivity (\# intersections / total street distance), and network restrictivity (\% roadway network where pedestrians are prohibited). Some simple diversity measures, distinguishing between residential-only and job-producing land uses, occasionally enter mode choice model utility equations (Singleton \& Clifton, 2013).

Activity-based models also incorporate many environmental measures of the accessibility need in much the same way as TBMs, although most ABMs distinguish walking and bicycling from motorized travel within the tour and trip mode choice stages. A recent trend among $\mathrm{ABMs}$ and even some TBMs is to include all built environment factors within one accessibility or mix variable: usually a function of households, jobs, 
and sometimes intersections. These variables increase with both development density and land use diversity, allowing both of these highly-correlated measures to impact modeled travel choices (Reiff \& Kim, 2003).

\subsubsection{2.c. Safety and Security}

All trip-based and activity-based models do not yet explicitly consider traffic safety or personal security concerns as a basis for making a travel choice, such as between walking, bicycling, and other modes. Traffic safety for walking and bicycling

mode choice is occasionally implicitly and incompletely addressed when measures of roadway characteristics are included as variables, such as the local density of freeways, sidewalks, or multi-use paths, or in the "ease of crossing streets" dimension of the PEF. Bicycle facility types are important variables in bicycle route choice and assignment, where implemented (Broach et al., 2012; Hood et al., 2011). Yet, such safety proxy variables are rarely included in travel models. To the author's knowledge, no travel forecasting model has attempted to include actual or perceived crime rates or other personal security measures.

\subsubsection{2.d. Cost}

Travel demand forecasting models, whether TBMs or ABMs, do the best job of representing cost within the hierarchy of travel needs. Nearly all MPO models include either a distance, time, generalized cost, or other level-of-service variable in the (tour or trip) mode choice utility equations for walking and bicycling when such modes are 
represented (Singleton \& Clifton, 2013). While monetary costs are included for other modes (auto and transit), walking and cycling are typically free.

Some improvements could occur with how costs are calculated for active travel. Most estimates of walking or cycling travel time are taken from distance-based zone-tozone skims along the street network, where a single speed has been assumed. Allowing these speeds to vary based on individual characteristics (age and gender) or by grade, particularly for cycling, might produce more accurate travel time estimates. Accuracy may also increase by making zones smaller or using parcels and by estimating time using a full network of sidewalks, bicycle facilities, and off-street paths. Some MPOs, including one in Sacramento, are starting to implement such spatial improvements in their models. Activity-based models tend to be better suited than trip-based models to produce more accurate estimates of travel time due to their frequent use of smaller zones and more detailed walking and cycling networks.

Other cost factors are just beginning to be used in operational travel models. Physical effort has been captured in some recent bicycle route choice models (Broach et al., 2012; Hood et al., 2011) and one tour mode choice model with path-based environment characteristics (Bomberg et al., 2013). In these applications, topographical measures of terrain, including total rise and percentage upslope of grade, represent negative influences of physical exertion on the choice of walking or of a cycling route.

While travel time reliability is rarely considered by travel forecasting models, recent work (Brennard, 2011; Parsons Brinkerhoff, 2012; Vovsha, 2012) has begun to identify ways to include reliability into traffic assignment models, particularly dynamic traffic assignment (DTA). Travel time reliability is an important part of the second 
Strategic Highway Research Program (SHRP 2), especially projects C04 and L04 which dealt with modeling. Using reliability measures in DTA holds special promise when combined with ABMs (Parsons Brinkerhoff, 2012). However, such efforts are currently limited to travel time reliability on highways and have yet to consider walking and cycling under congested conditions.

\subsubsection{2.e. $\quad$ Pleasure}

Similarly to the safety and security need, both TBMs and ABMs do not include factors that would contribute towards the pleasure need. Environmental variables are nearly always limited to those that define the accessibility need: density, diversity, and connectivity. One exception are measures of terrain/grade/topography included in bicycle route choice models (Broach et al., 2012; Hood et al., 2011) and one tour mode choice model that includes path-based environmental characteristics (Bomberg et al., 2013). While this variable tends to have a negative influence on the choice of walking or of a cycling route, indicating it acts primarily as the cost of physical exertion, some smaller amount within the parameter estimate likely accounts for a minor benefit of physical activity. Other factors in the pleasure need, including comfort, aesthetics, and mental and social enjoyment, are only implicitly captured in mode-specific constants estimated for mode choice models.

\subsubsection{Moderation and Indirect Effects}

Both categories of operational travel models - TBMs and ABMs - include individual characteristics variables that influence the feasibility of travel options, usually 
in the form of auto ownership or sufficiency (\# autos per person or worker) measures. Moderation by demographic and socioeconomic factors is sometimes included through interaction terms and market segmentation. For instance, in TBMs, trip generation rates are often classified by income category and/or other household characteristics like size, number of workers, or number of children. Destination and mode choice factors frequently cost or constants - are sometimes estimated with different coefficients based on income or auto ownership categories (Cambridge Systematics et al., 2012). ABMs, by maintaining personal characteristics in all activity-, tour-, and trip-making, are able to more realistically represent demographic and socioeconomic characteristics of travel (Donnelly, Erhardt, Moeckel, \& Davidson, 2010). ABM stages also sometimes include interactions of choice model cost or constant variables with income or various demographic factors. These interaction effects are more commonly applied to auto and transit modes than for walking and bicycling.

Both trip- and activity-based models also sometimes include demographic and socioeconomic characteristics variables directly into mode choice utility equations. Common traveler characteristics include the afore-mentioned auto ownership and household income variables, as well as gender, age, and worker/student status (Cambridge Systematics et al., 2012). It may not be well understood that these variables are not expected to be directly causal. Instead, these demographic and socioeconomic variables included without interaction are meant to substitute for average variations in unobserved attributes. For example, there is likely not something inherent in US men that makes them more likely to choose cycling. Instead, it is more likely that household obligations, the current state of bicycle facilities, perceptions of safety provided by those 
facilities, and other social factors means that people cycling in the US today are more likely to be male. It is precisely these unobserved attributes that the theory is trying to uncover through perceptions of how much a travel alternative fulfills the hierarchy of travel needs.

\subsubsection{Mediation}

Perceptions of travel needs fulfillment are not explicitly accounted for in today's operational travel demand forecasting models. Aggregate trends in attitudes and perceptions may be implicitly included in mode choice models via demographic and socioeconomic variables and mode-specific constant terms. These methods of representing travel perceptions are incomplete if decision-makers want to predict the travel changes as a result of making non-environmental interventions such as education or awareness programs. A promising area of future work should investigate methods to include these perceptive factors into operational transportation planning tools.

\subsubsection{Decision Rule}

Most trip-based and nearly all activity-based models represent travel decision processes as discrete choice problems, based in random utility maximization frameworks. These RUM-based structures include binary logit, multinomial logit, nested logit, and minor variations thereof. Some TBM stages, including trip generation and distribution, frequently deal with aggregate zonal travel using non-behavioral structures like crossclassification tables and the gravity model (Cambridge Systematics et al., 2012). Some TBM structures impose decision rules a priori: walking cannot be chosen for trips greater 
than a certain length. Some ABM structures also impose decision rules a priori: if walking was chosen for the tour mode, only walking can be chosen for all trips on that tour. Other non-compensatory decision rules have been proposed and prototyped (Bowman \& Ben-Akiva, 1997; Gärling et al., 1994; McNally \& Rindt, 2008), including STARCHILD (Recker et al., 1986a, 1986b), AMOS (RDC, 1995), and ALBATROSS (Arentze, Hofman, van Mourik, \& Timmermans, 2000), yet no operational US travel demand forecasting model with these alternative decision rules has emerged.

\subsubsection{Decision Contexts and Outcomes}

TBMs typically model a limited number of travel decision contexts: trip generation, trip distribution (destination choice), mode choice, traffic assignment (route choice), and sometimes time-of-day choice. These choices are usually modeled sequentially, with limited feedback (Cambridge Systematics et al., 2012). On the other hand, ABMs typically model a much larger number of travel choices due to their activityand tour-based foci: day activity pattern, joint travel, primary activity purpose and location, time-of-day, tour mode choice, intermediate stops, trip mode choice, etc. (Donnelly et al., 2010). These choices are modeled sequentially or conditionally, with frequent feedback. Feedback mechanisms - logsums from lower level models used as variables in higher level models, for instance - partially represent the interrelatedness of travel choices across multiple dimensions (Freedman \& Goulias, 2012). 


\subsubsection{Feedback}

Feedback mechanisms of the form envisioned by the theory of travel decisionmaking - habit and exploration or new information - do not yet exist in trip- or activitybased models. Although feedback loops are present in some TBMs and most ABMs, they represent a different set of processes. As mentioned in the previous section, when components of lower level model stages are used as input variables for higher level models - such as when mode choice logsums are used for destination choice - the feedback reflects the sequential or conditional nature of the modeled choice process and represents some of the interdependencies between different travel choice dimensions. In ABMs, when destination and mode choice logsums are used as variables in models of long-term choices such as work location, the feedback mechanism attempts to get at the role that expected travel has on travel-related locational choices. Past travel choices, beyond the extent to which they are expressed by residential location and vehicle ownership, do not currently enter as variables in regional travel models. Operational models also cannot represent the impact that new information is hypothesized to have on awareness and perceptions of travel alternatives.

\subsubsection{Summary}

Existing trip- and activity-based travel demand forecasting models are able to represent several aspects of the theory of travel decision-making, including the cost and accessibility needs. Activities, the feasibility need, and moderation by traveler characteristics are also accommodated to a lesser extent. Because of their greater complexity, ABM systems more closely incorporate aspects of the theory, particularly for 
walking and bicycling. Some aspects of the theory are not accommodated at all, including the safety/security and pleasure needs, the perception lens, and the influences of habit and exploration. As will be discussed in the following sections, there are many reasons why travel models do not more closely align with the theory, including limited knowledge from travel behavior research and a lack of sufficient data and analysis methods.

\subsection{State of Travel Behavior Research}

For a travel demand modeling framework to be relevant and useful in real-world applications, the relationships within it must find empirical support from travel behavior research. It is imperative to know what factors have been shown to influence walking and bicycling and the form of those relationships so that appropriate data can be collected and modeling techniques used. While Chapter 3 contains much of theoretical and empirical justifications for the components and relationships contained in the theory of travel decision-making, this section reviews the state of travel behavior research and notes places for improvements and future work. Table 4-1 summarizes these conclusions.

Most scholars agree that an activity-based approach to modeling travel is, at least theoretically, superior and more realistic than a trip-based approach (McNally \& Rindt, 2008; Ortúzar \& Willumsen, 2011). Recent research has investigated activity patterns (Kuppam \& Pendyala, 2001; Ma \& Goulias, 1997) and the temporal and spatial distribution of activity participation (Gliebe \& Kim, 2010; Kwan \& Lee, 2003). More work is needed in a number of areas, including examining the interactions between activities and travel, particularly how activity demand may vary based on mode and other choices. 
The field's knowledge about the attributes and influences of the hierarchy of travel needs varies considerably. The cost need likely has the most consistent understanding in travel behavior literature. Travel time and monetary cost are usually among the biggest drivers in travel choices. While less attention has been paid to physical effort, mental effort, convenience, and reliability, these factors likely have only marginal effects compared to time and cost. The feasibility need is also relatively well documented in travel behavior literature. The influence of mode availability and individual mobility factors has long been acknowledged (Quarmby, 1967). More recently, the impact of activity and travel schedules has seen increasing interest (Akar, 2009). More work is needed investigating the impact of inter- and intra-household household interactions on active travel behavior.

A considerable and impressive body of knowledge has emerged in recent decades on the impact of environmental factors, especially accessibility, on travel behavior. The impact of the location and proximity of destinations on walking and bicycling appears to be clear: at least walking is more likely with higher residential and employment densities and closer destinations (Saelens \& Handy, 2008). Reviews also consistently find positive associations between land use mix or diversity and active travel (Ewing \& Cervero, 2010; Saelens \& Handy, 2008). Street and sidewalk connectivity also appears to facilitate walking and bicycling (Berrigan et al., 2010). Recently, attention has shifted from zonal and areal buffers to path-based measures of accessibility. Future research should investigate better measures of diversity and connectivity, test the impact of path-based accessibility measures, and examine the effects of accessibility on bicycling. 
Safety and security effects on active travel are poorly understood. Negative perceptions of traffic safety do appear to reduce children's active travel and people cycling prefer routes with less and slower traffic and greater separation, yet traffic safety has inconclusive results in other contexts and for adults. The effect of fears of crime and personal security concerns are even more equivocal and seem to apply more to walking than to cycling. Finally, the pleasure or enjoyment need has received probably the least attention among all five needs in the hierarchy. The impact of comfort, exercise, aesthetics, and travel liking are rarely examined, and significant effects (when found) tend to be small. More work is needed in these areas.

Some empirical evidence supports the hypothesis that demographic, socioeconomic, and life stage characteristics moderate the relationship between environmental characteristics and active travel behavior, particularly for differences by gender and age (Saelens \& Handy, 2008). Other individual characteristics are likely moderators, but few studies test for them and their effects may be difficult to detect. Recent attention has also found some reason to support the hypothesis that perceptions mediate the relationship of aspects of the hierarchy of travel needs on travel behavior. Attitudes towards travel modes, normative influences, and self-efficacy have been found to affect travel mode choice (Bamberg, Rölle, \& Weber, 2003). There seems to be little consistency on how such perceptive aspects are tested in relation to travel, nor how they are placed within a larger behavioral framework. More work is needed specifically testing perceptions of the five components of the hierarchy of travel needs. In addition, few studies in travel behavior have investigated the integrated "mediated-moderation" hypothesis presented in the theory of travel decision-making. 
Considering the empirical success that a decision rule based in utility maximization has had in explaining relationships with travel behavior, it is understandable that less attention has been paid to uncovering the actual decision rules used in travel choices. Future work might investigate the potential application of noncompensatory decision rules, especially for mode choice, even if such work utilizes RUM-based discrete choice analysis methods (Gilbride \& Allenby, 2004). On a related note, all sorts of travel choices have been studied in depth, including trip generation and destination, mode, and route choices. Much less work has investigated the possibility that such choices happen jointly or in a different sequential order than is commonly assumed.

Some evidence exists to support the presumption that habits and new information can affect the travel decision-making process. Habitual travel behavior appears to reduce the evaluation of travel needs fulfillment, and interventions appear to be able to change individuals' perceptions and awareness, although much of this research focuses on changes in car use (Bamberg, Rölle, \& Weber, 2003; Klöckner et al., 2003). Much more work is needed in this area to see how and when habits and new information come into play.

To summarize, the state of travel behavior research strongly supports some aspects of the theory of travel decision-making while providing mixed or marginal support for other components. Overall, most aspects are adequately supported, at least enough to build a theoretical framework and apply it to travel demand modeling systems. Research gaps tend to be where theory provides a less firm footing and where relationships, if they exist, are weaker or moderated/mediated by other factors. These areas are prime for future theoretical development and empirical research. 


\subsection{State of Data and Analysis Methods}

Another important requirement for an operational travel demand forecasting model is the data and analysis methods capable of representing findings from travel behavior research. Even if a relationship has been found to be significant in academic literature, it will have no bearing on travel models (and decisions based upon their results) unless the necessary data can be measured and/or collected and an appropriate analysis method developed. Both requirements must also work within the travel model system; that is, necessary data must fit within the data collection or synthetic data generation process, and relationships must be able to be expressed mathematically within the sequence of travel model steps. This section describes the current state of data collection for each of the components in the theory of travel decision-making, and the current state of analytical methods available to formulate each of the relationships in the theory.

\subsubsection{Current Data and Methods for the Theory}

\subsubsection{Activity}

The methods for collecting data on the activity basis of travel are continually improving. Regional, statewide, and national household travel surveys, as well as specialized transit on-board and campus surveys, are typically used to estimate and calibrate regional travel demand forecasting models. Many of these surveys have begun to shift from simply accounting for trip-making to focusing on activities conducted as well as personal transportation to and from those activities (Stopher, 1992). These 
improved household travel and activity surveys, along with discrete choice methods for selecting activity patterns, allow for a much stronger way - at least in activity-based models - for representing that travel demand is generally derived from a demand for activity participation.

\subsubsection{Hierarchy of Travel Needs}

\subsubsection{2.a. Feasibility}

Within the hierarchy of travel needs, factors comprising the feasibility need are relatively well documented and available for use in travel demand forecasting models. Recent household travel surveys frequently ask about these factors, including questions about physical disabilities that inform individual mobility. Mode availability is also regularly collected with questions on the number of autos and bicycles owned, the holding of transit passes and drivers licenses, and even membership in vehicle-sharing services (Federal Highway Administration, 2011; Oregon Modeling Steering Committee, 2011). Geographic information systems (GIS) allow for the relation of activity locations and public transportation service to define transit feasibility. Future travel surveys might also inquire whether other non-chosen modes were considered feasible alternatives for particular trips to get a sense of perceived feasibility.

Individual schedules and intra-household interactions are also normally captured in recent household travel surveys, which inquire about vehicles used and other travelers on the journey (Metropolitan Washington Council of Governments, 2012; Oregon Modeling Steering Committee, 2012). These data allow for coupling constraints to be 
imposed among people and vehicles in both time and space. Activity-based models are particularly well-designed to accommodate and simulate activity scheduling and household interactions. More research could illuminate the extent to which scheduled activities are flexible and the degree to which people jointly coordinate activities and travel.

\subsubsection{2.b. Accessibility}

Travel demand forecasting models do a moderately good job of representing the theory's accessibility need in a large part because accessibility-related data are available. The most commonly-used measures of proximity to destinations - residential and employment densities - can be calculated using household and business location data usually available to MPOs across an entire metropolitan area, often at small spatial scale such as parcels. Land use data used in measures of diversity or land use mix can often also be obtained from most jurisdictions. However, there are a wide range of possible land use diversity measures with little consensus about the "ideal" mix for walking and bicycling (Gehrke, 2012).

A similar problem arises for measures of network connectivity. Basic measures such as intersection and block densities can be calculated (at least in the US) from roadway GIS databases such as Tiger/Line ${ }^{\circledR}$ shapefiles from the US Census Bureau (US Census Bureau, 2013). More detailed spatial information on walk- and bicycle-specific networks, including sidewalks, off-street paths, and bicycle facility locations and types, may be incomplete or unavailable for the entire travel model region. In addition, there are a number of different connectivity measures, again with little consensus about which 
ones are most strongly related to active travel (Berrigan et al., 2010; Dill, 2004; Tal \& Handy, 2012).

Path-based accessibility characteristics are even more difficult to measure. Even if detailed parcel-level land use and urban design data are available, these path-based measures require information on actual or expected walking and bicycling routes. With the exception of emerging GPS-based surveys, typical household travel surveys do not capture the routes along which walking and bicycling occur. Furthermore, the ability to simulate preferred walking and cycling routes using route choice models is still in its infancy, despite recent efforts (Bomberg et al., 2013; Broach et al., 2012; Hood et al., 2011; Stein, 2011; Zorn et al., 2012).

A number of other considerations combine to limit the effectiveness of current data and methods for calculating and applying accessibility measures in travel demand models (Iacono, Krizek, \& El-Geneidy, 2010). In addition to the data limitations noted above, many current travel models impose operational limits on spatial scales. The typical units of analysis, transportation analysis zones (TAZs), tend to be so large outside of the central city that they obscure variations in urban development intensity, increasing the likelihood of an ecological fallacy. A related issue is the use of coarse network structures which typically include those roadways least desirable for active travel and require significant effort to calculate connectivity and path-based accessibility measures. Several MPOs are breaking these limits by using smaller analysis zones, developing specialized walk and bicycle networks, and measuring more detailed information about urban street walking and cycling facilities (Singleton \& Clifton, 2013). 


\subsubsection{2.c. Safety and Security}

To the author's knowledge, no travel demand forecasting model has attempted to include either objective or perceived measures of security from crime. Data on traffic safety for walking and bicycling are also not explicitly considered, although they may be implicitly addressed by the inclusion of certain roadway facility type variables. Part of the reason why safety and security are not addressed in travel models is that research has yet to coalesce around the specific factors influencing perceptions of traffic safety and personal security.

To measure traffic safety, researchers have included proxy variables including traffic speeds and volumes, roadway facility types, traffic control devices, traffic calming installations, sidewalks, bicycle facilities, and other street design characteristics (Singleton \& Wang, 2014). Actual crash rates have rarely if ever been used to explain walking or bicycling travel behavior, likely in part because calculating exposure rates for these active modes is difficult without widespread and consistent pedestrian and bicyclist counts. In addition, perceptions of traffic safety may matter more than the rate at which rare collisions occur. For this reason, most public health researchers ask questions to ascertain perceptions of safety from traffic in one's neighborhood or area (Timperio et al., 2004).

To measure personal security, researchers have included certain types of land uses, alleyways, streetlights, and other measures such as graffiti, abandoned buildings, and trash (Boarnet et al., 2006). These urban form measures may not be significant in part because there are so many possible factors influencing security that any one may not contribute significantly. In contrast to traffic safety, actual crime rates have been used in 
some studies, with limited success (Ferrell et al., 2008; Hood et al., 2011; Singleton \& Wang, 2014). One reason for a lack of significant effects of crime is that crime variables are often spatially correlated with built environment measures, so it may be tough to capture their independent relationships (Ferrell et al., 2008). In addition, perceptions of personal security may matter more than the rate at which crimes occur. For this reason, most public health researchers also ask questions to ascertain perceptions of security from crime in one's neighborhood or area (Timperio et al., 2004).

Perceptions of safety and security, while more closely related to travel decisionmaking and thus travel behavior, might be tough to collect alongside travel survey diaries and may be difficult to manipulate. On the other hand, objectively-measured safety and security proxy variables are becoming more accessible, and interventions utilizing engineering and land use planning, while costly, may be more easily accommodated within existing local government capacities. Street design characteristics, while still difficult to obtain, have become increasingly available from state and municipal GIS asset databases. Crime rates and the location of "unsafe" or "undesirable" land uses are also more readily available today than in the past. A useful future study might try to relate perceived and objective measures of traffic safety and personal security, and then find associations between compound safety and security factors and walking and bicycling travel behavior.

\subsubsection{2.d. Cost}

The cost need is well documented in regional travel demand forecasting models in part because it has been a primary focus for so long. With recent interest in considering 
walking and bicycling, related cost data are not much more difficult to collect and represent. There are typically no monetary costs to walking or using a bicycle for personal transportation. Travel time (or distance) estimates for walking and cycling are captured even more simply than for auto and transit modes: using zone-based distance skims with assumed speeds (Singleton \& Clifton, 2013). Physical effort, mental effort, convenience, and reliability are not measured. Instead, travel time sometimes has a different coefficient for each mode to account for some of these other cost attributes (Cambridge Systematics et al., 2012).

Some improvements to walking and bicycling generalized cost data could allow operational travel models to better reflect aspects of the theory of travel decision-making. The use of more spatially-disaggregate analysis zones and complete street and sidewalk networks would allow for more realistic estimates of travel time by walking and cycling. Travel time could be segmented by other socioeconomic characteristics to allow different values of time. Although walking and cycling travel times tend to be relatively consistent, travel time (un)reliability for motorized modes could be included in travel models; data could come from traffic monitoring stations located on freeways, highways, and arterials, or from network routing simulations of congestion. Physical energy could be included using MET levels for various activities (Ainsworth et al., 2011), although it would likely be correlated with travel time. Mental effort might be difficult to measure.

\subsubsection{2.e. $\quad$ Pleasure}

Most environmental factors within the pleasure need have not been incorporated into travel demand forecasting models because they are difficult to measure (e.g., 
aesthetics) or incompatible with the operation of such models (e.g., weather). If travel models were to adopt different temporal or even seasonal interests, comfort could be included using expected weather; alternatively, weather could be an input to a method to seasonally-adjust travel demand estimates by mode. Similar to the cost need above, the exercise benefits of physical activity could be quantified using MET values for walking and bicycling (Ainsworth et al., 2011).

It may be inevitable that certain aspects of enjoyment - including socialization, travel liking, and particularly aesthetics - will be impossible to consistently measure and include in models due to heterogeneous values (Timms \& Tight, 2010). Others might disagree, pointing to evidence of strong consensus on aesthetic assessments among many demographic groups (Stamps, 1999). In addition, some researchers have begun to successfully operationalize such urban design qualities - imagability, enclosure, human scale, transparency, and complexity - with reliable and quantifiable factors (Ewing \& Handy, 2009). Travel liking might be included as an individual attitude that reduces the negative value of travel time in some instances.

In general, there might be little utility in gathering data on pleasure need factors because they may be less important than other needs and thus fail to show up as statistically significant in analyses. However, better means of controlling for other needs, including feasibility, accessibility, safety and security, and cost, might make it able for significant effects of walking and bicycling pleasure-related factors to emerge as significant. Similar to as was mentioned for the safety and security need, future work might try to relate environmental factors with perceptions of enjoyment, create a compound pleasure measure, and find associations with active travel behavior. 


\subsubsection{Moderation and Indirect Effects}

Demographic and socioeconomic data are some of the most commonly collected items on the household travel surveys used to inform and estimate regional travel demand models. Information on an individual's age, gender, race and ethnicity, worker status, student status, disability status, family relationships, household income, vehicle ownership, and housing characteristics are frequently reported (Federal Highway Administration, 2011). Such data could be readily used as factors influencing the feasibility of travel alternatives. A few limitations do occur: missing data or nonresponses are common for age and especially income (Stopher, 2012), and income categories may be less fine-grained that desired. A major barrier is the availability of data in large quantities: insufficient samples of travel survey records may preclude the estimation of a growing number of parameters necessary for testing many interactions with demographic and socioeconomic variables (Cohen et al., 2003; Snijders \& Bosker, 2012).

Methods for operationalizing the moderation hypothesis are also readily available. The most common way of implementing the moderation of travel choices by demographic and socioeconomic factors is through the inclusion of interaction terms. These interactions with key needs factors can be used with nominal (e.g., gender), ordinal (e.g., worker status), count (e.g., vehicle ownership), and continuous (e.g., age, income) demographic and socioeconomic variables. While most models may include such interactions only with time or cost, the theory suggests all factors within the hierarchy of travel needs may be moderated. Another way to operationalize the moderation hypothesis is through market segmentation: the use of separate travel models (or stages) for different 
types of travelers. One issue is how to classify people for segmentation: which demographic and socioeconomic factors to use, whether to segment a priori or use a statistical procedure such as cluster analysis, etc. Agent-based models can also accommodate moderation effects due to characteristics of the agent. Future work in this area could be helpful for operationalizing this relationship in travel models.

\subsubsection{Mediation}

Obtaining data to support the mediation hypothesis of perceptions for use in travel demand models appears challenging. There are many ways to measure beliefs, assessments, attitudes, norms, and other social-psychological or psychosocial influences on perceptions of travel needs fulfillment. Consistency, specificity, ease of understanding, and reliability are all important aspects of a desirable measure (Bohte et al., 2009) but may be challenging to achieve. A further difficulty is that socialpsychological travel factors are usually gathered in specialized studies and are rarely collected alongside the travel survey data used to estimate travel demand models (Stopher $\&$ Greaves, 2007). Even if these questions were included as part of a travel diary, the respondent burden may become excessive. Instead, psychosocial measures could be gathered for a subset of the sample or in a separate study. A benefit of a separate study focused solely on travel attitudes and perceptions is that it could be conducted more frequently than a regional household travel survey in order to capture fast-changing social and generational changes in how people prefer to get around.

Methods to analyze and incorporate the perception lens into travel demand models also require greater attention and work in the future. The hypothesis of mediated 
moderation states that the mediation should explain some of the moderation found; this in turn suggests that moderation would be empirically found without testing for any mediation effects. In the absence of data on travel attitudes and perceptions, good model fit might be obtained simply by including sufficient interactions with demographic and socioeconomic information. The addition of perceptive factors might improve a travel model's behavioral and policy sensitivity while not significantly improving its statistical goodness-of-fit.

A promising field of research is developing analytical methods to model these variations in attitudes and perceptions without directly asking questions about them. Many discrete choice modeling structures have been proposed and tested (in the literature, rarely in travel demand modeling practice) - including mixture models, mixed (or multilevel) logit, and latent class models - to accommodate what the economics field calls taste heterogeneity (Bhat, 2000; Fosgerau \& Hess, 2009; Greene \& Hensher, 2003; Hess, Bierlaire, \& Polak, 2007; Hess, Stathopoulos, Campbell, O’Neill, \& Caussade, 2013; Hess \& Train, 2011; Train, 2009; Wen, Wang, \& Fu, 2012). These methods might be able to represent the variation in travel attitudes and perceptions without direct survey questions, but require considerable skills and knowledge to understand and operate.

\subsubsection{Decision Rule}

The most widely-applied decision rule to model travel choices is utility maximization (or a probabilistic RUM derivation thereof). This is the basis of most discrete choice model structures used for destination and mode choice, including binary logit, multinomial logit, and nested logit regressions of utility. More advanced discrete 
choice models, including mixed or multilevel models and those of the generalized extreme value (GEV) class, also rely on RUM but can relax certain restrictive assumptions of simpler standard logit models (Koppelman \& Bhat, 2006; Train, 2009). Non-compensatory decision rules that do not assume utility maximization are also possible. These, including elimination by aspects (Tversky 1972a, 1972b) and attribute screening (Gilbride \& Allenby, 2005), could utilize RUM-based discrete choice model structures (Train, 2009). Latent class models might also accommodate heterogeneity among individuals or groups of people in the use of decision rules (Hess, Stathopolous, \& Daly, 2012).

While such advanced models have seen widespread development and interest among academics, they generally remain outside the workings of operational regional travel demand models. This could be due to their complexity, requiring both considerable skills and knowledge to interpret and often specialized software and extensive computational power to operate. In order to estimate parameters of interest, some model structures also require large datasets which may be beyond the financial capacities of many regional transportation planning organizations. Additionally, rarely are data gathered that could shed light on the decision rules used by travelers. The gap between theory and practice remains wide.

\subsubsection{Decision Contexts and Outcomes}

Most travel demand forecasting model systems assume a specific sequence of model operation, using a sequential series of models or a nested modeling structure in which lower choices are conditional upon higher-level choices. Rarely do travel models 
change the sequence in which these travel decisions occur, because such processes conform to travel behavior theory and have been proven successful in real-world implementations. Modeling multi-dimensional choices is possible, using some of the statistical methods mentioned in the Decision Rule section above. Some fruitful future work might test changing the sequence of decisions within travel demand models, at least for some purposes or situations.

\subsubsection{Feedback}

Data are a key limitation to the implementation of feedback mechanisms hypothesized by the theory of travel decision-making into regional travel demand models. Rarely is there information about an individual's past behavior, and even multi-day travel surveys in the US acquire less than a week's worth of travel diaries (Pendyala \& Pas, 2000), likely too little to make conclusions about habitual travel behavior. Some surveys (including the American Community Survey) ask respondents about typical travel modes and times-of-day for common journeys like to work and school, which can get at travel habits for these activities. However, travelers may not have one particular mode they use every day but instead may use different modes to work depending on other activities, weather, season, etc. Walking and bicycling commutes may be especially sensitive to these environmental conditions (Chen \& Clifton, 2012).

Another major challenge is how best to operationalize the concept of habitual travel behavior. Habit can be measured in a variety of ways, but the most common is a response-frequency measure using self-reported answers to questions about common travel destinations or modes (Verplanken et al., 1994). More recent work has developed 
alternative measures of habit to avoid some challenges with the older measure (Verplanken \& Orbell, 2003). While it may be possible to develop household travel survey questions to elicit responses necessary to construct a measure of habitual travel behavior, response burden would likely increase substantially, possibly increasing survey costs and degrading the quality of responses. Instead, these questions could be asked of a smaller subset of sampled respondents within the household travel survey, or they could be included in a separate more-frequent regional survey alongside questions of travel attitudes and perceptions.

Operationalizing the impact of new information, either through exploration of new choices or various types of interventions, on active travel behavior is even more challenging. Much more travel behavior research is needed before this concept could be incorporated into modeling practice. Once that happens, the influence of new information could conceptually be operationalized as follows. Perceptions of needs fulfillment (which modes are feasible, how safe is it to walk through this neighborhood, how long it would take to cycle) could be compared to objectively measurable factors of each need in the hierarchy. The amount of discord or difference between perceived and objective measures of each need (for each person or situation) could be a (random) model parameter. New information would then change this parameter (or its distribution) to bring perceptions more in line with reality.

\subsubsection{Summary}

To summarize, the state of data and analysis methods are sufficient to support some of the components and relationships within the theory of travel decision-making, 
while other aspects remain challenging. Data for the factors within the feasibility, accessibility, and cost needs, activity information, and demographic and socioeconomic characteristics are typically collected as part of household travel surveys, available through GIS databases, or becoming increasingly part of the datasets in the hands of regional transportation planning agencies. Data on the safety/security and pleasure needs, travel attitudes and preferences, and habitual travel behavior are rarely available or only captured in specialized studies that may not be compatible with travel demand modeling. Advances in environmental data collection and changes in spatial travel modeling structures prove promising to alleviate some of these data concerns for walking and bicycling.

Analysis methods to support the operationalization of the theory into regional travel demand models are generally in place, at least in academic literature. Some work is needed identifying the most relevant measures of accessibility, habit, and perceptions, as well as determining the decision rules individuals use to evaluate travel choices. There are promising methods to incorporate taste heterogeneity (or individual variation in perceptions of travel needs fulfillment) and non-compensatory decision rules into travel behavior analysis. However, these have seen limited implementation into operational transportation planning tools, in part because they often require extensive skills and data.

A final issue is related to applying such models in practice. Forecasting travel model inputs is a challenging process even for basic factors like travel cost (e.g., fuel and energy prices). If the theory of travel decision-making were to be fully operationalized in regional travel demand models, many new variables would need to be forecast well into the future. One reason why social-psychological factors have yet to see integration into 
travel forecasting tools is that transportation planners may feel that they are impossible to predict. One option might be to use travel demand models more as tools for scenario planning, testing the sensitivity of travel outcomes and performance measures to different future distributions of, for example, preferences for walking and bicycling. These uncertain inputs could also be used for policy sensitivity of non-engineering-based interventions. Forecasting model input data is a challenge, but it can also be an opportunity.

\subsection{Opportunities for Operationalization and Use in Travel Forecasting}

There are several short-term opportunities to apply parts of the theory of travel decision-making to improved travel demand models. One of the most straightforward ways to incorporate many suggestions from the theory is to transition, as many transportation planning agencies have done, from trip-based to activity-based models. ABMs generally do a better job than TBMs at representing the activity basis of travel demand, the feasibility need, and the multitude of travel (and travel-related) decision contexts and outcomes. Transitioning to an activity-based model system requires a considerable effort, but operating models exist to use as templates.

Incorporating more moderation factors is probably the lowest-hanging fruit for both TBMs and ABMs. Individual demographic and socioeconomic characteristics are already used or available for use in many model stages. Some segmentation of cost coefficients by income or auto ownership categories does occur in, for example, mode choice models; other factors like gender, age, and worker/student status are sometimes included as their own variables. More model estimations should test for significant 
interactions between presumed causal variables (time, cost, accessibility, and other factors from the hierarchy of travel needs) and demographic and socioeconomic characteristics, particularly for walk and bicycle mode choice utility functions. Of course, large sample sizes are required to achieve the power necessary to detect significant interaction terms (Cohen et al., 2003; Snijders \& Bosker, 2012). Testing for and including such interactions in travel models would make them more consistent with the behavioral theory expressed in this thesis as well as perhaps more sensitive to demographic shifts, in lieu of actual measurements of individual taste variation.

Other larger changes can improve how existing travel models reproduce the components and relationships in the theory without requiring additional research. Notably, utilizing smaller spatial scales and complete walking and cycling networks both for the calculation of key input variables (built environment factors, travel times) and for the operation of model procedures (trip generation, modal skims, route choice) might improve the accuracy of how travel models represent these active modes. Borrowing procedures from bicycle route choice studies and models, data could be collected and models estimated to assign walking and bicycling trips to the more detailed network, yielding more useful model outputs. Also, collecting data on and testing more built environment and urban/street design factors, especially those related to accessibility or representing traffic safety, might uncover significant relationships related to these two needs as well as increase travel models' sensitivities to land use and transportation interventions, projects, and scenarios.

More research would be necessary in order to address in models other remaining aspects of the theory. Travel behavior research needs to identify those factors - 
environmental, social, personal; policy-sensitive or not - that influence perceptions of safety, security, comfort, pleasure, and enjoyment while traveling. The relationships of attitudes, beliefs, perceptions, and habits on travel behavior, particularly surrounding walking and bicycling issues, needs more empirical support from longitudinal studies. More psychological research is also needed on compensatory and non-compensatory decision rules and processes related to making discrete travel choices. Another critical issue is the development of consistent and reliable measures all of these factors: safety/security, pleasure, perceptions, and habit. In order to be widely used and applied by modelers, who may be wary to include these "soft" aspects into travel models under their jurisdiction, these measures must be intuitive, relatively simple to calculate and interpret, and useful for the kinds of questions policy-makers may be asking. Making such changes to travel demand modeling practice requires significant investment in additional research, data collection, and modeling methods.

Hypothetically, there may be ways to conceive of such changes taking place in the future within the general structure of current travel demand forecasting models. Consider ways to integrate the perception lens. Just as households in TBMs are distributed among categories of household size, income, and life-cycle class, so could they be distributed among categories of attitudes towards travel modes. For example, individuals could be placed within one of the "four types of cyclists" (Geller, 2006; Dill \& McNeil, 2013), each of which has a different set of "perception parameters" which adjust the value of variables in the different aspects of the hierarchy of travel needs. The "no-way no-how" group would not consider bicycling to be a feasible alternative, while the "strong and fearless" would have a much lower value of safety and security when compared with the 
"interested but concerned" group. For the population synthesis process used in many ABMs, these perception parameters could be randomly drawn from a distribution of parameters observed in a dataset. For the forecast horizon year, distributions of parameters could be adjusted to reflect interventions being analyzed or societal trends anticipated.

If travel behavior research on environmental factors and psychological decision processes supports the classification of factors into the hierarchy of travel needs, models could be constructed to more directly match these categories. Many factors could combine to generate a single "feasibility factor", "accessibility index", or "safety and security score" that would then be used in model estimation to provide a single feasibility, accessibility, or safety/security parameter (or distribution of parameters). The process by which factors are combined into one value could be determined empirically (related to travel behavior outcomes), statistically (e.g., factor analysis), using modeler judgment, or even allowed to vary by individual characteristics. This process may be particularly useful for the accessibility need, where many measures of the built environment significant to active travel choices tend to be correlated. A factoring process would avoid some of the multicollinarity issues associated with estimating a model using several highly correlated variables.

Ignoring the necessity for travel behavior research to empirically validate such efforts, a primary need for these hypothetical adjustments to modeling practice is for data. For example, an initial major data collection effort on the scale of a regional household travel survey may be required in order to gather enough information on individual attitudes, beliefs, and preferences alongside observed or reported travel 
behavior. Research could then link these social-psychological factors to travel behavior in a manner applicable to travel demand model structures, perhaps as perception parameters that adjust the contribution of factors within the hierarchy of travel needs. Then, a much smaller data collection effort could be undertaken to annually or biennially take a representative sample of a region's "travel attitudes and values". The survey could be made relatively simple by removing the need to provide a detailed travel diary. Instead, the updated distribution of travel perceptions would be used to update the base model inputs without changing their relationship to travel outcomes. Such an effort would make models more flexible to respond to changing generational, cultural, and societal preferences about how people get around.

Making adjustments to travel demand modeling practice to bring it more in line with the theory of travel decision-making would yield many benefits. Travel models often receive much criticism from academic researchers, officials and policy-makers, and the general public for many reasons, including being: not based in travel behavior theory, insensitive to the transportation and land use changes under consideration, based in the past and unable to anticipate future generational and societal trends, poorly able to represent walking and bicycling, and simply a "black box". Such charges could be partially mitigated by some of the changes described above.

Relating the inner workings of a travel model to conceptual frameworks of travel behavior that are supported by empirical research could help dispel worries about the theoretical justification of travel models and perhaps open up the black box. Including many more environmental factors related to the hierarchy of travel needs would make models more sensitive to a wider array of infrastructure and development projects and 
policies. Being able to adjust model parameters for changes in travel attitudes and perceptions would at least improve upon travel models' abilities to more flexibly react to societal trends and changing preferences. All of these changes, including those of scale, would improve the models' abilities to represent walking and bicycling and the influences on these active travel modes.

Another significant benefit of this future generation of travel forecasting tools is that they could be able to be used to analyze many more types of interventions than are currently possible. For example, consider the many "E"s noted as necessary for efforts to improve traffic safety for vulnerable users and encourage walking and bicycling: engineering, education, encouragement, enforcement, and sometimes environment, emergency response, and evaluation and planning. These describe different approaches taken by various organizations that all seem to have an effect on changing levels of active travel. Yet, current travel demand models are able to predict changes as a result of only some engineering- and environment- (or land use-) based interventions.

Future travel forecasting models that incorporate aspects of the theory of travel decision-making may be sensitive to the array of "E" interventions, particularly education and encouragement. Educational efforts - including targeted informational campaigns (e.g., Portland's SmartTrips program) or training programs (like is done for cycling for most children in Germany, Denmark, and The Netherlands) - might have an effect on changing perceptions of modal awareness, availability, and self-efficacy for walking and bicycling. Research on the effects of these programs could show how they adjust a travel model's "perception parameters". Encouragement efforts - including commute challenges (e.g., local competitions, National Bike Challenge), walk/bike to work/school 
days, open streets events (e.g., Los Angeles' CicLAvia, New York City's Summer Streets, Portland's Sunday Parkways, San Francisco's Sunday Streets), and organized "fun" events and celebrations (e.g., Portland's Pedalpalooza and Walktober) - might also increase the awareness and pleasure of walking and bicycling. Given the right operationalization, these programs could have an effect on travel within a travel demand modeling system.

Another example application could be to incorporate the "four types of cyclists" into the travel forecasting tool. The current distribution of these types of people could be related to individual demographic and socioeconomic characteristics or used as a set of model parameters for perceptions of attainment of travel needs. Demographic shifts or interventions that are not captured by other model sensitivities (e.g., engineering-based infrastructure changes) could be forecast to change this set of parameters or this distribution of cyclist types, which would change resulting travel choices.

Alongside all of these modeling changes would be required an increased level of comfort on the part of modelers to use judgment about realistic future magnitudes of these "soft" interventions. Utilizing panels of experts or applying scenario testing could be other means of determining these newly-modelable impacts.

However, there may be concern about making travel demand models more complicated as informed by the theory of travel decision-making. There are diverging trends in the travel forecasting realm. On the one hand, travel demand models - as evidenced by $\mathrm{ABMs}$ and other advanced practices - are slowly becoming more behaviorally-representative by introducing more complex structures and additional data inputs, requiring longer model run times or more computational power. On the other 
hand, the development of more flexible transportation planning tools, particularly for scenario analysis, has received interest for their simpler structures, lower data requirements, and faster run times, allowing for rapid evaluation of multiple uncertain future scenarios. In either case, the existing travel model paradigm - as embodied by typical TBMs - is being seen as both structurally limiting and cumbersome.

Both kind of transportation planning tools may be needed in the future. It appears to be a major undertaking to incorporate all aspects of the theory of travel decisionmaking into a travel demand model that replicates observed behavior, let alone is able to forecast future travel. Perhaps the ideal use for such a fully-specified travel model is in evaluating the near-term $(<5$ years $)$ future and for project- and policy-specific forecasting. Long-term (> 20 years) travel forecasting may require a very different set of transportation planning and modeling tools, albeit ones that take inspiration from the theory of travel decision-making.

\subsection{Conclusion}

This chapter presented challenges and opportunities regarding one major contribution of the theory of travel decision-making: improving how travel demand forecasting models operate. First, a review of how walking and cycling are currently represented in travel demand models was presented. Next, the state of travel forecasting both trip- and activity-based models - was reviewed with respect to each component and relationships of the theory. To successfully operationalize the theory into modeling practice, three major aspects are necessary: research, data, and analysis methods. The state of travel behavior research was synthesized. Then, the availability of data and 
methods of analysis for each aspect of the theory were described. Finally, hypothetical opportunities for future integration and application of the theory to travel forecasting tools were discussed. These opportunities are of special interest to the author, and may be the focus of significant work in the future. 


\section{CONCLUSION: CONTRIBUTION}

My guess is that in fact for most travel demand applications, there is a RUM setup, perhaps enriched by some explicit structure to account for the formation and interaction of perceptions and attitudes, that will do a good job of representing behavior. This may not be a conventional MNL model with the usual measures of travel time and cost as explanatory variables, but I believe there is still a lot of room for travel demand analysts to develop richer and more realistic models of behavior within the paradigm of RUM, with consumers strongly motivated to maximize the desirability of perceived alternatives within a psychological context that may influence perceptions and tastes, and with modest extensions of traditional MNL functional forms. (McFadden, 2001, p. 38)

This thesis developed and discussed a unifying theory of travel decision-making with a focus on walking and bicycling and applications for travel demand modeling and forecasting. Chapter 2 provided a theoretical background by introducing a number of theories to explain (active) travel behavior from the fields of economics, geography, and psychology. Chapter 3 defined and described each component and relationship within the conceptual framework - activity, the hierarchy of travel needs, moderation, mediation, the decision rule, decision contexts and outcomes, and feedback - alongside supporting theoretical and empirical evidence. Chapter 4 discussed applications of the theory to travel demand modeling, including the state of travel forecasting, travel behavior 
research, data, and analysis methods with respect to each aspect of the theory. The thesis closed with an analysis of future opportunities.

This work has made several major contributions. First, the theory of travel decision-making provides a unifying framework of travel behavior to inform academic research and future theories. The general academic realm dealing with travel behavior including the fields of microeconomics, transport geography, social/environmental psychology, transportation engineering, and urban studies and planning - has yet to settle on a comprehensive theory of travel behavior that accounts for the complex relationships between (and among) personal, social, and environmental factors. Each field often approaches the issue of analyzing travel behavior from their own point of view, utilizing a separate canon. A few scholars have attempted to link these travel behavior explanations together, some with a focus on walking and bicycling, including the theory of routine mode choice decisions (Schneider, 2013) and the hierarchy of walking needs (Alfonzo, 2005).

The theory of travel decision-making builds off of these efforts to craft a unifying interdisciplinary framework of travel behavior that incorporates insights from the fields of economics, geography, and psychology. Notably, the theory posits a hierarchy of travel needs to organize significant individual and environmental factors and upon which to evaluate travel options. It importantly suggests that individual demographic and socioeconomic factors only indirectly affect the travel decision-making process, contrary to the way in which such factors are currently treated in research and practice. The concept of the perception lens as a way of explaining how objectively-measured factors vary in their interpretation and influence is a critical insight for making the leap between 
a conceptual theory and an analytically-testable hypothesis. This theoretical framework can provide guidance to travel behavior researchers as they design studies, analyze data, construct theories, and inform transportation planning and policy-making.

A second major contribution is that the theory is designed to be directly applicable to the practice of travel demand modeling and forecasting. Travel demand forecasting models are among the most institutionalized transportation planning tools used by local decision-makers to forecast future travel needs and make transportation policy and investment decisions. However, despite recent efforts, most models lack a strong derivation from travel behavior theory and often operate without due consideration of a human decision-making process. In addition, these tools are increasingly being asked to address additional policy concerns, including aspects of multimodal performance measurement, land use development, air quality, emissions, climate change, public health, and non-motorized modes.

The theory of travel decision-making and its components and relationships are directly applicable to how travel demand models could be structured because it was developed with modeling applications particularly in mind. It attempts to forge a way forward for the successful integration of economic and psychological theories of travel behavior into operational transportation planning tools (McFadden, 2001). Although the thesis notes current limitations in areas of modeling practice, travel behavior research, data, and analysis methods, it also begins to suggest some ways in which aspects of the theory can be operationalized and integrated into travel forecasting tools. Of particular utility in the short run is the testing of interactions of factors with individual characteristics beyond income. Travel models might become much more useful in the 
long run by collecting and including social-psychological factors relating to the perception lens. Such adjustments might make travel demand models able to analyze many more types of interventions beyond engineering and land use policies, including less-tangible education and encouragement efforts.

The final significant contribution of this thesis is towards the study of and planning for active modes of travel. Although increasing walking and bicycling is a policy interest in many US urban areas, the transportation planning tools used in such processes, particularly travel demand models, often inadequately represent these active travel modes or are insensitive to their influences. Among the reasons for this state are data limitations, complex or inflexible methods of analysis, and the lack of a comprehensive theory of travel behavior that accounts for the unique and complex factors influencing active travel decisions.

Although it is more generally applicable to all modes and means of personal transportation, the theory of travel decision-making was explicitly designed to account for factors and considerations of importance to potential active travelers. Although not a panacea, it does partially answer calls for better conceptual models to guide future studies of the effects on active travel (Owen et al., 2004; Handy, 2005; Saelens \& Handy, 2008). In addition, it suggests one way to conceptualize active travel behavior that is directly applicable to travel demand modeling. A unifying framework - such as the theory of travel decision-making presented in this thesis - including walking and bicycling behavioral relationships from multiple disciplines yet structured with travel forecasting applications in mind could make significant contributions to active travel research, planning, and practice. 
This thesis and the theory developed within it are not without their limitations. The theory's focus on short-term, day-to-day decision-making considers longer-term vehicle ownership, residential location, and lifestyle decisions (Van Acker et al., 2010) to be exogenous inputs. The extent to which short-term travel decisions influence these decisions is outside the scope of the theory. On a minor note, the theory also does not attempt to address activity decisions and assumes a degree of autonomy over one's travel decisions. A more important limitation is based on its theoretical nature: the components and relationships expressed in the theory of travel decision-making have yet to be empirically tested and repeatedly verified, either as specified or as a collective group.

Furthermore, the theory is not yet ready to be fully applied to the design of improved travel demand models. Although some aspects of the theory are already well integrated into practice, a lack of knowledge from travel behavior research and difficulties gathering data and measuring items clouds the applicability and operationalization of some components. The field has yet to identify the environmental factors that lead to perceptions of safety and security as well as the pleasure of certain travel options, especially while walking and cycling. In particular, researchers need better ways to simply measure and collect information on social-psychological factors that make up the perception lens and knowledge on the relations of perceptions and habits to travel behavior. Research should also investigate non-compensatory decision rules. Much more work is needed confirming aspects of the theory and creating means to operationalize them into travel demand models.

Future work will start taking aim at some of these limitations. In general, each of the hypothesized relationships between components of the theory should be tested using 
empirical travel behavior data, preferably from related datasets. Of particular interest are developing ways to measure and forecast perceptive aspects as well as relate them backwards to the hierarchy of travel needs and forwards to travel behavior. Investigating the structure and composition of the hierarchy, especially determining its relative order and relevant factors, would be fruitful. At the same time, it would be intriguing and valuable - particularly in the forecasting of walking and bicycling - to begin working on ways of operationalizing the relationships of the theory into travel demand models. A proof-of-concept model system could even be developed using synthetic data to demonstrate the feasibility and usefulness of such a transportation planning tool.

This thesis, and the theory of travel decision-making developed herein, fills a major gap in travel behavior knowledge and literature and has significant contributions to travel demand forecasting and the study and planning of walking and bicycling. Of course, the conceptual framework is not infallible. Other researchers may come to different conclusions about the state of empirical evidence from travel behavior research or hypothesize different ways to structure and relate influences on walking and bicycling. In addition, others may find more successful ways to include aspects excluded from the focus of this theory. However, this thesis does present one way to conceptualize influences on walk- and bicycle-related travel decisions, with concern over the theory's ability to inform travel behavior research and have applications to travel forecasting. It offers a way to improve both our knowledge and our transportation planning processes' representation of walking and bicycling. The author looks forward to working over the coming years on refining, proving, or disproving aspects of the theory, as well as proposing ways to operationalize and apply it to travel demand forecasting models. 


\section{REFERENCES}

Aarts, H., \& Dijksterhuis, A. (2000). The automatic activation of goal-directed behaviour: The case of travel habit. Journal of Environmental Psychology, 20(1), 75-82. http://dx.doi.org/10.1006/jevp.1999.0156

Aarts, H., Verplanken, B., \& van Knippenberg, A. (1997). Habit and information use in travel mode choices. Acta Psychologica, 96(1), 1-14. http://dx.doi.org/10.1016/S0001-6918(97)00008-5

Aarts, H., Verplanken, B., \& van Knippenberg, A. (1998). Predicting behavior from actions in the past: Repeated decision making or a matter of habit? Journal of Applied Social Psychology, 28(15), 1355-1374. http://dx.doi.org/10.1111/j.15591816.1998.tb01681.x

Abraham, J. E., \& Hunt, J. D. (1997). Specification and estimation of nested logit model of home, workplaces, and commuter mode choices by multiple-worker households. Transportation Research Record: Journal of the Transportation Research Board, 1606, 17-24. http://dx.doi.org/10.3141/1606-03

Adams, J., \& White, M. (2003). Are activity promotion interventions based on the transtheoretical model effective? A critical review. British Journal of Sports Medicine, 37(2), 106-114. http://dx.doi.org/10.1136/bjsm.37.2.106

Adkins, A., Dill, J., Luhr, G., \& Neal, M. (2012). Unpacking walkability: Testing the influence of urban design features on perceptions of walking environment attractiveness. Journal of Urban Design, 17(4), 499-510. http://dx.doi.org/10.1080/13574809.2012.706365 
Adler, T. J., \& Ben-Akiva, M. (1976). Joint-choice model for frequency, destination, and travel mode for shopping trips. Transportation Research Record, 569, 136-150.

Agrawal, A. W., Schlossberg, M., \& Irvin, K. (2008). How far, by which route, and why? A spatial analysis of pedestrian preference. Journal of Urban Design, 13(1), 8198. http://dx.doi.org/10.1080/13574800701804074

Ainsworth, B. E., Haskell, W. L., Herrmann, S. D., Meckes, N., Bassett Jr., D. R., Tudor-Locke, C., ... Leon, A. S. (2011). The compendium of physical activities tracking guide. Healthy Lifestyles Research Center, College of Nursing \& Health Innovation, Arizona State University. Retrieved from https://sites.google.com/site/compendiumofphysicalactivities/

Ajzen, I. (1991). The theory of planned behavior. Organizational Behavior and Human Decision Processes, 50(2), 179-211. http://dx.doi.org/10.1016/07495978(91)90020-T

Akar, G. (2009). Analysis of activity choice: The role of activity attributes and individual schedules. (Unpublished doctoral dissertation). University of Maryland, College Park, MD. Retrieved from http://hdl.handle.net/1903/9594

Alfonzo, M. A. (2005). To walk or not to walk? The hierarchy of walking needs. Environment and Behavior, 37(6), 808-836. http://dx.doi.org/10.1177/0013916504274016

Algers, S., Eliasson, J., \& Mattsson, L. G. (2005). Is it time to use activity-based urban transport models? A discussion of planning needs and modelling possibilities. The Annals of Regional Science, 39(4), 767-789. http://dx.doi.org/10.1007/s00168005-0016-8 
Anable, J., \& Gatersleben, B. (2005). All work and no play? The role of instrumental and affective factors in work and leisure journeys by different travel modes. Transportation Research Part A: Policy and Practice, 39(2), 163-181. http://dx.doi.org/10.1016/j.tra.2004.09.008

Appleyard, B. (2012). Sustainable and healthy travel choices and the built environment. Transportation Research Record: Journal of the Transportation Research Board, 2303, 38-45. http://dx.doi.org/10.3141/2303-05

Arentze, T., Hofman, F., van Mourik, H., \& Timmermans, H. (2000). ALBATROSS: Multiagent, rule-based model of activity pattern decisions. Transportation Research Record: Journal of the Transportation Research Board, 1706, 136-144. http://dx.doi.org/10.3141/1706-16

Axhausen, K. W., \& Gärling, T. (1992). Activity-based approaches to travel analysis: Conceptual frameworks, models, and research problems. Transport Reviews, 12(4), 323-341. http://dx.doi.org/10.1080/01441649208716826

Badland, H., \& Schofield, G. (2005). Transport, urban design, and physical activity: An evidence-based update. Transportation Research Part D: Transport and Environment, 10(3), 177-196. http://dx.doi.org/10.1016/j.trd.2004.12.001

Badoe, D. A., \& Miller, E. J. (2000). Transportation-land-use interaction: Empirical findings in North America, and their implications for modeling. Transportation Research Part D: Transport and Environment, 5(4), 235-263. http://dx.doi.org/10.1016/S1361-9209(99)00036-X 
Bagley, M. N., \& Mokhtarian, P. L. (2002). The impact of residential neighborhood type on travel behavior: A structural equations modeling approach. The Annals of Regional Science, 36(2), 279-297. http://dx.doi.org/10.1007/s001680200083

Ball, K., Bauman, A., Leslie, E., \& Owen, N. (2001). Perceived environmental aesthetics and convenience and company are associated with walking for exercise among Australian adults. Preventive Medicine, 33(5), 434-440. http://dx.doi.org/10.1006/pmed.2001.0912

Ball, K., Crawford, D., \& Owen, N. (2000). Obesity as a barrier to physical activity. Australian and New Zealand Journal of Public Health, 24(3), 331-333. http://dx.doi.org/10.1111/j.1467-842X.2000.tb01579.x

Bamberg, S. (2006). Is a residential relocation a good opportunity to change people's travel behavior? Results from a theory-driven intervention study. Environment and Behavior, 38(6), 820-840. http://dx.doi.org/10.1177/0013916505285091

Bamberg, S. (2007). Is a stage model a useful approach to explain car drivers' willingness to use public transportation? Journal of Applied Social Psychology, 37(8), 17571783. http://dx.doi.org/10.1111/j.1559-1816.2007.00236.x

Bamberg, S., Ajzen, I., \& Schmidt, P. (2003). Choice of travel mode in the theory of planned behavior: The roles of past behavior, habit, and reasoned action. Basic and Applied Social Psychology, 25(3), 175-187. http://dx.doi.org/10.1207/S15324834BASP2503_01

Bamberg, S., Hunecke, M., \& Blöbaum, A. (2007). Social context, personal norms and the use of public transportation: Two field studies. Journal of Environmental Psychology, 27(3), 190-203. http://dx.doi.org/10.1016/j.jenvp.2007.04.001 
Bamberg, S., Rölle, D., \& Weber, C. (2003). Does habitual car use not lead to more resistance to change of travel mode?. Transportation, 30(1), 97-108. http://dx.doi.org/10.1023/A:1021282523910

Bamberg, S., \& Schmidt, P. (1998). Changing travel-mode choice as rational choice: Results from a longitudinal intervention study. Rationality and Society, 10(2), 223-252. http://dx.doi.org/10.1177/104346398010002005

Bamberg, S., \& Schmidt, P. (2003). Incentives, morality, or habit? Predicting students' car use for university routes with the models of Ajzen, Schwartz, and Triandis. Environment and Behavior, 35(2), 264-285. http://dx.doi.org/10.1177/0013916502250134

Bandura, A. (1977). Self-efficacy: Toward a unifying theory of behavioral change. Psychological Review, 84(2), 191-215. http://dx.doi.org/10.1037/0033295X.84.2.191

Bandura, A. (1986). Social foundations of thought and action: A social cognitive theory. Englewood Cliffs, NJ: Prentice-Hall, Inc.

Baran, P. K., Rodríguez, D. A., \& Khattak, A. J. (2008). Space syntax and walking in a new urbanist and suburban neighbourhoods. Journal of Urban Design, 13(1), 528. http://dx.doi.org/10.1080/13574800701803498

Bates, J., Polak, J., Jones, P., \& Cook, A. (2001). The valuation of reliability for personal travel. Transportation Research Part E: Logistics and Transportation Review, 37(2), 191-229. http://dx.doi.org/10.1016/S1366-5545(00)00011-9 
Ben-Akiva, M., Bergman, M. J., Daly, A. J., \& Ramaswamy, R. (1984). Modeling interurban route choice behaviour. Proceedings of the Ninth International Symposium on Transportation and Traffic Theory, 299-300.

Ben-Akiva, M. E., \& Bowman, J. L. (1998a). Activity based travel demand model systems. In P. Marcotte \& S. Nguyen (Eds.), Equilibrium and advanced transportation modeling (pp. 27-46). Boston, MA: Kluwer Academic Publishers.

Ben-Akiva, M., \& Bowman, J. L. (1998b). Integration of an activity-based model system and a residential location model. Urban Studies, 35(7), 1131-1153. http://dx.doi.org/10.1080/0042098984529

Ben-Akiva, M., \& Lerman, S. R. (1985). Discrete choice analysis: Theory and application to travel demand. Cambridge, MA: The MIT Press.

Ben-Akiva, M. E., Ramming, M. S., \& Walker, J. L. (1999). Improving behavioral realism of urban transportation models through explicit treatment of individual's spatial ability. Presented at the European Science Foundation/US National Science Foundation Conference on Social Change and Sustainable Transport, Berkeley, CA.

Berrigan, D., Pickle, L. W., \& Dill, J. (2010). Associations between street connectivity and active transportation. International Journal of Health Geographics, 9(1), 20. http://dx.doi.org/10.1186/1476-072X-9-20

Berrigan, D., \& Troiano, R. P. (2002). The association between urban form and physical activity in US adults. American Journal of Preventive Medicine, 23(2), 74-79. http://dx.doi.org/10.1016/S0749-3797(02)00476-2 
Bhat, C. R. (1997). Work travel mode choice and number of non-work commute stops. Transportation Research Part B: Methodological, 31(1), 41-54. http://dx.doi.org/10.1016/S0191-2615(96)00016-1

Bhat, C. R. (2000). Incorporating observed and unobserved heterogeneity in urban work travel mode choice modeling. Transportation Science, 34(2), 228-238. http://dx.doi.org/10.1287/trsc.34.2.228.12306

Bhat, C. R., \& Guo, J. Y. (2007). A comprehensive analysis of built environment characteristics on household residential choice and auto ownership levels. Transportation Research Part B: Methodological, 41(5), 506-526. http://dx.doi.org/10.1016/j.trb.2005.12.005

Bhat, C. R., \& Sardesai, R. (2006). The impact of stop-making and travel time reliability on commute mode choice. Transportation Research Part B: Methodological, 40(9), 709-730. http://dx.doi.org/10.1016/j.trb.2005.09.008

Black, C., Collins, A., \& Snell, M. (2001). Encouraging walking: the case of journey-toschool trips in compact urban areas. Urban Studies, 38(7), 1121-1141. http://dx.doi.org/10.1080/00420980124102

Block, H. D., \& Marschak, J. (1960). Random orderings and stochastic theories of responses. Contributions to Probability and Statistics, 2, 97-132.

Boarnet, M. G., Anderson, C. L., Day, K., McMillan, T., \& Alfonzo, M. (2005). Evaluation of the California Safe Routes to School legislation: Urban form changes and children's active transportation to school. American Journal of Preventive $\quad$ Medicine, $\quad 28(2), \quad 134-140$. http://dx.doi.org/10.1016/j.amepre.2004.10.026 
Boarnet, M. G., Day, K., Alfonzo, M., Forsyth, A., \& Oakes, M. (2006). The IrvineMinnesota inventory to measure built environments: Reliability tests. American Journal of Preventive Medicine, 30(2), 153-159. http://dx.doi.org/10.1016/j.amepre.2005.09.018

Boarnet, M. G., Forsyth, A., Day, K., \& Oakes, J. M. (2011). The street level built environment and physical activity and walking: Results of a predictive validity study for the Irvine Minnesota inventory. Environment and Behavior, 43(6), 735775. http://dx.doi.org/10.1177/0013916510379760

Bohte, W., Maat, K., \& van Wee, B. (2009). Measuring attitudes in research on residential self-selection and travel behaviour: A review of theories and empirical $\begin{array}{llll}\text { research. } & \text { Transport } & \text { Reviews, } & \text { 29(3), }\end{array}$ http://dx.doi.org/10.1080/01441640902808441

Bomberg, M., Zorn, L., \& Sall, E. (2013). Incorporating user based perspective of livability projects in SF-CHAMP mode choice models. Transportation Letters: the International Journal of Transportation Research, 5(2), 83-95. http://dx.doi.org/10.1179/1942786713Z.00000000010

Booth, M. L., Owen, N., Bauman, A., Clavisi, O., \& Leslie, E. (2000). Social-cognitive and perceived environment influences associated with physical activity in older Australians. Preventive $\quad$ Medicine, $31(1), \quad$ 15-22. http://dx.doi.org/10.1006/pmed.2000.0661

Borgers, E. A., Viti, F., \& Hoogendoorn, S. P. (2005). Joint modeling of advanced travel information service, habit, and learning impacts on route choice by laboratory simulator experiments. Transportation Research Record: Journal of the 
Transportation Research Board, 1926, 189-197. http://dx.doi.org/10.3141/192622

Bovy, P. H. (2009). On modelling route choice sets in transportation networks: A $\begin{array}{llll}\text { synthesis. } & \text { Transport } & \text { Reviews, } & \text { 29(1), }\end{array}$ http://dx.doi.org/10.1080/01441640802078673

Bovy, P. H., \& Fiorenzo-Catalano, S. (2007). Stochastic route choice set generation: Behavioral and probabilistic foundations. Transportmetrica, 3(3), 173-189. http://dx.doi.org/10.1080/18128600708685672

Bowman, J. L., \& Ben-Akiva, M. (1997). Activity based travel forecasting. Presented at the Activity Based Travel Forecasting Conference, New Orleans, LA.

Bowman, J. L., \& Ben-Akiva, M. E. (2001). Activity-based disaggregate travel demand model system with activity schedules. Transportation Research Part A: Policy and Practice, 35(1), 1-28. http://dx.doi.org/10.1016/S0965-8564(99)00043-9

Bradley, M., \& Vovsha, P. (2005). A model for joint choice of daily activity pattern types of household members. Transportation, 32(5), 545-571. http://dx.doi.org/10.1007/s11116-005-5761-0

Brennand, A. W. (2011). Incorporating travel time reliability in the estimation of assignment models (Research Report 464). Wellington, NZ: NZ Transport Agency. Retrieved from http://www.nzta.govt.nz/resources/research/reports/464/

Broach, J., Dill, J., \& Gliebe, J. (2012). Where do cyclists ride? A route choice model developed with revealed preference GPS data. Transportation Research Part A: Policy and Practice, 46, 1730-1740. http://dx.doi.org/10.1016/j.tra.2012.07.005 
Broach, J., \& Dill, J. (2013). Modeling children's independent walk and bike travel to parks and school. Presented at the 92nd Annual Meeting of the Transportation Research Board, Washington, DC.

Broach, J., Gliebe, J., \& Dill, J. (2010). Calibrated labeling method for generating bicyclist route choice sets incorporating unbiased attribute variation. Transportation Research Record: Journal of the Transportation Research Board, 2197, 89-97. http://dx.doi.org/DOI: 10.3141/2197-11

Broach, J., Gliebe, J., \& Dill, J. (2011). Bicycle route choice model developed using revealed preference GPS data. Presented at the 90th Annual Meeting of the Transportation Research Board, Washington, DC.

Brownson, R. C., Baker, E. A., Housemann, R. A., Brennan, L. K., \& Bacak, S. J. (2001). Environmental and policy determinants of physical activity in the United States. American Journal of Public Health, 91(12), 1995-2003. http://dx.doi.org/10.2105/AJPH.91.12.1995

Brownstone, D., \& Small, K. A. (2005). Valuing time and reliability: assessing the evidence from road pricing demonstrations. Transportation Research Part A: Policy and Practice, 39(4), 279-293. http://dx.doi.org/10.1016/j.tra.2004.11.001

Cambridge Systematics, Inc., \& Barton Aschman Associates. (1994). Short-term travel model improvements: Final report (Publication No. DOT-T-95-05). Washington, DC: U.S. Department of Transportation. Retrieved from http://ntl.bts.gov/DOCS/445.html

Cambridge Systematics, Inc., \& Parsons, Brinckerhoff, Quade and Douglas. (1996). Making the land use transportation air quality connection: Analysis of 
alternatives: Volume 5. Portland, OR: 1000 Friends of Oregon. Retrieved from http://www.friends.org/resources/reports

Cambridge Systematics, Inc., Parsons, Brinckerhoff, Quade and Douglas, \& S. H. Putman Associates, Inc. (1996). Making the land use transportation air quality connection: Model modifications: Volume 4. Portland, OR: 1000 Friends of Oregon. Retrieved from http://www.friends.org/resources/reports

Cambridge Systematics, Inc., Vanasse Hangen Brustlin, Inc., Gallop Corporation, Bhat, C. R., Shapiro Transportation Consulting, LLC, \& Martin/Alexiou/Bryson, PLLC. (2012). Travel demand forecasting: Parameters and techniques (NCHRP Report 716). Washington, DC: Transportation Research Board. Retrieved from http://onlinepubs.trb.org/onlinepubs/nchrp/nchrp_rpt_716.pdf

Cao, X., Handy, S. L., \& Mokhtarian, P. L. (2006). The influences of the built environment and residential self-selection on pedestrian behavior: Evidence from Austin, TX. Transportation, 33(1), 1-20. http://dx.doi.org/10.1007/s11116-005$7027-2$

Cao, X., Mokhtarian, P. L., \& Handy, S. L. (2007). Cross-sectional and quasi-panel explorations of the connection between the built environment and auto ownership. Environment and Planning A, 39, 830-847. http://dx.doi.org/10.1068/a37437

Cao, X., Mokhtarian, P. L., \& Handy, S. L. (2009). Examining the impacts of residential self-selection on travel behaviour: A focus on empirical findings. Transport Reviews, 29(3), 359-395. http://dx.doi.org/10.1080/01441640802539195

Cao, X., Mokhtarian, P. L., \& Handy, S. L. (2010). Neighborhood design and the accessibility of the elderly: An empirical analysis in Northern California. 
International Journal of Sustainable Transportation, 4(6), 347-371. http://dx.doi.org/10.1080/15568310903145212

Carrion, C., \& Levinson, D. (2012). Value of travel time reliability: A review of current evidence. Transportation Research Part A: Policy and Practice, 46(4), 720-741. http://dx.doi.org/10.1016/j.tra.2012.01.003

Cervero, R., \& Kockelman, K. (1997). Travel demand and the 3Ds: Density, diversity, and design. Transportation Research Part D: Transport and Environment, 2(3), 199-219. http://dx.doi.org/10.1016/S1361-9209(97)00009-6

Cervero, R., \& Duncan, M. (2003). Walking, bicycling, and urban landscapes: Evidence from the San Francisco Bay Area. American Journal of Public Health, 93(9), 1478-1483. http://dx.doi.org/10.2105/AJPH.93.9.1478

Chapin Jr., F. S. (1974). Human activity patterns in the city: Things people do in time and in space. New York, NY: John Wiley \& Sons, Inc.

Chen, R. B., \& Clifton, K. J. (2012). Traveling in comfort: Investigating weather ranges for travel. Presented at the $91^{\text {st }}$ Annual Meeting of the Transportation Research Board, Washington, DC.

Clifton, K. J., \& Dill, J. (2005). Women's travel behavior and land use: Will new styles of neighborhoods lead to more women walking? Research on Women's Issues in Transportation: Report of a Conference, 2, 89-99. Retrieved from http://onlinepubs.trb.org/onlinepubs/conf/CP35v2.pdf

Clifton, K., Ewing, R., Knaap, G. J., \& Song, Y. (2008). Quantitative analysis of urban form: A multidisciplinary review. Journal of Urbanism, 1(1), 17-45. http://dx.doi.org/10.1080/17549170801903496 
Clifton, K., J., \& Livi, A. D. (2005). Gender differences in walking behavior, attitudes about walking, and perceptions of the environment in three Maryland communities. Research on Women's Issues in Transportation: Report of a $\begin{array}{lllll}\text { Conference, } & 2, & \text { 79-88. } & \text { Retrieved }\end{array}$ http://onlinepubs.trb.org/onlinepubs/conf/CP35v2.pdf

Clifton, K. J., \& Muhs, C. D. (2012). Capturing and representing multimodal trips in travel surveys: Review of the practice. Transportation Research Record: Journal of the Transportation Research Board, 2285, 74-83. http://dx.doi.org/10.3141/2285-09

Cohen, J., Cohen, P., West, S. G., \& Aiken, L. S. (2003). Applied multiple regression/correlation analysis for the behavioral sciences $\left(3^{\text {rd }}\right.$ ed.). New York, NY: Routledge.

Collantes, G. O., \& Mokhtarian, P. L. (2007). Subjective assessments of personal mobility: What makes the difference between a little and a lot? Transport Policy, 14(3), 181-192. http://dx.doi.org/10.1016/j.tranpol.2006.12.002

Committee for Determination of the State of the Practice in Metropolitan Area Travel Forecasting. (2007). Metropolitan travel forecasting: Current practice and future direction (Special Report 288). Washington, DC: Transportation Research Board. Retrieved from http://onlinepubs.trb.org/onlinepubs/sr/sr288.pdf

Cooper, C. (2007). Successfully changing individual travel behavior: Applying community-based social marketing to travel choice. Transportation Research Record: Journal of the Transportation Research Board, 2021, 89-99. http://dx.doi.org/10.3141/2021-11 
Crane, R. (2000). The influence of urban form on travel: An interpretive review. Journal of Planning $\quad$ Literature, $\quad$ 15(1), http://dx.doi.org/10.1177/08854120022092890

Crews, J. E, \& Campbell, V. A. (2001). Health conditions, activity limitations, and participation restrictions among older people with visual impairments. Journal of Visual Impairment \& Blindness, 95(8), 453-467. Retrieved from http://www.afb.org/jvib/jvibabstractNew.asp?articleid=JVIB950802

Cunningham, G., \& Michael, Y. L. (2004). Concepts guiding the study of the impact of the built environment on physical activity for older adults: A review of the literature. American Journal of Health Promotion, 18(6), 435-443. http://dx.doi.org/10.4278/0890-1171-18.6.435

Day, K., Boarnet, M., Alfonzo, M., \& Forsyth, A. (2006). The Irvine-Minnesota inventory to measure built environments: Development. American Journal of Preventive $\quad$ Medicine, $\quad 30(2), \quad$ 144-152. http://dx.doi.org/10.1016/j.amepre.2005.09.017

De Bourdeaudhuij, I., Sallis, J. F., \& Saelens, B. E. (2003). Environmental correlates of physical activity in a sample of Belgian adults. American Journal of Health Promotion, 18(1), 83-92. http://dx.doi.org/10.4278/0890-1171-18.1.83

De Bourdeaudhuij, I., Teixeira, P. J., Cardon, G., \& Deforche, B. (2005). Environmental and psychosocial correlates of physical activity in Portuguese and Belgian adults. Public Health Nutrition, 8(7), 886-895. http://dx.doi.org/10.1079/PHN2005735 
Dieleman, F. M., Dijst, M., \& Burghouwt, G. (2002). Urban form and travel behavior: Micro-level household attributes and residential context. Urban Studies, 39(3), 507-527. http://dx.doi.org/10.1080/00420980220112801

Dill, J. (2004). Measuring network connectivity for bicycling and walking. Presented at the 83rd Annual Meeting of the Transportation Research Board, Washington, DC.

Dill, J., \& McNeil, N. (2013). Four types of cyclists? Examining a topology to better understand bicycling behavior and potential. Presented at the 92nd Annual Meeting of the Transportation Research Board, Washington, DC.

Doherty, S. T., Miller, E. J., Axhausen, K. W., \& Gärling, T. (2001). A conceptual model of the weekly household activity-travel scheduling process. In E. Stern, I. Saloman, \& P. Bovy (Eds.), Travel behavior: Patterns, implications and modeling (pp. 148-165). Cheltenham, UK: Elgar Publishing Ltd.

Domarchi, C., Tudela, A., \& González, A. (2008). Effect of attitudes, habit and affective appraisal on mode choice: An application to university workers. Transportation, 35(5), 585-599. http://dx.doi.org/10.1007/s11116-008-9168-6

Domencich, T. A., \& McFadden, D. (1975). Urban travel demand: A behavioral analysis. Amsterdam, The Netherlands: North-Holland Publishing Company.

Donnelly, R., Erhardt, G. D., Moeckel, R., \& Davidson, W. A. (2010). Advanced practices in travel forecasting: A synthesis of highway practice (NCHRP Synthesis 406). Washington, DC: Transportation Research Board. Retrieved from http://onlinepubs.trb.org/onlinepubs/nchrp/nchrp_syn_406.pdf 
Dupuit, J. (1952). On the measurement of the utility of public works. International Economic Papers, 2. (R. H. Barback, Trans.). London, UK: Macmillan Co. (Original work published 1844 in Annals des ponts et chaussees, 2).

Dziekan, K. (2008). What do people know about their public transport options? Transportation, 35(4), 519-538. http://dx.doi.org/10.1007/s11116-008-9164-x

Eash, R. (1997). Incorporating urban design variables in metropolitan planning organizations' travel demand models. Urban design, telecommuting and travel forecasting conference: Summary, recommendations, and compendium of papers (Publication No. DOT-T-98-2) Arlington, TX: Texas Transportation Institute. Retrieved from http://media.tmiponline.org/clearinghouse/udes/eash.htm

Eash, R. (1999). Destination and mode choice models for nonmotorized travel. Transportation Research Record: Journal of the Transportation Research Board, 1674, 1-8. http://dx.doi.org/10.3141/1674-01

Eriksson, L., Garvill, J., \& Nordlund, A. M. (2008). Interrupting habitual car use: The importance of car habit strength and moral motivation for personal car use reduction. Transportation Research Part F: Traffic Psychology and Behaviour, 11(1), 10-23. http://dx.doi.org/10.1016/j.trf.2007.05.004

Ettema, D. \& Timmermans, H. (1997). Theories and models of activity patterns. In D. Ettema \& H. Timmermans (Eds.), Activity-based approaches to travel analysis (pp. 1-36). Oxford, UK: Pergamon.

Ewing, R., \& Cervero, R. (2001). Travel and the built environment: A synthesis. Transportation Research Record: Journal of the Transportation Research Board, 1780, 87-114. http://dx.doi.org/10.3141/1780-10 
Ewing, R., \& Cervero, R. (2010). Travel and the built environment: A meta-analysis. Journal of the American Planning Association, 76(3), 265-294. http://dx.doi.org/10.1080/01944361003766766

Ewing, R., \& Dumbaugh, E. (2009). The built environment and traffic safety: A review of empirical evidence. Journal of Planning Literature, 23(4), 347-367. http://dx.doi.org/10.1177/0885412209335553

Ewing, R., \& Handy, S. (2009). Measuring the unmeasurable: Urban design qualities related to walkability. Journal of Urban Design, 14(1), 65-84. http://dx.doi.org/10.1080/13574800802451155

Federal Highway Administration. (2011). 2009 National household travel survey: User's guide. Washington, DC: U.S. Department of Transportation. Retrieved from http://nhts.ornl.gov/2009/pub/UsersGuideV2.pdf

Ferrell, C. E., Mathur, S., \& Mendoza, E. (2008). Neighborhood Crime and Travel Behavior: An Investigation of the Influence of Neighborhood Crime Rates on Mode Choice (MTI Report 07-02). San José, CA: San José State University. Retrieved from http://transweb.sjsu.edu/MTIportal/research/publications/summary/0702.html

Festinger, L. (1957). A theory of cognitive dissonance. Evanston, IL: Row \& Peterson.

Finch, C., Owen, N., \& Price, R. (2001). Current injury or disability as a barrier to being more physically active. Medicine and Science in Sports and Exercise, 33(5), 778782. http://dx.doi.org/10.1097\%2F00005768-200105000-00016

Fiorenzo-Catalano, M. S. (2007). Choice set generation in multi-modal transportation networks. (Unpublished doctoral dissertation). Delft University of Technology, 
Delft, The Netherlands. Retrieved from http://resolver.tudelft.n1/uuid:ef3b9c22b979-4f46-9b02-110c82d67535

Fishbein, M. (1980). A theory of reasoned action: Some applications and implications. Nebraska Symposium on Motivation, 1979, 27, 64-116.

Fishbein, M., \& Ajzan, I. (1975). Belief, attitude, intention and behavior: An introduction to theory and research. Reading, MA: Addison-Wesley Publishing Company.

Flynn, B. S., Dana, G. S., Sears, J., \& Aultman-Hall, L. (2012). Weather factor impacts on commuting to work by bicycle. Preventive Medicine, 54(2), 122-124. http://dx.doi.org/10.1016/j.ypmed.2011.11.002

Foerster, J. F. (1979). Mode choice decision process models: a comparison of compensatory and non-compensatory structures. Transportation Research Part A: General, 13(1), 17-28. http://dx.doi.org/10.1016/0191-2607(79)90083-9

Forsyth, A., \& Krizek, K. (2011). Urban design: Is there a distinctive view from the bicycle? Journal of Urban Design, 16(4), 531-549. http://dx.doi.org/10.1080/13574809.2011.586239

Forsyth, A., Oakes, J. M., Lee, B., \& Schmitz, K. H. (2009). The built environment, walking, and physical activity: Is the environment more important to some people than others? Transportation Research Part D: Transport and Environment, 14(1), 42-49. http://dx.doi.org/10.1016/j.trd.2008.10.003

Fosgerau, M., \& Hess, S. (2009). A comparison of methods for representing random taste heterogeneity in discrete choice models. European Transport, 42, 1-25. Retrieved from http://www.stephanehess.me.uk/papers/Fosgerau_Hess_2008.pdf 
Fosgerau, M., \& Karlström, A. (2010). The value of reliability. Transportation Research Part B: Methodological, 44(1), 38-49. http://dx.doi.org/10.1016/j.trb.2009.05.002

Foster, S., Giles-Corti, B., \& Knuiman, M. (2011). Neighbourhood design and fear of crime: A social-ecological examination of the correlates of residents' fear in new suburban housing developments. Health \& Place, 16(6), 1156-1165. http://dx.doi.org/10.1016/j.jenvp.2010.03.005

Frank, L. D., \& Engelke, P. (2001). How land use and transportation systems impact public health: A literature review of the relationship between physical activity and built form (Working Paper \#1). Atlanta, GA: Active Community Environments Initiative, Centers for Disease Control and Prevention. Retrieved from http://www.cdc.gov/nccdphp/dnpa/pdf/aces-workingpaper1.pdf

Freedman, J., \& Goulias, K. (2012). Activity-based modeling: Webinar session 6: Accessibilities and treatment of space. Presented to the Travel Model Improvement Program, Washington, DC. Retrieved from http://tmiponline.org/Clearinghouse/Items/20120517_-_ActivityBased_Modeling_Accessibility_and_Treatment_of_Space_Session_6.aspx

Frejinger, E., Bierlaire, M., \& Ben-Akiva, M. (2009). Sampling of alternatives for route choice modeling. Transportation Research Part B: Methodological, 43(10), 984994. http://dx.doi.org/10.1016/j.trb.2009.03.001

Frey, B. S. (1988). Ipastive and objective limits to human behavior. The Journal of Behavioral Economics, 17(4), 229-248. 
Fujii, S., \& Kitamura, R. (2003). What does a one-month free bus ticket do to habitual drivers? An experimental analysis of habit and attitude change. Transportation, 30(1), 81-95. http://dx.doi.org/10.1023/A:1021234607980

Galdames, C., Tudela, A., \& Carrasco, J. A. (2011). Exploring the role of psychological factors in mode choice models by a latent variables approach. Transportation Research Record: Journal of the Transportation Research Board, 2230, 68-74. http://dx.doi.org/10.3141/2230-08

Gardner, B. (2009). Modelling motivation and habit in stable travel mode contexts. Transportation Research Part F: Traffic Psychology and Behaviour, 12(1), 6876. http://dx.doi.org/10.1016/j.trf.2008.08.001

Gardner, B., \& Abraham, C. (2007). What drives car use? A grounded theory analysis of commuters' reasons for driving. Transportation Research Part F: Traffic Psychology and Behaviour, 10(3), 187-200. http://dx.doi.org/10.1016/j.trf.2006.09.004

Gärling, T., Gillholm, R., \& Gärling, A. (1998). Reintroducing attitude theory in travel behavior research: The validity of an interactive interview procedure to predict car use. $\quad$ Transportation, $25(2), \quad 129-146$. http://dx.doi.org/10.1023/A:1005004311776

Gärling, T., Kwan, M. P., \& Golledge, R. G. (1994). Computational-process modelling of household activity scheduling. Transportation Research Part B: Methodological, 28(5), 355-364. http://dx.doi.org/10.1016/0191-2615(94)90034-5 
Garvill, J., Marell, A., \& Nordlund, A. (2003). Effects of increased awareness on choice of travel mode. Transportation, $30(1), \quad 63-79$. http://dx.doi.org/10.1023/A:1021286608889

Gehrke, S. R. (2012). An exploration into the influence of geographic scale and land use mix on nonmotorized travel. Presented at the $53^{\text {rd }}$ Annual Conference of the Associatoin of Collegiate Schools of Planning, Cincinnati, OH.

Geller, R. (2006). Four types of cyclists. Retrieved from http://www.portlandoregon.gov/transportation/article/264746

Gilbride, T. J., \& Allenby, G. M. (2004). A choice model with conjunctive, disjunctive, and compensatory screening rules. Marketing Science, 23(3), 391-406. http://dx.doi.org/10.1287/mksc.1030.0032

Giuliano, G., \& Dargay, J. (2006). Car ownership, travel and land use: A comparison of the US and Great Britain. Transportation Research Part A: Policy and Practice, 40(2), 106-124. http://dx.doi.org/10.1016/j.tra.2005.03.002

Gliebe, J., \& Kim, K. (2010). Time-dependent utility in activity and travel choice behavior. Transportation Research Record: Journal of the Transportation Research Board, 2156, 9-16. http://dx.doi.org/10.3141/2156-02

Gliebe, J. P., \& Koppelman, F. S. (2002). A model of joint activity participation between $\begin{array}{llll}\text { household } & \text { members. } & \text { Transportation, } & \text { 29(1), }\end{array}$ http://dx.doi.org/10.1023/A:1012995509777

Gliebe, J. P., \& Koppelman, F. S. (2005). Modeling household activity-travel interactions as parallel constrained choices. Transportation, 32(5), 449-471. http://dx.doi.org/10.1007/s11116-005-5328-0 
Golledge, R. G., \& Gärling, T. (2004). Cognitive maps and urban travel. In D. A. Hensher, K. J. Button, K. E. Haynes, \& P. R. Stopher (Eds.), Handbook of Transport Geography and Spatial Systems (pp. 501-512). Oxford, UK: Elsevier.

Greene, W. H., \& Hensher, D. A. (2003). A latent class model for discrete choice analysis: Contrasts with mixed logit. Transportation Research Part B: Methodological, $\quad 37(8), \quad$ 681-698. $\quad$ http://dx.doi.org/10.1016/S01912615(02)00046-2

Greenwald, M. J., \& Boarnet, M. G. (2001). Built environment as determinant of walking behavior: Analyzing nonwork pedestrian travel in Portland, Oregon. Transportation Research Record: Journal of the Transportation Research Board, 1780, 33-41. http://dx.doi.org/10.3141/1780-05

Griswold, J. B., Medury, A., \& Schneider, R. J. (2011). Pilot models for estimating bicycle intersection volumes. Transportation Research Record: Journal of the Transportation Research Board, 2247, 1-7. http://dx.doi.org/10.3141/2247-01

Guo, Z. (2011). Mind the map! The impact of transit maps on path choice in public transit. Transportation Research Part A: Policy and Practice, 45(7), 625-639. http://dx.doi.org/10.1016/j.tra.2011.04.001

Hägerstrand, T. (1970). What about people in regional science? Papers in Regional Science, 24(1), 6-21. http://dx.doi.org/10.1007/BF01936872

Handy, S. L. (1996). Urban form and pedestrian choices: Study of Austin neighborhoods. Transportation Research Record: Journal of the Transportation Research Board, 1552, 135-144. http://dx.doi.org/10.3141/1552-19 
Handy, S. (2005). Critical assessment of the literature on the relationships among transportation, land use, and physical activity. Does the Built Environment Influence Physical Activity? Examining the Evidence (TRB Special Report 282). Washington, DC: Transportation Research Board. Retrieved from http://onlinepubs.trb.org/onlinepubs/archive/downloads/sr282papers/sr282handy. pdf

Handy, S. (2008). Regional transportation planning in the US: An examination of changes in technical aspects of the planning process in response to changing

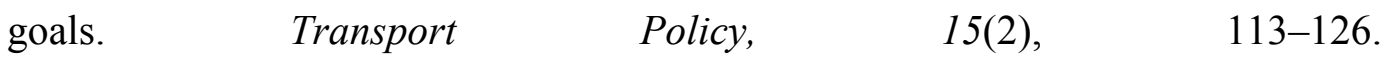
http://dx.doi.org/10.1016/j.tranpol.2007.10.006

Handy, S., Cao, X., \& Mokhtarian, P. (2005). Correlation or causality between the built environment and travel behavior? Evidence from Northern California. Transportation Research Part D: Transport and Environment, 10(6), 427-444. http://dx.doi.org/10.1016/j.trd.2005.05.002

Handy, S., Cao, X., \& Mokhtarian, P. L. (2006). Self-selection in the relationship between the built environment and walking: Empirical evidence from Northern California. Journal of the American Planning Association, 72(1), 55-74. http://dx.doi.org/10.1080/01944360608976724

Handy, S. L., Xing, Y., \& Buehler, T. J. (2010). Factors associated with bicycle ownership and use: A study of six small US cities. Transportation, 37(6), 967985. http://dx.doi.org/10.1007/s11116-010-9269-x 
Hanson, S., \& Hanson, P. (1981). The travel-activity patterns of urban residents: Dimensions and relationships to sociodemographic characteristics. Economic Geography, 57(4), 332-347. http://dx.doi.org/10.2307/144213

Herzog, T. R. (1992). A cognitive analysis of preference for urban spaces. Journal of Environmental Psychology, 12(3), 237-248. http://dx.doi.org/10.1016/S02724944(05)80138-0

Herzog, T., \& Smith, G. A. (1988). Danger, mystery, and environmental preference. Environment and Behavior, 20(3), 320-344. http://dx.doi.org/10.1177/0013916588203004

Hess, P. M., Moudon, A. V., Snyder, M. C., \& Stanilov, K. (1999). Site design and pedestrian travel. Transportation Research Record: Journal of the Transportation Research Board, 1674, 9-19. http://dx.doi.org/10.3141/1674-02

Hess, S., Bierlaire, M. \& Polak, J. W. (2007). A systematic comparison of continuous and discrete mixture models, European Transport, 37, 35-61. Retrieved from http://www.stephanehess.me.uk/papers/hbp_et_final.pdf

Hess, S., Stathopoulos, A., \& Daly, A. (2012). Allowing for heterogeneous decision rules in discrete choice models: An approach and four case studies. Transportation, 39(3), 565-591. http://dx.doi.org/10.1007/s11116-011-9365-6

Hess, S., Stathopoulos, A., Campbell, D., O’Neill, V., \& Caussade, S. (2013). It's not that I don't care, I just don't care very much: Confounding between attribute nonattendance and taste heterogeneity. Transportation, 40(3), 1-25. http://dx.doi.org/10.1007/s1 1116-012-9438-1 
Hess, S., \& Train, K. E. (2011). Recovery of inter-and intra-personal heterogeneity using mixed logit models. Transportation Research Part B: Methodological, 45(7), 973-990. http://dx.doi.org/10.1016/j.trb.2011.05.002

Hill, M. R. (1982). Spatial structure and decision-making of pedestrian route selection through an urban environment. (Unpublished doctoral dissertation). University of Nebraska, Lincoln, NE. $\quad$ Retrieved from http://digitalcommons.unl.edu/dissertations/AAI8306484/

Hollander, Y. (2006). Direct versus indirect models for the effects of unreliability. Transportation Research Part A: Policy and Practice, 40(9), 699-711. http://dx.doi.org/10.1016/j.tra.2005.12.007

Hood, J., Sall, E., \& Charlton, B. (2011). A GPS-based bicycle route choice model for San Francisco, California. Transportation Letters: The International Journal of Transportation Research, $\quad 3(1), \quad$ 63-75. http://dx.doi.org/10.3328/TL.2011.03.01.63-75

Hoogendoorn, S. P., \& Bovy, P. H. (2004). Pedestrian route-choice and activity scheduling theory and models. Transportation Research Part B: Methodological, 38(2), 169-190. http://dx.doi.org/10.1016/S0191-2615(03)00007-9

Hoogendoorn-Lanser, S., Bovy, P., \& van Nes, R. (2007). Application of constrained enumeration approach to multimodal choice set generation. Transportation Research Record: Journal of the Transportation Research Board, 2014, 50-57. http://dx.doi.org/10.3141/2014-07 
Horning, J., El-Geneidy, A., \& Krizek, K. J. (2008). Perceptions of walking distance to neighborhood retail and other public services. Presented at the 87th Annual Meeting of the Transportation Research Board, Washington, DC.

Horowitz, J. L., Koppelman, F. S., \& Lerman, S. R. (1986). A self-instructing course in disaggregate mode choice modeling: Final report (No. UMTA-IA-11-0006-861). Washington, DC: U.S. Department of Transportation. Retrieved from http://ntl.bts.gov/DOCS/381SIC.html

Humpel, N., Owen, N., Iverson, D., Leslie, E., \& Bauman, A. (2004). Perceived environment attributes, residential location, and walking for particular purposes. American Journal of Preventive Medicine, 26(2), 119-125. http://dx.doi.org/10.1016/j.amepre.2003.10.005

Humpel, N., Owen, N., \& Leslie, E. (2002). Environmental factors associated with adults' participation in physical activity: A review. American Journal of Preventive Medicine, 22(3), 188-199. http://dx.doi.org/10.1016/S0749$3797(01) 00426-3$

Humpel, N., Owen, N., Leslie, E., Marshall, A. L., Bauman, A. E., \& Sallis, J. F. (2004). Associations of location and perceived environmental attributes with walking in neighborhoods. American Journal of Health Promotion, 18(3), 239-242. http://dx.doi.org/10.4278/0890-1171-18.3.239

Hutchison, A. J., Breckon, J. D., \& Johnston, L. H. (2009). Physical activity behavior change interventions based on the transtheoretical model: A systematic review. Health Education \& Behavior, 36(5), 829-845. http://dx.doi.org/10.1177/1090198108318491 
Iacono, M., Krizek, K. J., \& El-Geneidy, A. (2010). Measuring non-motorized accessibility: Issues, alternatives, and execution. Journal of Transport Geography, 18(1), 133-140. http://dx.doi.org/10.1016/j.jtrangeo.2009.02.002

Jacobsen, P. L. (2003). Safety in numbers: More walkers and bicyclists, safer walking and bicycling. Injury Prevention, 9(3), 205-209. http://dx.doi.org/10.1136/ip.9.3.205

Janis, I. L., \& Mann, L. (1977). Decision making: A psychological analysis of conflict, choice, and commitment. New York, NY: The Free Press.

Jariyasunant, J., Abou-Zeid, M., Carrel, A., Ekambaram, V., Gaker, D. J., Sengupta, R., \& Walker, J. L. (2013) Quantified traveler: Travel feedback meets the cloud to change behavior. Journal of Intelligent Transportation Systems: Technology, Planning, and Operations. Advance online publication. http://dx.doi.org/10.1080/15472450.2013.856714

Johnson Gardner. (2007). An assessment of the marginal impact of urban amenities on residential pricing. Portland, OR: Metro. Retrived from http://johnsongardner.com/files/Urban_Amenities_Final5.pdf

Jovicic, G. (2001). Activity based travel demand modeling: A literature study. Kongens Lyngby, DK: Danmarks TransportForskning. Retrieved from http://wwwdtu2.sitecore.dtu.dk/upload/institutter/dtu\%20transport/pdf_dtf/notater /not0801.pdf

Kenrick, D. T., Griskevicius, V., Neuberg, S. L., \& Schaller, M. (2010). Renovating the pyramid of needs: Contemporary extensions built upon ancient foundations. 
Perspectives on Psychological Science, 5(3), 292-314. http://dx.doi.org/10.1177/1745691610369469

Khattak, A. J., \& Rodriguez, D. (2005). Travel behavior in neo-traditional neighborhood developments: A case study in USA. Transportation Research Part A: Policy and Practice, 39(6), 481-500. http://dx.doi.org/10.1016/j.tra.2005.02.009

Kim, S., \& Ulfarsson, G. F. (2008). Curbing automobile use for sustainable transportation: analysis of mode choice on short home-based trips. Transportation, 35(6), 723-737. http://dx.doi.org/10.1007/s11116-008-9177-5

Kim, S., Ulfarsson, G. F., \& Hennessy, J. T. (2007). Analysis of light rail rider travel behavior: Impacts of individual, built environment, and crime characteristics on transit access. Transportation Research Part A: Policy and Practice, 41(6), 511522. http://dx.doi.org/10.1016/j.tra.2006.11.001

King, A. C., Castro, C., Wilcox, S., Eyler, A. A., Sallis, J. F., \& Brownson, R. B. (2000). Personal and environmental factors associated with physical inactivity among different racial-ethinic groups of U.S. middle-aged and older-aged women. Health Psychology, 19(4), 354-364. http://dx.doi.org/10.1037/0278-6133.19.4.354

Kingham, S., \& Ussher, S. (2007). An assessment of the benefits of the walking school bus in Christchurch, New Zealand. Transportation Research Part A: Policy and Practice, 41(6), 502-510. http://dx.doi.org/10.1016/j.tra.2006.11.008

Kitamura, R., Mokhtarian, P. L., \& Laidet, L. (1997). A micro-analysis of land use and travel in five neighborhoods in the San Francisco Bay Area. Transportation, 24(2), 125-158. http://dx.doi.org/10.1023/A:1017959825565 
Klöckner, C. A., \& Blöbaum, A. (2010). A comprehensive action determination model: Toward a broader understanding of ecological behaviour using the example of travel mode choice. Journal of Environmental Psychology, 30(4), 574-586. http://dx.doi.org/10.1016/j.jenvp.2010.03.001

Klöckner, C. A., \& Friedrichsmeier, T. (2011). A multi-level approach to travel mode choice: How person characteristics and situation specific aspects determine car use in a student sample. Transportation Research Part F: Traffic Psychology and Behaviour, 14(4), 261-277. http://dx.doi.org/10.1016/j.trf.2011.01.006

Klöckner, C. A., \& Matthies, E. (2004). How habits interfere with norm-directed behaviour: A normative decision-making model for travel mode choice. Journal of Environmental Psychology, 24(3), 319-327. http://dx.doi.org/10.1016/j.jenvp.2004.08.004

Klöckner, C. A., Matthies, E., \& Hunecke, M. (2003). Problems of operationalizing habits and integrating habits in normative decision-making models. Journal of Applied Social Psychology, 33(2), 396-417. http://dx.doi.org/10.1111/j.15591816.2003.tb01902.x

Koppelman, F. S., \& Bhat, C. (2006). A self instructing course in mode choice modeling: multinomial and nested logit models. Washington, DC: U.S. Department of Transportation. Retrieved from http://www.ce.utexas.edu/prof/bhat/COURSES/LM_Draft_060131Final060630.pdf 
Kuppam, A. R., \& Pendyala, R. M. (2001). A structural equations analysis of commuters' activity and travel patterns. Transportation, 28(1), 33-54. http://dx.doi.org/10.1023/A:1005253813277

Kwan, M.-P., \& Lee, J. (2003). Geovisualization of human activity patterns using 3D GIS: A time-geographic approach. In M. F. Goodchild \& D. G. Janelle (Eds.), Spatially integrated social science: Examples in best practice. Oxford, UK: Oxford University Press.

Lam, T. C., \& Small, K. A. (2001). The value of time and reliability: Measurement from a value pricing experiment. Transportation Research Part E: Logistics and Transportation Review, 37(2), 231-251. http://dx.doi.org/10.1016/S1366$5545(00) 00016-8$

Langlois, J. A., Keyl, P. M., Guralnik, J. M., Foley, D. J., Marottoli, R. A., \& Wallace, R. B. (1997). Characteristics of older pedestrians who have difficulty crossing the street. American Journal of Public Health, 87(3), 393-397. http://dx.doi.org/10.2105/AJPH.87.3.393

Lee, C., \& Moudon, A. V. (2004). Physical activity and environment research in the health field: Implications for urban and transportation planning practice and research. Journal of Planning Literature, 19(2), 147-181. http://dx.doi.org/10.1177/0885412204267680

Lerman, S. R. (1976) Location, housing, automobile ownership and mode to work: A joint choice model. Transportation Research Record, 610, 6-11.

Li, Z., Hensher, D. A., \& Rose, J. M. (2010). Willingness to pay for travel time reliability in passenger transport: A review and some new empirical evidence. 
Transportation Research Part E: Logistics and Transportation Review, 46(3), 384-403. http://dx.doi.org/10.1016/j.tre.2009.12.005

Lindsey, G., Chen, J., \& Hankey, S. (2013). Adjustment factors for estimating miles traveled by nonmotorized traffic. Presented at the 92nd Annual Meeting of the Transportation Research Board, Washington, DC.

Liu, F., J. E. Evans, \& T. Rossi. (2012) Recent practices in regional modeling of nonmotorized travel. Transportation Research Record: Journal of the Transportation Research Board, 2303, 1-8. http://dx.doi.org/10.3141/2303-01

Livi Smith, A. (2009). Contribution of perceptions in analysis of walking behavior. Transportation Research Record: Journal of the Transportation Research Board, 2140, 128-136. http://dx.doi.org/10.3141/2140-14

Loukaitou-Sideris, A. (2006). Is it safe to walk? Neighborhood safety and security considerations and their effects on walking. Journal of Planning Literature, 20(3), 219-232. http://dx.doi.org/10.1177/0885412205282770

Loukaitou-Sideris, A., \& Eck, J. E. (2007). Crime prevention and active living. American Journal of Health Promotion, 21(4s), 380-389. http://dx.doi.org/10.4278/0890$1171-21.4 s .380$

Lu, X., \& Pas, E. I. (1999). Socio-demographics, activity participation and travel behavior. Transportation Research Part A: Policy and Practice, 33(1), 1-18. http://dx.doi.org/10.1016/S0965-8564(98)00020-2

Luce, R. D. (1959). Individual choice behavior. New York, NY: Wiley. 
Luce, R. D. (1977). The choice axiom after twenty years. Journal of Mathematical $\begin{array}{llll}\text { Psychology, } & \text { 15(3), } & \text { 215-233. } & \text { Retrieved }\end{array}$ http://www.socsci.uci.edu/ rdluce/bio/pre1990/1977/Luce_JMP_1977a.pdf

Luce, R. D., \& Suppes, P. (1965). Preference, utility, and subjective probability. In R. D. Luce, R. R. Bush, \& E. Galanter (Eds.), Handbook of Mathematical Psychology, Vol. 3 (pp. 249-410). New York, NY: Wiley.

Lynch, K. (1960). The image of the city. Cambridge, MA: The MIT Press.

Ma, J., \& Goulias, K. G. (1997). A dynamic analysis of person and household activity and travel patterns using data from the first two waves in the Puget Sound Transportation Panel. Transportation, 24(3), 309-331. http://dx.doi.org/10.1023/A:1004955507098

Manaugh, K., \& El-Geneidy, A. M. (2013). Does distance matter? Exploring the links among values, motivations, home location, and satisfaction in walking trips. Transportation Research Part A: Policy and Practice, 50, 198-208. http://dx.doi.org/10.1016/j.tra.2013.01.044

Manski, C. F. (1977). The structure of random utility models. Theory and Decision, 8(3), 229-254. http://dx.doi.org/10.1007/BF00133443

Marschak, J. (1960). Binary-choice constraints and random utility indicators. In K. J. Arrow, S. Karlan, \& P. Suppes (Eds.), Mathematical methods in the social sciences, 1959: Proceedings of the first Stanford symposium (pp. 312-329). Stanford, CA: Stanford University Press.

Marshall, S. J., \& Biddle, S. J. (2001). The transtheoretical model of behavior change: A meta-analysis of applications to physical activity and exercise. Annals of 
http://dx.doi.org/10.1207/S15324796ABM2304_2

Maslow, A. H. (1943). A theory of human motivation. Psychological Review, 50(4), 370396. Retrieved from http://psychclassics.yorku.ca/Maslow/motivation.htm

Maslow, A. H. (1954). Motivation and personality. New York, NY: Harper \& Brothers.

Matthies, E., Kuhn, S., \& Klöckner, C. A. (2002). Travel mode choice of women: The result of limitation, ecological norm, or weak habit? Environment and Behavior, 34(2), 163-177. http://dx.doi.org/10.1177/0013916502034002001

McAlister, A. L., Perry, C. L., \& Parcel, G. S. (2008). How individuals, environments, and health behaviors interact: Social cognitive theory. In K. Glanz, B. K. Rimer, \& K. Vizwanath (Eds.), Health behavior and health education: Theory research, and practice $\left(4^{\text {th }}\right.$ ed.) (pp. 169-188). San Francisco, CA: John Wiley \& Sons, Inc.

McConville, M. E., Rodríguez, D. A., Clifton, K., Cho, G., \& Fleischhacker, S. (2011). Disaggregate land uses and walking. American Journal of Preventive Medicine, 40(1), 25-32. http://dx.doi.org/10.1016/j.amepre.2010.09.023

McCormack, G., Giles-Corti, B., Lange, A., Smith, T., Martin, K., \& Pikora, T. J. (2004). An update of recent evidence of the relationship between objective and self-report measures of the physical environment and physical activity behaviours. Journal of Science and Medicine in Sport, 7(1), 81-92. http://dx.doi.org/10.1016/S1440$2440(04) 80282-2$

McDonald, N. C. (2008). Household interactions and children's school travel: The effect of parental work patterns on walking and biking to school. Journal of Transport Geography, 16(5), 324-331. http://dx.doi.org/10.1016/j.jtrangeo.2008.01.002 
McFadden, D. (1973). Conditional logit analysis of qualitative choice behavior. In P. Zarakumba (Ed.), Frontiers in econometrics (pp. 105-142). New York, NY: Academic Press.

McFadden, D. (1974). The measurement of urban travel demand. Journal of Public $\begin{array}{llll}\text { Economics, } & 3(4), & \text { 303-328. } & \text { Retrieved }\end{array}$ http://elsa.berkeley.edu/pub/reprints/mcfadden/measurement.pdf

McFadden, D. (2001). Dissaggregate behavioral travel demand's RUM side: A 30-year retrospective. In D. Heshner (Ed.), Travel behavior research: The leading edge. Oxford: Pergamon Press.

McFadden, D. (2007). The behavioral science of transportation. Transport Policy, 14(4), 269-274. http://dx.doi.org/10.1016/j.tranpol.2007.07.001

McGinn, A. P., Evenson, K. R., Herring, A. H., \& Huston, S. L. (2007). The relationship between leisure, walking, and transportation activity with the natural environment. Health \& Place, 13(3), 588-602. http://dx.doi.org/10.1016/j.healthplace.2006.07.002

McNally, M. G., \& Rindt, C. R. (2008). The activity-based approach. In D. A. Hensher \& K. J. Button (Eds.), Handbook of transport modelling ( $2^{\text {nd }}$ ed.). Bingley, UK: Emerald, Inc.

Metropolitan Washington Council of Governments. (2012). Household travel survey: Personal one-day travel diary. Washington, DC: Metropolitan Washington $\begin{array}{llll}\text { Council of } & \text { Governments. } & \text { Retrieved }\end{array}$ http://www.mwcog.org/transportation/activities/hts/documents/Travel\%20_Diary Final.pdf 
Miller, E. J., Roorda, M. J., \& Carrasco, J. A. (2005). A tour-based model of travel mode choice. Transportation, 32(4), 399-422. http://dx.doi.org/10.1007/s11116-004$7962-3$

Millonig, A., \& Schechtner, K. (2007). Decision loads and route qualities for pedestrians: Key requirements for the design of pedestrian navigation services. Pedestrian and Evacuation Dynamics 2005, 109-118. http://dx.doi.org/10.1007/978-3-54047064-9_10

Mokhtarian, P. L., \& Cao, X. (2008). Examining the impacts of residential self-selection on travel behavior: A focus on methodologies. Transportation Research Part B: Methodological, 42(3), 204-228. http://dx.doi.org/10.1016/j.trb.2007.07.006

Mokhtarian, P. L., \& Salomon, I. (2001). How derived is the demand for travel? Some conceptual and measurement considerations. Transportation Research Part A: Policy and Practice, 35(8), 695-719. http://dx.doi.org/10.1016/S09658564(00)00013-6

Montaño, D. E., \& Kasprzyk, D. (2008). Theory of reasoned action, theory of planned behavior, and the integrated behavioral model. In K. Glanz, B. K. Rimer, \& K. Vizwanath (Eds.), Health behavior and health education: Theory research, and practice ( $4^{\text {th }}$ ed.) (pp. 67-96). San Francisco, CA: John Wiley \& Sons, Inc.

Morgan-Lopez, A. A., \& MacKinnon, D. P. (2006). Demonstration and evaluation of a method for assessing mediated moderation. Behavior Research Methods, 38(1), 77-87. http://dx.doi.org/10.3758/BF03192752 
Muller, D., Judd, C. M., \& Yzerbyt, V. Y. (2005). When moderation is mediated and mediation is moderated. Journal of Personality and Social Psychology, 89(6), 852-863. http://dx.doi.org/10.1037/0022-3514.89.6.852

Nasar, J. L. (1983). Adult viewers' preferences in residential scenes: A study of the relationship of environmental attributes to preference. Environment and Behavior, 15(5), 589-614. http://dx.doi.org/10.1177/0013916583155003

Noland, R. B., \& Polak, J. W. (2002). Travel time variability: A review of theoretical and empirical issues. Transport Reviews, 22(1), 39-54. http://dx.doi.org/10.1080/01441640010022456

Nordback, K., Marshall, W., Janson, B. N., \& Stolz, E. (2013). Estimating annual average daily bicyclists: Error and accuracy. Presented at the 92nd Annual Meeting of the Transportation Research Board, Washington, DC.

Nourzad, F. (2000) Modeling of the non-motorized travel interregional travel model development (Phase I-Task 3) (Memo). Salt Lake City, UT: Wasatch Front Regional Council.

Oakes, J. M., Forsyth, A., \& Schmitz, K. H. (2007). The effects of neighborhood density and street connectivity on walking behavior: the Twin Cities walking study. Epidemiologic Perspectives \& Innovations, $\quad 4(1), \quad 16$. http://dx.doi.org/10.1186/1742-5573-4-16

Oregon Modeling Steering Committee. (2011). Oregon travel and activity survey, 20092011. Retrieved from http://www.oregon.gov/ODOT/TD/TP/pages/travelsurvey.aspx/ 
Oregon Modeling Steering Committee. (2012). Oregon travel and activity survey: Personal one-day travel diary. Salem, OR: Oregon Modeling Steering Committee. Retrieved from http://www.oregon.gov/ODOT/TD/TP/docs/travelsurvey/diary.pdf

Ortúzar, J. d. D., \& Willumsen, L. G. (2011). Modelling transport. Chichister, UK: John Wiley \& Sons, Ltd.

Ory, D. T., \& Mokhtarian, P. L. (2005). When is getting there half the fun? Modeling the liking for travel. Transportation Research Part A: Policy and Practice, 39(2), 97123. http://dx.doi.org/10.1016/j.tra.2004.09.006

Outwater, M. L., Spitz, G., Lobb, J., Campbell, M., Sana, B., Pendyala, R., \& Woodford, W. Characteristics of premium transit service that affect mode choice: TCRP H37 summary of phase 1. Transportation, 38(4), 605-623. http://dx.doi.org/10.1007/s11116-011-9334-0

Owen, N., Humpel, N., Leslie, E., Bauman, A., \& Sallis, J. F. (2004). Understanding environmental influences on walking: Review and research agenda. American Journal of Preventive Medicine, 27(1), 67-76. http://dx.doi.org/10.1016/j.amepre.2004.03.006

Oyeyemi, A. L., Adegoke, B. O., Sallis, J. F., Oyeyemi, A. Y., \& De Bourdeaudhuij, I. (2012). Perceived crime and traffic safety is related to physical activity among adults in Nigeria. BMC Public Health, 12(1), 294. http://dx.doi.org/10.1186/1471$2458-12-294$

Paez, A., Scott, D., Potoglou, D., Kanaroglou, P., \& Newbold, K. B. (2007). Elderly mobility: Demographic and spatial analysis of trip making in the Hamilton CMA, 
$\begin{array}{llll}\text { Canada. } & \text { Urban } & \text { Studies, } & \text { 44(1), }\end{array}$ http://dx.doi.org/10.1080/00420980601023885

Parra, D. C., Hoehner, C. M., Hallal, P. C., Ribeiro, I. C., Reis, R., Brownson, R. C., ... Simoes, E. J. (2011). Perceived environmental correlates of physical activity for leisure and transportation in Curitiba, Brazil. Preventive Medicine, 52(3), 234238. http://dx.doi.org/10.1016/j.ypmed.2010.12.008

Parsons Brinkerhoff. (2012). Strategic Highway Research Program (SHRP) II: Project C04: Improving our understanding of how highway congestion and pricing affect travel demand: Phase III: Revised final report: Executive summary and technical report. Washington, DC: Transportation Research Board. Retrieved from http://onlinepubs.trb.org/onlinepubs/shrp2/SHRP2prepubC04.pdf

Parsons Brinckerhoff Quade and Douglas, Inc., Cambridge Systematics, Inc., \& Calthorpe Associates. (1993). Making the land use transportation air quality connection: The pedestrian environment: Volume 4A. Portland, OR: 1000 Friends of Oregon. Retrieved from http://www.friends.org/resources/reports

Pas, E. I. (1985). State of the art and research opportunities in travel demand: Another perspective. Transportation Research Part A: General, 19(5), 460-464. http://dx.doi.org/10.1016/0191-2607(85)90048-2

Pendyala, R. M., \& Pas, E. I. (2000). Multi-day and multi-period data for travel demand analysis and modeling. In Transport surveys: Raising the standard (pp. II-B/128) (Transportation Research E-Circulator Number E-C008). Washington, DC: Transportation Research Board. 
Pikora, T., Giles-Corti, B., Bull, F., Jamrozik, K., \& Donovan, R. (2003). Developing a framework for assessment of the environmental determinants of walking and cycling. Social Science \& Medicine, 56(8), 1693-1703. http://dx.doi.org/10.1016/S0277-9536(02)00163-6

Pinjari, A. R., Pendyala, R. M., Bhat, C. R., \& Waddell, P. A. (2011). Modeling the choice continuum: An integrated model of residential location, auto ownership, bicycle ownership, and commute tour mode choice decisions. Transportation, 38(6), 933-958. http://dx.doi.org/10.1007/s11116-011-9360-y

Pizam, A., \& Mansfield, Y. (Eds.). (1999). Consumer Behavior in Travel and Tourism. New York, NY: The Haworth Press, Inc.

Plaut, P. O. (2005). Non-motorized commuting in the US. Transportation Research Part D: Transport and Environment, 10(5), 347-356. http://dx.doi.org/10.1016/j.trd.2005.04.002

Porter, C., Suhrbier, J., \& Schwartz, W. L. (1999). Forecasting bicycle and pedestrian travel: State of the practice and research needs. Transportation Research Record: Journal of the Transportation Research Board, 1674, 94-101. http://dx.doi.org/10.3141/1674-13

Prato, C. G., \& Bekhor, S. (2006). Applying branch-and-bound technique to route choice set generation. Transportation Research Record: Journal of the Transportation Research Board, 1985, 19-28. http://dx.doi.org/10.3141/1985-03

Preacher, K. J., Rucker, D. D., \& Hayes, A. F. (2007). Addressing moderated mediation hypotheses: Theory, methods, and prescriptions. Multivariate Behavioral Research, 42(1), 185-227. http://dx.doi.org/10.1080/00273170701341316 
Prochaska, J. O. (2008). Decision making in the transtheoretical model of behavior change. Medical Decision Making, 28(6), 845-849. http://dx.doi.org/10.1177/0272989X08327068

Prochaska, J. O., Redding, C. A., \& Evers, K. E. (2008). The transtheoretical model and stages of change. In K. Glanz, B. K. Rimer, \& K. Vizwanath (Eds.), Health behavior and health education: Theory research, and practice ( $4^{\text {th }}$ ed.) (pp. 97121). San Francisco, CA: John Wiley \& Sons, Inc.

Prochaska, J. O., \& Velicer, W. F. (1997). The transtheoretical model of health behavior change. American Journal of Health Promotion, 12(1), 38-48. http://dx.doi.org/10.4278/0890-1171-12.1.38

Pucher, J., \& Dijkstra, L. (2003). Promoting safe walking and cycling to improve public health: Lessons from the Netherlands and Germany. American Journal of Public Health, 93(9), 1509-1516. http://dx.doi.org/10.2105/AJPH.93.9.1509

Purvis, C. (1997). Review of the literature: Home-based work mode choice models: Technical memorandum HBWMC \#1 (Memo). Oakland, CA: Metropolitan $\begin{array}{llll}\text { Transportation } & \text { Commission. } & \text { Retrieved } & \text { from }\end{array}$ http://www.mtc.ca.gov/maps_and_data/index.htm

Quarmby, D. A. (1967). Choice of travel mode for the journey to work: some findings. Journal of Transport Economics and Policy, 1(3), 273-314. Retrieved from http://www.jstor.org/stable/20052067

Raford, N., \& Ragland, D. (2004). Space syntax: Innovative pedestrian volume modeling tool for pedestrian safety. Transportation Research Record: Journal of the Transportation Research Board, 1878, 66-74. http://dx.doi.org/10.3141/1878-09 
Ramming, M. S. (2002). Network knowledge and route choice. (Unpublished doctoral dissertation). Massachusetts Institute of Technology, Cambridge, MA. Retrieved from http://dspace.mit.edu/handle/1721.1/49797

Raubel, M., \& Winter, S. (2002). Enriching wayfinding instructions with local landmarks. Geographic Information Science, 2478, 243-259. http://dx.doi.org/10.1007/3-540-45799-2_17

RDC, Inc. (1995). Activity based modeling system for travel demand forecasting. Washington, DC: Travel Model Improvement Program. Retrieved from http://www.nctcog.org/trans/modeling/nextgeneration/ActivityBasedModelingSys tem.pdf

Recker, W. W., \& Golob, T. F. (1979). A non-compensatory model of transportation behavior based on sequential consideration of attributes. Transportation Research Part B: Methodological, 13(4), 269-280. http://dx.doi.org/10.1016/01912615(79)90019-5

Recker, W. W., McNally, M. G., \& Root, G. S. (1986a). A model of complex travel behavior: Part I: Theoretical development. Transportation Research Part A: General, 20(4), 307-318. http://dx.doi.org/10.1016/0191-2607(86)90089-0

Recker, W. W., McNally, M. G., \& Root, G. S. (1986b). A model of complex travel behavior: Part II: An operational model. Transportation Research Part A: General, 20(4), 319-330. http://dx.doi.org/10.1016/0191-2607(86)90090-7

Redmond, L. S., \& Mokhtarian, P. L. (2001). The positive utility of the commute: Modeling ideal commute time and relative desired commute amount. Transportation, 28(2), 179-205. http://dx.doi.org/10.1023/A:1010366321778 
Reibstein, D. J., Lovelock, C. H., \& Dobson, R. d. P. (1980). The direction of causality between perceptions, affect, and behavior: An application to travel behavior. Journal of Consumer Research, 6(4), 370-376. http://dx.doi.org/10.1086/208780

Reiff, B., \& Kim, K.-H. (2003). Statistical analysis of urban design variables and their use in travel demand models. Salem, OR: Oregon Department of Transportation. Retrieved from http://www.oregon.gov/ODOT/TD/TP/docs/reports/urbandesignreport.pdf

Reilly, M., \& Landis, J. (2003). The influence of built-form and land use on mode choice (Paper IURD WP 2002 - 4(1)). Berkeley, CA: University of California Transportation Center. Retrieved from http://www.uctc.net/papers/669.pdf

Replogle, M. (1997). Integrating pedestrian and bicycle factors into regional transportation planning models: Summary of the state-of-the-art and suggested steps forward. Urban design, telecommuting and travel forecasting conference: Summary, recommendations, and compendium of papers (Publication No. DOTT-98-2). Arlington, TX: Texas Transportation Institute. Retrieved from http://media.tmiponline.org/clearinghouse/udes/replogle.htm

Rodríguez, D. A., Aytur, S., Forsyth, A., Oakes, J. M., \& Clifton, K. J. (2008). Relation of modifiable neighborhood attributes to walking. Preventive Medicine, 47(3), 260-264. http://dx.doi.org/10.1016/j.ypmed.2008.02.028

Rodríguez, D. A., \& Joo, J. (2004). The relationship between non-motorized mode choice and the local physical environment. Transportation Research Part D: Transport and Environment, 9(2), 151-173. http://dx.doi.org/10.1016/j.trd.2003.11.001 
Ronis, D. L., Yates, J. F., \& Kirscht, J. P. (1989). Attitudes, decisions, and habits as determinants of repeated behavior. In A. R. Pratkanis, S. J. Breckler, \& A. G. Greenwald (Eds.), Attitude structure and function (pp. 213-239). Hillsdale, NJ: Lawrence Erlbaum Associates, Inc.

Ross, C. E. (2000). Walking, exercising, and smoking: Does neighborhood matter? Social Science \& Medicine, 51(2), 265-274. http://dx.doi.org/10.1016/S02779536(99)00451-7

Rossi, T. F. (2000). Modeling non-motorized travel. Presented at the 79th Annual Meeting of the Transportation Research Board, Washington, DC.

Saelens, B. E., \& Handy, S. L. (2008). Built environment correlates of walking: A review. Medicine and Science in Sports and Sxercise, $40(7$ Suppl), S550-S566. http://dx.doi.org/10.1249/MSS.0b013e31817c67a4

Saelens, B. E., Sallis, J. F., Black, J. B., \& Chen, D. (2003). Neighborhood-based differences in physical activity: An environment scale evaluation. American Journal of Public Health, 93(9), 1552-1558. http://dx.doi.org/10.2105/AJPH.93.9.1552

Saelens, B. E., Sallis, J. F., \& Frank, L. D. (2003). Environmental correlates of walking and cycling: Findings from the transportation, urban design, and planning literatures. Annals of Behavioral Medicine, 25(2), 80-91. http://dx.doi.org/10.1207/S15324796ABM2502_03

Saelens, B. E., Sallis, J. F., Frank, L. D., Cain, K. L., Conway, T. L., Chapman, J. E., ... Kerr, J. (2012). Neighborhood environment and psychosocial correlates of adults' 
physical activity. Medicine and Science in Sports and Exercise, 44(4), 637-646. http://dx.doi.org/10.1249/MSS.0b013e318237fe18

Sallis, J. F., Cervero, R. B., Ascher, W., Henderson, K. A., Kraft, M. K., \& Kerr, J. (2006). An ecological approach to creating active living communities. Annual Review of Public Health, 27, 297-322. http://dx.doi.org/10.1146/annurev.publhealth.27.021405.102100

Sallis, J. F., Owen, N., \& Fischer, E. B. (2008). Ecological models of health behavior. In K. Glanz, B. K. Rimer, \& K. Vizwanath (Eds.), Health behavior and health education: Theory research, and practice $\left(4^{\text {th }}\right.$ ed.) (pp. 465-485). San Francisco, CA: John Wiley \& Sons, Inc.

Salmon, J., Owen, N., Crawford, D., Bauman, A., \& Sallis, J. F. (2003). Physical activity and sedentary behavior: A population-based study of barriers, enjoyment, and preference. Health Psychology, 22(2), 178-188. http://dx.doi.org/10.1037/02786133.22 .2 .178

Salomon, I., \& Salomon, M. (1984). Telecommuting: The employee's perspective. Technological Forecasting and Social Change, 25(1), 15-28. http://dx.doi.org/10.1016/0040-1625(84)90077-5

Schneider, R. J., Arnold, L. S., \& Ragland, D. R. (2009). Methodology for counting pedestrians at intersections. Transportation Research Record: Journal of the Transportation Research Board, 2140, 1-12. http://dx.doi.org/10.3141/2140-02

Schneider, R. J. (2011). Understanding sustainable transportation choice: Shifting routine automobile travel to walking and bicycling. (Unpublished doctoral 
dissertation). University of California, Berkeley. Retrieved form http://www.escholarship.org/uc/item/06v2g6dh

Schneider, R. J. (2013). Theory of routine mode choice decisions: An operational framework to increase sustainable transportation. Transport Policy, 25, 128-137. http://dx.doi.org/10.1016/j.tranpol.2012.10.007

Schwanen, T. (2007). Gender differences in chauffeuring children among dual-earner families. The Professional Geographer, 59(4), 447-462. http://dx.doi.org/10.1111/j.1467-9272.2007.00634.x

Schwanen, T., Ettema, D. F., \& Timmermans, H. (2007). If you pick up the children, I'll do the groceries: Spatial differences in between-partner interactions in out-ofhome household activities. Environment and Planning A, 39(11), 2754-2773. http://dx.doi.org/10.1068/a38491

Schwartz, S. H., \& Howard, J. A. (1981). A normative decision-making model of altriusm. In J. P. Rushton \& R. M. Sorrentino (Eds.), Altruism and helping behavior: Social, personality, and developmental perspectives (pp. 189-211). Hillsdale, NJ: Lawrence Erlbaum Associates, Inc.

Sener, I. N., Eluru, N., \& Bhat, C. R. (2009). An analysis of bicycle route choice preferences in Texas, US. Transportation, 36(5), 511-539. http://dx.doi.org/10.1007/s11116-009-9201-4

Singleton, P. A., \& Clifton, K. J. (2013). Pedestrians in regional travel demand forecasting models: State-of-the-practice. Presented at the 92nd Annual Meeting of the Transportation Research Board, Washington, DC. 
Singleton, P. A., \& Wang, L. M. (2014). Safety and security in discretionary travel decision-making: A focus on active travel mode and destination choice. To be presented at the $93^{\text {rd }}$ Annual Meeting of the Transportation Research Board, Washington, DC.

Smith, O. (2013). Commute well-being among bicycle, transit, and car users in Portland, Oregon. Presented at the 92nd Annual Meeting of the Transportation Research Board, Washington, DC.

Snjiders, T. A. B., \& Bosker, R. J. (2012). Multilevel analysis: An introduction to basic and advanced multilevel modeling ( $2^{\text {nd }}$ ed.). Thousand Oaks, CA: SAGE Publications Inc.

Srinivasan, S., \& Bhat, C. R. (2005). Modeling household interactions in daily in-home and out-of-home maintenance activity participation. Transportation, 32(5), 523544. http://dx.doi.org/10.1007/s11116-005-5329-z

Stamps III, A. E. (1999). Demographic effects in environmental aesthetics: A metaanalysis. Journal of Planning Literature, 14(2), 155-175. http://dx.doi.org/10.1177/08854129922092630

Stamps III, A. E. (2002). Entropy, visual diversity, and preference. The Journal of $\begin{array}{lll}\text { General } & \text { Psychology, } & \text { 129(3), }\end{array}$ http://dx.doi.org/10.1080/00221300209602100

Stamps III, A. E. (2005). Visual permeability, locomotive permeability, safety, and enclosure. Environment and Behavior, 37(5), 587-619. http://dx.doi.org/10.1177/0013916505276741 
Stamps III, A. E., \& Nasar, J. L. (1997). Design review and public preferences: Effects of geographical location, public consensus, sensation seeking, and architectural styles. Journal of Environmental Psychology, 17(1), 11-32. http://dx.doi.org/10.1006/jevp.1996.0036

Steg, L. (2003). Can public transport compete with the private car? IATSS Research, 27(2), 27-35. $\quad$ Retrieved from http://www.rug.nl/staff/e.m.steg/stegcanpublictransport.pdf

Steg, L. (2005). Car use: Lust and must. Instrumental, symbolic and affective motives for car use. Transportation Research Part A: Policy and Practice, 39(2), 147-162. http://dx.doi.org/10.1016/j.tra.2004.07.001

Stein, B. (2011). Regional bicycle demand model: In use today in Portland. Presented at the 13th National Transportation Planning Applications Conference, Reno, NV. Retrieved from http://www.trbappcon.org/2011 conf/program.html\#s9b

Stinson, M. A., \& Bhat, C. R. (2003). Commuter bicyclist route choice: Analysis using a stated preference survey. Transportation Research Record: Journal of the Transportation Research Board, 1828, 107-115. http://dx.doi.org/10.3141/182813

Stopher, P. R. (1992). Use of an activity-based diary to collect household travel data. Transportation, 19(2), 159-176. http://dx.doi.org/10.1007/BF02132836

Stopher, P. (2012). Collecting, managing, and assessing data using sample surveys. New York, NY: Cambridge University Press. 
Stopher, P. R., \& Greaves, S. P. (2007). Household travel surveys: Where are we going? Transportation Research Part A: Policy and Practice, 41(5), 367-381. http://dx.doi.org/10.1016/j.tra.2006.09.005

Svenson, O. (1979). Process descriptions of decision making. Organizational Behavior and Human Performance, 23(1), 86-112. http://dx.doi.org/10.1016/00305073(79)90048-5

Swait, J. (2001). A non-compensatory choice model incorporating attribute cutoffs. Transportation Research Part B: Methodological, 35(10), 903-928. http://dx.doi.org/10.1016/S0191-2615(00)00030-8

Swait, J., \& Ben-Akiva, M. (1987a). Incorporating random constraints in discrete models of choice set generation. Transportation Research Part B: Methodological, 21(2), 91-102. http://dx.doi.org/10.1016/0191-2615(87)90009-9

Swait, J., \& Ben-Akiva, M. (1987b). Empirical test of a constrained choice discrete model: mode choice in Sao Paulo, Brazil. Transportation Research Part B: Methodological, 21(2), 103-115. http://dx.doi.org/10.1016/0191-2615(87)900105

Tal, G., \& Handy, S. (2012). Measuring nonmotorized accessibility and connectivity in a robust pedestrian network. Transportation Research Record: Journal of the Transportation Research Board, 2299, 48-56. http://dx.doi.org/10.3141/2299-06

Tanner, C. (1999). Constraints on environmental behaviour. Journal of Environmental Psychology, 19(2), 145-157. http://dx.doi.org/10.1006/jevp.1999.0121

Taylor, N. (2009). Legibility and aesthetics in urban design. Journal of Urban Design, 14(2), 189-202. http://dx.doi.org/10.1080/13574800802670929 
Tertoolen, G., van Kreveld, D., \& Verstraten, B. (1998). Psychological resistance against attempts to reduce private car use. Transportation Research Part A: Policy and Practice, 32(3), 171-181. http://dx.doi.org/10.1016/S0965-8564(97)00006-2

Thøgersen, J. (2006). Understanding repetitive travel mode choices in a stable context: A panel study approach. Transportation Research Part A: Policy and Practice, 40(8), 621-638. http://dx.doi.org/10.1016/j.tra.2005.11.004

Thurstone, L. L. (1927). A law of comparative judgment. Psychological Review, 34, 273286. http://dx.doi.org/10.1037/h0070288

Timmermans, H. (Ed.). (2005). Progress in activity-based analysis. Bingly, UK: Elsevier Science Ltd.

Timms, P., \& Tight, M. (2010). Aesthetic aspects of walking and cycling. Built Environment, 36(4), 487-503. http://dx.doi.org/10.2148/benv.36.4.487

Timperio, A., Crawford, D., Telford, A., \& Salmon, J. (2004). Perceptions about the local neighborhood and walking and cycling among children. Preventive Medicine, 38(1), 39-47. http://dx.doi.org/10.1016/j.ypmed.2003.09.026

Tom, A., \& Denis, M. (2003). Referring to landmark or street information in route directions: What difference does it make? Spatial Information Theory. Foundations of Geographic Information Science, 2825, 362-374. http://dx.doi.org/10.1007/978-3-540-39923-0_24

Train, K. (1980). A structured logit model of auto ownership and mode choice. The Review of Economic Studies, 47(2), 357-370. http://dx.doi.org/10.2307/2296997

Train, K. (2009). Discrete choice methods with simulation. New York, NY: Cambridge University Press. 
Transportation Planning Capacity Building Program. (n.d.) Metropolitan Planning Organization (MPO) Database. Washington, DC: U.S. Department of Transportation. Retrieved from http://www.planning.dot.gov/mpo.asp

Trapp, G. S., Giles-Corti, B., Christian, H. E., Bulsara, M., Timperio, A. F., McCormack, G. R., \& Villaneuva, K. P. (2012). Increasing children's physical activity: Individual, social, and environmental factors associated with walking to and from school. Health Education \& Behavior, 39(2), 172-182. http://dx.doi.org/10.1177/1090198111423272

Triandis, H. C. (1977). Interpersonal behavior. Monterey, CA: Brooks/Cole Publishing Company.

Triandis, H. C. (1980) Values, attitudes, and interpersonal behavior. Nebraska Symposium on Motivation, 1979, 27, 195-259.

Troped, P. J., Saunders, R. P., Pate, R. R., Reininger, B., Ureda, J. R., \& Thompson, S. J. (2001). Associations between self-reported and objective physical environmental factors and use of a community rail-trail. Preventive Medicine, 32(2), 191-200. http://dx.doi.org/10.1006/pmed.2000.0788

Tucker, P., \& Gilliland, J. (2007). The effect of season and weather on physical activity: A systematic review. Public Health, 121(12), 909-922. http://dx.doi.org/10.1016/j.puhe.2007.04.009

Tversky, A. (1972a). Elimination by aspects: A theory of choice. Psychological Review, 79(4), 281-299. http://dx.doi.org/10.1037/h0032955

Tversky, A. (1972b). Choice by elimination. Journal of Mathematical Psychology, 9(4), 341-367. http://dx.doi.org/10.1016/0022-2496(72)90011-9 
US Census Bureau. (2013). TIGER/Line ${ }^{\circledR}$ shapefiles and TIGER/Line ${ }^{\circledR}$ files. Retrieved from https://www.census.gov/geo/maps-data/data/tiger-line.html

Vallerand, R. J. (1997). Toward a hierarchical model of intrinsic and extrinsic motivation. Advances in Experimental Social Psychology, 29, 271-360. http://dx.doi.org/10.1016/S0065-2601(08)60019-2

Van Acker, V., van Wee, B., \& Witlox, F. (2010). When transport geography meets social psychology: Toward a conceptual model of travel behaviour. Transport Reviews, 30(2), 219-240. http://dx.doi.org/10.1080/01441640902943453

Van Acker, V., \& Witlox, F. (2010). Car ownership as a mediating variable in car travel behaviour research using a structural equation modelling approach to identify its dual relationship. Journal of Transport Geography, 18(1), 65-74. http://dx.doi.org/10.1016/j.jtrangeo.2009.05.006

Vanasse Hangen Brustlin, Inc. (2007). Determination of the state of the practice in metropolitan area travel forecasting: Findings of the surveys of metropolitan planning organizations. Washington, DC: Transportation Research Board. Retrieved from http://onlinepubs.trb.org/onlinepubs/reports/vhb-2007-final.pdf van der Waerden, P. J. H. J., Borgers, A., \& Timmermans, H. J. P. (2004). Choice set composition in the context of pedestrians' route choice modeling. Presented at the 83rd Annual Meeting of the Transportation Research Board, Washington, DC.

Van Vugt, M., Van Lange, P. A., Meertens, R. M., \& Joireman, J. A. (1996). How a structural solution to a real-world social dilemma failed: A field experiment on the first carpool lane in Europe. Social Psychology Quarterly, 59(4), 364-374. http://dx.doi.org/10.2307/2787077 
Verlander, N. Q., \& Heydecker, B. G. (1997). Pedestrian route choice: An empirical study. Transportation Planning Methods, 11, 39-49.

Verplanken, B., Aarts, H., van Knippenberg, A., \& van Knippenberg, C. (1994). Attitude versus General Habit: Antecedents of Travel Mode Choice. Journal of Applied Social Psychology, 24(4), 285-300. http://dx.doi.org/10.1111/j.15591816.1994.tb00583.x

Verplanken, B., \& Orbell, S. (2003). Reflections on past behavior: A self-report index of habit strength. Journal of Applied Social Psychology, 33(6), 1313-1330. http://dx.doi.org/10.1111/j.1559-1816.2003.tb01951.x

Verplanken, B., Walker, I., Davis, A., \& Jurasek, M. (2008). Context change and travel mode choice: Combining the habit discontinuity and self-activation hypotheses. Journal of Environmental Psychology, 28(2), 121-127. http://dx.doi.org/10.1016/j.jenvp.2007.10.005

Vovsha, P. (2012). Incorporation of travel time reliability in integrated demand and network simulation models. Presented at the 4th Transportation Research Board Conference on Innovations in Travel Modeling (ITM), Tampa, FL. Retrieved from http://web.anl.gov/TRACC/Training/TMS/04_29_12/index.html

Vovsha, P., Peterson, E., \& Donnelly, R. (2005). Impact of intrahousehold interactions on individual daily activity-travel patterns. Transportation Research Record: Journal of the Transportation Research Board, 1898, 87-97. http://dx.doi.org/10.3141/1898-11

Wall, R., Devine-Wright, P., \& Mill, G. A. (2007). Comparing and combining theories to explain proenvironmental intentions: The case of commuting-mode choice. 
Environment and Behavior, 39(6), 731-753. http://dx.doi.org/10.1177/0013916506294594

Weiner, E. (2013). Urban transportation planning in the United States: History, policy, and practice $\left(4^{\text {th }}\right.$ ed.). New York, NY: Springer.

Wen, C. H., Wang, W. C., \& Fu, C. (2012). Latent class nested logit model for analyzing high-speed rail access mode choice. Transportation Research Part E: Logistics and Transportation $\quad$ Review, $\quad 48(2), \quad 545-554$. http://dx.doi.org/10.1016/j.tre.2011.09.002

Westerdijk, P. K. (1990). Pedestrian and pedal cyclist route choice criteria (ITS Working Paper 302). Haren, The Netherlands: University of Groningen. Retrieved from http://eprints.whiterose.ac.uk/2257/1/ITS155_WP302_uploadable.pdf

Winters, M., Davidson, G., Kao, D., \& Teschke, K. (2011). Motivators and deterrents of bicycling: comparing influences on decisions to ride. Transportation, 38(1), 153168. http://dx.doi.org/10.1007/s11116-010-9284-y

Yarlagadda, A. K., \& Srinivasan, S. (2008). Modeling children's school travel mode and parental escort decisions. Transportation, 35(2), 201-218. http://dx.doi.org/10.1007/s11116-007-9144-6

Young, W. (1986). The role of thresholds in transport choice. Behavioural Research for Transport Policy, 153-170.

Zacharias, J. (2001). Pedestrian behavior pedestrian behavior and perception in urban walking environments. Journal of Planning Literature, 16(1), 3-18. http://dx.doi.org/10.1177/08854120122093249 
Zhou, H., Yang, J., Hsu, P., \& Chen, S. (2010). Factors affecting students' walking/biking rates: Initial findings from a safe route to school survey in Florida. Journal of Transportation Safety \& Security, 2(1), 14-27. http://dx.doi.org/10.1080/19439960903564819

Zorn, L., Sall, E. A., \& Bomberg, M. (2012). Completing the cycle: Incorporating CycleTracks into SF-CHAMP. Presented at the 4th Annual Conference on Innovations in Travel Modeling, Tampa, FL. 